Portland State University

PDXScholar

$1-1-1973$

\title{
A new inquiring system for technology transfer and its role in planning and policymaking
}

Kishandutt Jaydayal Sharma

Portland State University

Follow this and additional works at: https://pdxscholar.library.pdx.edu/open_access_etds Let us know how access to this document benefits you.

\section{Recommended Citation}

Sharma, Kishandutt Jaydayal, "A new inquiring system for technology transfer and its role in planning and policymaking" (1973). Dissertations and Theses. Paper 582.

https://doi.org/10.15760/etd.582

This Dissertation is brought to you for free and open access. It has been accepted for inclusion in Dissertations and Theses by an authorized administrator of PDXScholar. Please contact us if we can make this document more accessible: pdxscholar@pdx.edu. 


\section{A NEW INQUIRING SYSTEM FOR TECHNOLOGY TRANSFER AND ITS ROLE IN PIANNING AND POLICYMAKING}

\section{by}

Kishandutt Jaydayal Sharma

A dissertation submitted in partial fulfillment of the requirements for the degree of

DOCTOR OF PHILOSOPHY

is

SYSTEMS SCIENCE

Portland State University

$1: 73$ 
TO THE OFFICE OF GRADUATE STUDIES AND RESEARCH;

The members of the Committee approve the dissertation of Kishanduti Jaydayal Sharma.

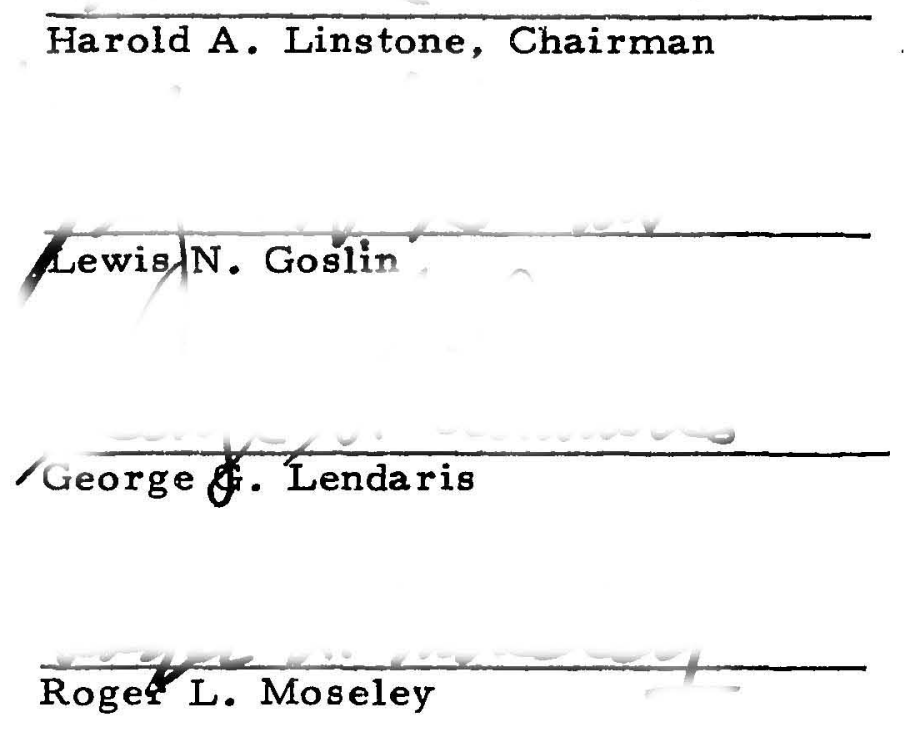

APPROVED:

Harold A, linstone, Director, Systems Science Ph.D. Program David T. Clark, Dean of Graduate Studies and Research 
AN ABSTRACT OF THE THESIS OF Kishandutt $J$. Sharma for the Doctor of Philosophy in Systems Science.

Title: A New Inquiring System for Technology Transfer and Its Role in Planning and Policymaking.

The importance of technology transfer to produce structural change in developing regions has been well established. However, most

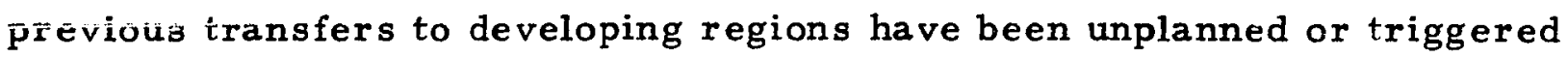
by actior:s rather than decisions. Most previous inquiries have been based on unidimensional. sectorial and mostly econonic views of technology transfer; this author believes these to be inadequate representations of the transfer process.

This dissertation proposes a new inquiring system for technology transfe:; one which emphasizes a multidimensional viewpoint of the technology transfer process. Further, this dissertation claims to make three unique contributions:

1. a new hierarchical structure for examining the technology transfer problem.

2. three measures for assessing the performance of technology transfer. 


\section{ABSTRACT continued}

3. a demonstration example $c_{i} i h \Rightarrow$ suggested methodology. Special emphasis is placed on the needs of planners and policymakers who are considered the clients of the profosed inquiring systems.

The hierarchical structure has three levels, each emphasizing the three critical dimensions of technolos, transfer: 1) resources, 2) transfer mechanisms, and 3) economic and sociocultural consequences. The goals and/or results at each of the three levels of the hierarchical structure are:

Level 1: At this level, a novil soneralized morphological tree is developed to indicate structural differences of technology transfer along the three critical dimensions.

Level 2: At this level, interactions between the variables specified in Level 1 are considered. These interactions are expressed in the form of self-interaction and cross-interactior: natrices. The proposed struccure of technology transfer is established in terms of these matrices.

Level 3: This level specifies, in a flowgraph form, the decision, information and logic requirements to design a "most satisfactory" technology transfer.

The development of these three levels combines quantitative and qualitative viewpoints, since a strictly quantitative approach would lead to an incomplete, unrealistic representation the problem.

The three measures to assess the porformance of technology transfer are: feasibility in terms of rescrices required for transfer, 


\section{ABSTRACT continued}

efficiency expressed in terms of applicability of transfer mechanisms, and desirability in terms of the consequences of transfer. Policy statements, in the form of logic or assignment statements, a re developed to estimate the nature and magnitude of the three performance measures.

A horizontal transfer, specifically the transfer of computer technology to India, is considered to illustrate the proposed methodology. The hierarchical structure is developed and performance measures for the transfer are estimated. The results indicate: (a) the transfer is feasible in terms of most resource requirements, but under the constraints of domestic availability, productivity and cost, (b) the channel -direct investment by business -- is most applicable for the transfer. The channels -- entrepreneur and licensing arrangements -- are of limited applicability. The mechanism -- direct investrnent by Indian government -is applicable if long transfer times can be tolerated, and (c) the economic consequences of the transfer are significant and desirable. However, the transfer is found to have little impact on such important sectors as literacy, food, communication and characters of social organization. 


\section{PREFACE}

In proposing a new and practical approach to a difficult and complex problem, a normal reaction is "Show me and I'll use it". In this preface, we present a hypothetical discussion between a consultant proposing the use of the methodology presented in this dissertation and a planner in a developing region, such as India.

Planner... How car this methodology help me?

Consultant... The proposed methodology is primarily for planners and policymakers, especially for those in developing regions. It appears to me that most planning and policymaking efforts in these regions can be related to technology transfer. Further, though it seems important that any proposed approach be suitable for a user such a yourself, most previous work on technology transfer, such as the Spencer and Woroniak study on Japan and West Germany, do not really focus on issues related to planning and policymaking. These issues, nonetheless, are of utmost concern to developing nations and are quite relevant to any approach if it is to have practical significance.

There are three aspects of these is sues that seem to be of particular concern to a user, such as yourself. These three aspects deal with:

(a) determining if the target set in the five year plans of your country are realistic, i.e. if they are realizable. 
(b) utilizing an adequate representation of technology transfer.

(c) using proper tools to analyze and understand the complex issues related to technology transfer.

Targets... First, let us deal with the issue of realistic targets. Generally, when a country such as yours undertakes a technology transfer project, it is to meet some established tragets (goals, objectives) within the country's five year plans. Often, such plans are overoptimistic and unattainable in terms of the country's capabilities. Typical of this is underestimating resource feasibility. Such an overoptimism and/or infea sibility of the established goals should be pointed out during the earliest phases of the plans. Specifically, both policy planning (i.e. setting of, and selecting from, aliernate goals) and strategic planning (i.e. selecting from the alternate ways to achieve the established goals) should utilize inputs that can convey the information concerning the realizability of the established goals and objectives. In other words, what you really need is an altering system. Such a system must be capable of drawing the attention of decisionmakers, such as yourself, to the things that can, or are likely to go wrong, i.e. identify those activities that can be 'bottlenecks'.

Previous inquiries into technology transfer do not provide you with such informational inputs. The proposed method, on the other hand, does identify the 'bottlenecks'. For instance, the method identifies explicitly the resources required for the selected technology transfer and provides information as to which resources are likely bottlenecks. 
The method also can help you as a user, in identifying the transfer channel or channels most likely to produce the desired outcomes. The consequences or the impact of technology transfer on your country's technological and nontechnological surroundings can also be assessed by the tools provided in this methodology. Thus, the outputs of this method will help you in:

(a) designing policies to overcome the anticipated, undesirable (if any) consequences of the selected transfer.

(b) proper planning and deploying of your region's resources.

(c) selecting the channel that can efficiently transfer the seiected technology, and in time to meet the set target dates. You can see that these outputs will be useful in your country's policy and strategic planning efforts.

Representation... Now, let us talk about using an adequate representation of the technology transfer process. Needless to say, it is important that the analysis utilize an adequate structure of technology transfer. What I mean is that any structure you use to represent technology transfer must contain variables relevant to the technology transfer in question and the relationship between these variables. Most previous studies have stressed economic aspects, neglecting or omitting other relevant variables such as human resources, and in particular, needed managerial skills. Yet, the very lack of the availability of such skills has often lead to operating difficulties during the maintenance phase of a technology transfer project, especially after the donor has left the 
project. The proposed methodology attempts to overcome this drawback by taking a multidisciplinary view of technology transfer. In particular, variables related to human resources are explicitly considered and their feasibility for the technology trangfer in question is examined. The results of such an examination helps you, the policymaker, to design better policies for regulation purposes.

Proper Tools... As many authors have pointed out, the issues underlying technology transfer a re complex, and tools are needed to handle the underlying complexity. In this respect, it seems quite appropriate to try and apply systems analysis, technological forecasting and policy sciences, to deal with the complexity, but maintaining simplicity of the approach. This methodology uses such tools, and incorporates a simple policy measure which is expressed as binary numbers, 0 or 1. The simpie measures and their binary specification makes the methodology especially attractive in terms of theoretical analysis and ease of computation.

Planner... Can you tell me something about the scope of the efforts requires to use your methodology?

Consultant... The scope of the efforts required to use the method in a practical situation is modest, considering the fact that a multidisciplinary structure of technology transfer is employed. The term multidisciplinary implies that the process of technology transfer is viewed not only from an economic viewpoint, which is necessary, but also by considering other relevant aspects, such as natural and human resources, 
entrepreneurial factors, and social and cultural consequences of technology (e.g. health and education). Ignoring the multidisciplinary aspects of technology transfer would lead to an inadequate representation of technology transfer and, therefore, the results derived from such a representation are of limited value. For instance, you may recall that many technology transfer projects in the public sector of many developing regions have run into acute problems precisely because the capabilities of the a vailable human and natural resources were not explicitly considered.

Furthermore, to make your task a bit easier, the user is supplied with a generalized checklist from wich he can select a list of relevant variables for the technology transfer under investigation. Having developed such a list, the user is asked to specify the interactions (relationships or dependency) between the variables. A simple, firstcut way is to specify such interactions by binary numbers. Once the interactions are specified, an operationally simple technique to synthesize the interactions is provided. Such a synthesis is then utilized to develop policy statements regarding resource feasibility, applicability of the transfer mechanisms, and the impact on the sociocultural and economic environment of your country. If you use binary specifications, you can synthesize the interactions by hand and no computer facilities are needed. This is another attractive feature of the method over other methods; for instance, if you are analyzing a technology transfer problem 
using statistical correlation techniques, your task would be quite difficult and time consuming if you do not have proper computing facilities.

Planner... How can I nisnitor the outputs of your method?

Consultant... As I mentioned arlier, the method helps you in three ways. Once you have decided to undertake the technology transfer project based on the analysis provided by this method, you can check the behaviour oi each of the variables specitied in the checklist applicable to your project during the various planned phases of the technology transfer project. By keeping watch on the indicators associated with each of the variables, you can monitor the performance not only of this method Wut also of the transfer project.

You. must keep in mind, however, that the outputs of the method can only be as good as the inputs that are provided by you, the user. Also, you must keep a close watch over the activities that were labeled 'bottlenecks' by this method.

Planner... It seems like you are professing that all aspects of technology transfer problem can be solved by your method?

Consuitant... I do not want to imply that the proposed methodology can solve al: of the problems related to technology transfer. Like most system analytic methods, such as cost/benefit analysis or contingency analysis, this method is an aid to the planners and policymakers concerned : vith technology transfer. It is not designed as a prediction tool, but ratier as a means of attaining insight for the planner. It allows 
explicit consideration of the relevant aspects of technology transfer by using judgment, and translation of mental models into mathematically logical and consistent statements.

Such a translation will permit you, the user, to construct and operate within a specified framework and force you to make the underlying assumptions explicit and, hence, the errors of analysis also clearer and explicit. Unlike some of the traditional disciplines where the truth underlying a scientific principle or the truth of empiricism can be demonstrated in laboratory models and experiments, the proposed methodology, like the methods of systems analysis, forecasting and policy sciences, can best be tested in terms of its ability to proluce information that can enable the planners and policymakers to make better decisions.

In this respect, Quade and Boucher capture the flavor of this method, when they suggest that:

"Ultimately all policies are made and all weapon systems are chosen on the basis of judgments. There is no other way and there never will be. The question is whether those judgments have to be made in the fog of inadequate and inaccurate data, unclear and undefined issues and a welter of conflicting personal opinions, or whether they can be made on the basis of adequate, reliable information, relevant experience, and cleariy drawn issues."

Planner... Umm...Well, I am not discouraged from considering more details of your method.

Consultant... O. K. Here goes... 


\section{ACKNOWLEDGEMENTS}

The author wishes to thank his dissertation supervisor, Professor Harold A. Linstone for his aid and many valuable suggestions provided in guiding the progress of this research. The author wishes to express his appreciation to Professors Lewis N. Goslin, George G. Lendaris and Roger L. Moseley for their time, interest and suggestions provided during the course of this work.

The graduate assistantship provided by the Systems Science c. program is gratefully acknowledged. 
TABLE OF CONTENTS

PAGE

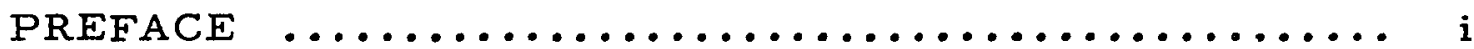

ACKNOWLEDGEMENTS $\ldots \ldots \ldots \ldots \ldots \ldots \ldots \ldots \ldots \ldots$ viii

LIST OF TABLES $\ldots \ldots \ldots \ldots \ldots \ldots \ldots \ldots \ldots \ldots \ldots \ldots \ldots \ldots \ldots \ldots \ldots \ldots$

LIST OF FIGURES $\ldots \ldots \ldots \ldots \ldots \ldots \ldots \ldots \ldots \ldots \ldots \ldots \ldots \ldots \ldots \ldots \ldots$

CHAPTER

I INTRODUCTION $\ldots \ldots \ldots \ldots \ldots \ldots \ldots \ldots \ldots \ldots$

1.1 The Importance of $\mathrm{TT} \ldots \ldots \ldots \ldots \ldots \ldots . \ldots 3$

1.2 TT Problem $\ldots \ldots \ldots \ldots \ldots \ldots \ldots \ldots \ldots$

1.3 Generating Questions $\ldots \ldots \ldots \ldots \ldots \ldots \ldots 11$

1.4 Relationship of TT Problern to Planning and Policymaking .................. 12

II A REVIEW OF PREVIOUS ST UDIES ON

TECHNOLOGY TRANSFER ............. 16

2.1 Descriptive Approaches ..............17

2.2 Analytical and Empirical Approaches ......20

2.3 Network Approach ...................30

III THE PROPOSED INQUIRING SYSTEM:

INTEGRATIVE, UNIFIED APPROACH TO

TECHNOLOGY TRANSFER ............... 35 
3.1 Objectives for Developing the Methodology ................... 36

3.2 Requirements to Realize the Objectives... . 30

3.3 Assumptions ..................... 41

3.4 The Proposed Framework of the IS for TT.. 42

3.5 A General, Basic Model of the IS for T T... 44

3.6 Development of the Hierarchical Structure . 46

3.7 Level 1: Morphological Tree ........... 49

3.8 Level 2: Interaction .............. 55

3.9 Level 3: Design................... 76

3.10 Performance Measures for TT......... 78

IV APPLICATION OF THE PROPOSED IS:

ZANSFER OF COMPUTER TECHNOLOGY

TO INDLA $\ldots \ldots \ldots \ldots \ldots \ldots \ldots \ldots \ldots . \ldots . \ldots . \ldots . \ldots . \ldots$

4.1 Selection of Technology for Transfer, Donor and Recipient Countries......... 95

4.2 Application of the Methodology.......... 96

4.3 Analysis and Cornments on the Synthesized Matrices of Round 1 .............. 140

4.4 Development of Levels 1 and $2 \ldots \ldots \ldots \ldots . .141$

4.5 Comments on the Synthesized Matrices (Round 2) ...................... 147

4.6 Performance Measures for CT Transfer to India ........................ 151

4.7 Level 3 Requirements .............. 170 
4.8 Implications for Planning and Policy-

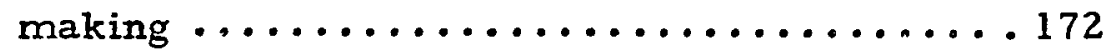

V. CONCLUSIONS AND EXTENSIONS ........... 189

5.1 Conclusions $\ldots \ldots \ldots \ldots \ldots \ldots \ldots \ldots$

5.2 Extensions .......................... 199

REFERENCES ........................... 200

APPENDIX A $\ldots \ldots \ldots \ldots \ldots \ldots \ldots \ldots \ldots \ldots \ldots \ldots \ldots \ldots \ldots \ldots . \ldots \ldots$

APPENDIX B ............................. 210 
Interaction Matrices: Interpretation and

Specification ......................6 67

II Justification for Selecting Variables in

Level 1, Round 1.................... 103

III Explanation of Entries in the Interaction

Matrices (Round 1) .................... 124

IV Revised Interactions for Round $2 \ldots \ldots \ldots \ldots \ldots \ldots$

$\mathrm{V}$ Specifications for the Measures: a-Availability;

b-Productivity; c-Cost .................. 152

VI Policy Statements for TT Feasibility .......... 155

VII Specification of Applicability Measures ........ 161

VIII Policy Statements for Applicability of Transfer Mechanisms: Software ................ 164

IX Policy Statements for Applicability of Transfer Mechanisms: Hardware and Peripherals ....... 165

X Comparison of the Proposed Methodology with Other Approaches .................... 192 


\section{LIST OF FIGURES}

FIGURE
Some Aspects of Technology Transfer .........10

Planning: The Ideal Setting ...............14

Some Descriptive Models of TT............ 19

Technology Gap of the "Disembodied" Type ...... 24

Technology Transfer Model: Network Type ..... 31

Technclogy Transfer: Relational Concepts ...... 38

Basic Framework for Developing the IS for TT...44

The Proposed Hierarchical Structure of TT ......48

Simplified, Overall Flowtraph of the Proposed

Method ......................... 50

Level l: A Generalized Morphological Tree for $\mathrm{T} T$

Flowgraph: Level $1 \ldots \ldots \ldots \ldots \ldots \ldots \ldots$

Possible Interactions Between Technology, Resources, Mechanisms and Consequences ...... 57

The Proposed Structure of $\mathrm{TT} \ldots \ldots \ldots \ldots \ldots 6$ Level 3: Design Process for Technology Transfer 77 Possible Policies for TT Feasibility Assessment... 81 Plan for Demonstrating Application of the Proposed Methodology .................. 94 
Morphological Tree for CT, Round 1.........101

Number of Computers Installed .............. 114

The World Computer Industry in 1966 and 1969... 115

GNP and Number of Computers ........... 118

Development of Computer Usage: France vs

U.S.A. ........................... 120

Socio-Economic Status of Nations ............ 122

$\left(T_{x R}\right)_{f}$ Matrix, Round 1 ............... 137

$(\mathrm{TxM})_{\mathrm{f}}$ Matrix, Round $1 \ldots \ldots \ldots \ldots \ldots \ldots \ldots \ldots \ldots$

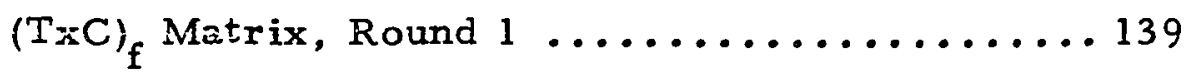

Revised Morphological Tree, Round 2 ....... 142 


\section{CHAPTER I}

\section{INTRODUCTION}

The concept of technology transfer has been around for quite some time; yet, the meaning of the phrase depends on the audience considering it and the point in time. For the purposes of this dissertation,

technology transfer ${ }^{1}$ will denote the purposive movement of established technology of technical innovation from place to place, company to company, use to use. Technology, as understood here, involves not only hardware (machines, tools) but also sofeware (PERT, Algorithms). Several definitions of technology and TT are given in Appendix A. The inquiry into $\mathrm{T} T$ is carried out here in a systematic manner as outlined by the following steps:

1. The importance of $T T$ is explained, followed by an examination of the dimensions of the TT problem.

2. The previous studies on TT are reviewed to determine the nature, scope and limitations of the previous inquiries.

1. Technology Transfer will be abbreviated TT in the rest of this discussion. 
3. Based on the above two steps, a new framework for understanding and analyzing TT is suggested and discussed.

4. The suggested methodology is applied to study the transfer of computer technology to India, and results of the application are examined in terms of policy and strategic planning, system regulation and improvement.

Before discussing the importance of $\mathrm{TT}$, three terms frequently used in this dissertation will be explained. These are:

1. Inquiring System.

2. Vertical and Horizontal Transfer.

3. Transfer Mechanisms.

Inquiring System: This term is used here in the ense discussed by Churchman [21,P.8] : "inquiry is an activit́y which produces knowledge". Five specific types of inquiries are discussed by Churchman: Leibnizian, Lockean, Kantian, Hegelian and Singerian. A detailed and inspiring discussion of these inquiring systems can be found in Churchman [21], and a shorter version in reference 64. For our purposes, the term inquiring system will signify the "creation of an ability of the human being to solve his problems" in a changing environment. In this sense, an inquiring system can be called a model, framework, methodology, or an approach. These terms will be used interchangeably to represent the concept of inquiring system.

Vertical and Horizontal Transfer: Brooks [17] has suggested two dimensions of TT: Vertical and horizontal. Vertical transfer involves 
the application of a general principle to produce a new product, device or process within a given scientific or technological discipline and generally within an organizational entity. Jantsch $[43, \mathrm{p} .24]$ mentions eight levels of vertical TT. The horizontal transfer can occur at each vertical level. In horizontal TT, one technology is adapted to a different area of application, generally across institutional lines. Since both vertical and horizontal TT are time dependent, a third dimension of time can be added to the two -- horizontal and vertical -- dimensions, yielding a three dimensional TT space.

Transfer Mechanisms: There are a number of mechanisms to transfer technology. It is fruitful to visualize a transfer mechanism as an information channel which connects a sender (donor organization) to a receiver (recipient organization). The channel for transfer can be: government, business, person or a journal. It can be visualized intuitively that the characteristics of a specific TT are affected by the transfer mechanisms utilized for that $T$ T.

\subsection{The Importance of TT}

It seems worthwhile to discuss why $\mathrm{TT}$ is an important topic for serious study. One way to illustrate the importance of $\mathrm{TT}$ is to consider the notion of "technology gap". Denison [25] has shown that a technology gap does exist among nations. The Atlantic Institute has performed a study identifying and determining the causes for a technological gap between the U.S. and Europe [94]. A purposive TT can help reduce the 
technological gap by providing strategies to overcome deficiencies of the prevailing technologies.

In recent years, interest in TT has been accelerated as a result of developing countries' need to employ technology to produce structural change. Also, the developed countries seem anxious to diversify their capabilities through mechanisms such as multinational corporations. National or regional development means growth plus structural change rather than growth alone. Many writers have pointed out the importance of technology to produce structural change. For instance, Schon notes $[82, \mathrm{p} . \mathrm{xv}]$ :

\footnotetext{
"on still another level it is part of the new conventional rotion that technological innovation is essentia? to economic growth and that economic growth -- as experienced by rate of increase in Gross National Product -- is a sign of national economic strength and vitality".
}

The UNESCO conference on the application of Science and Technology to the development of Asia recommended that $\mathrm{TT}$ and information centers be set up urgently in order to provide assistance to enterprises identifying their technological needs, ascertaining the a vailability of necessary technology abroad and negotiations of the agreements [96,p.216].

Given that a technology exists and there is a desire to employ it, two choices are available to a company, a region or a nation: (a) to develop the technology from the lowest vertical TT level(s), or (b) to borrow the existing technology from a donor company, region or nation (horizontal transfer). Many nations have found it profitable to borrow technology because of a combination of factors such as production, 
resources and finances, to name a few. For instance, Japan has actively and consciously borrowed technology. The importance of TT in this respect seems evident.

There is yet another consideration for emphasizing the importance of TT. It is possible that technology may not always generate proper structural change. Technology is a source of uncertainty [95,p.1]. The interaction of society and technology is understood poorly. Mesthene notes $[61, \mathrm{p}$. viii]:

"The strains that technology places on our values and beliefs finally are reflected in economic, political and ideological conflict. That is, they raise questions about proper goals of society and about proper ways of pursuing those goals".

Bauer [13] considers second-order consequenc ss of technology and $\mathrm{T} T$. Ozbekhan states $[69$, p.61]:

"Yet another characteristic of technology is that although its costs are systemwide, the gains never seem to add up to system wide improvement or betterment".

Such concerns have led to the establishment of groups to assess the impact of technology. The utilization of TT in reducing technological gap and producing structural change should result in system improvement. A study on $\mathrm{TT}$ can enlighten those facets of particular relevance to system improvement.

Finally, it is important to investigate the role of TT in policyrnaking and planning activities of developing regions. Since policymaking and planning in these regions is heavily dependent on $T T$, it seems essential to consider TT as the most vital part of the planning and policymaking 
efforts. The methodological questions -- "why, what, who, how and when to transfer" -- form the heart of planning and policymaking efforts in these regions. For instance, the five-year plan of India deals, to a large extent, with the questions related essentially to TT [18,pp.305-315].

\subsection{TT Problem}

Inspite of the importance of $T T$, the literature on the subject indicates a lack of agreement concerning the major components of the TT problem. The lack of such a consensus can best be illustrated by providing a brief review of the attempts to decompose the problem. Gruber and Marquis $[35, \mathrm{p} .4]$ identify three problem areas of concern: (a) innovation, i.e., the utilization of technology, (b) the process of development of technology, and (c) government influences on the diffusion of technology.

Bar-Zakay [12] summarizes the overall TT problem, by extending Dror's [28] statement on problems related to public policymaking, as follows :

"Scientific and technological information relevant to developing countries is becoming more and more a vailable. How can this information be introduced into the technology transfer process so as to increase the correlation between knowledge and power?"

Bar-Zakay then decomposes the overall TT problem into the following subproblems:

(a) Problem related to inter-context information. This subproblem refers to the limitation on scoiety's use of information; the 
source of limitation lies in society's inability to put to use the information originating in one context into a new context to have a new meaning.

(b) Resources problem which refers to society's capacity to produce information quite different from its capacity to digest informa tion. It has been observed that the developing countries have not benefitted from the vast amount of information generated at high cost by the developed countries.

(c) Awareness problem refer s to making educated decisions concerning choices available. A good TT should recommend or implement an educated decision. The TT must be planned or organized, i.e., triggered by decision rather than action.

It is important to note that a synthesis of the above subproblems should result in a "total greater than the sum of its parts".

Svenilson [87,p.176] suggests three broad elements of the TT problem:

(a) Social systems and human attitudes.

(b) Knowledge and human skills.

(c) The physical elements in which modern technology is

embedded.

Although such a breakdown is too general to be useful, it can be utilized to represent three separate dimensions of TT.

One can observe from the above discussion that a suitable decomposition of the TT problem does not exist. A useful way to decompose 
the problem is to consider five essential aspects of any $\mathrm{T} T$ problem. These five àspects are:

(a) Structure: A structure represents the elements (variables) of the process together with their relationships. Specification of the structure of a complex system is usually based on an understanding of the underlying phenomena and justifiable assumptions regarding the nature of process variables and their relationships. Forrester's work $[29,30,31]$ points out quite well the usefulness and the importance of developing the structure of complex systems.

(b) Time factor: The time to transfer technology is an important consideration for any $T \mathrm{~T}$ problem. In fact, "time to transfer" can be specified as a performance measure for assessing the worthwhileness of TT. Svenilson $[87, p, 176]$ expresses the importance of the time factor as:

\footnotetext{
"In any case, the problem of transfer of technology is one of speed. The main question is: Can the transfer of modern technology occur within a few decades instead of a long-run secular process? ... But the emphasis should be on the time element involved. Only in this way will our research correspond to operational needs".
}

The seriousness of the time element varies with the specific TT being investigated. For instance, India cannot afford the luxury of a long time to transfer technologies such as food production and processing because of the rapidly changing burden of domestic population.

(c) Initial and Terminal Conditions: It also seems important to consider the nature of the two boundary values for any $\mathrm{T} T$ problem -- 
initial and terminal. The selection of initial and terminal conditions can affect the performance of T'T process.

(d) Impact or Consequences: The impact of the transferred technology on the recipient's technological and nontechnological environment should preferably be considered as part of the overall TT problem. This aspect of TT is currently known as technology assessment.

(e) Technological Forecasting: The problem of TT is linked closely with technological forecasting. For instance, technological forecasting is related to and needed for all four aspects of the TT problem mentioned above. Both exploratory and normative forecasts are necessary. However, it appears that the methodology for forecasting horizontal TT is not fully developed yet [43,pp.109-132]. The problems related to technological forecasting thus become the subproblems of TT. The relationship of $\mathrm{TT}$ and technological forecasting is discussed extensively in references 43 and 44.

The above aspects, to some extent, can be illustrated as shown in Figure 1. In this Figure, a TT (horizontal or vertical) begins at time $t_{1}$ and ends at time $t_{2}$. The time interval $\left(t_{2}-t_{1}\right)$ is the time required to transfer the selected technology. The trajectory from $t_{1}$ to $t_{2}$ shows how the TT process can progress horizontally, and/or through vertical levels. The shape of the trajectory depends on the conditions prevailing at times $t_{1}$ ard $t_{2}$, and available technology forecasts for the time period $\left(t_{2}-t_{1}\right)$. The dashed trajectory is drawn to indicate that alternate ways to reach the ands exist. 


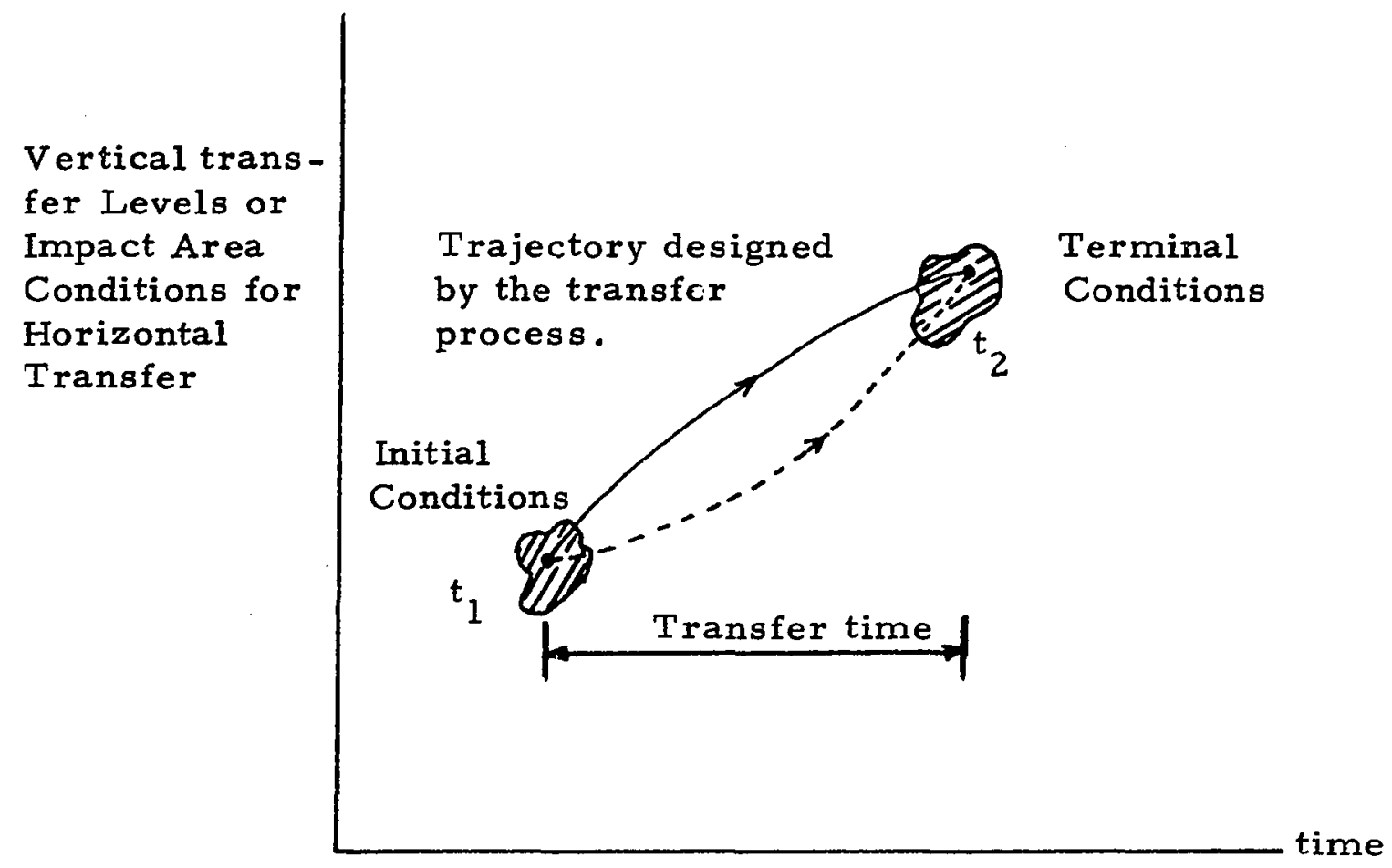

Figure 1. Some aspects of the technology transfer problem 


\subsection{Generating Questions for the TT Problem}

It seems that the nature and the complexity of $\mathrm{T} T$ problem can best be explained in terms of generating questions. The term "generating questions" is due to Ashby [8]. Quade and Boucher [p.212] also indicate the importance of generating questions in systems analysis. Generating questions e erve two purposes: (a) they specify the nature of answers required from a model or investigation, and (b) they help identify variables relevant to the problem structure.

For the TT problem, a list of generating questions can be:

1. What are some of the characteristics of transfer of technology?

2. Who undertakes $\mathrm{TT}$ ? Under what circumstances?

3. What transfer mechanisms or channels have been employed? With what success? HCW can sne assess the applicability of a particular mechanism?

4. What is the most effective TT? How can one nueagure the success of a transfer?

5. What policies can the recipient and the donor organizations adopt to promote more efficient TT?

6. How can one ensure that $\mathrm{TT}$ is feasible?

7. What is the structure of the TT process?

8. What are the management and human factors related to TT?

9. How can one assess the impact or determine the conse- 
10. Are the existing institutions in the developing countries performing their functions so as to promote and facilitate TT?

11. What is known about the incentives and barriers to TT?

The above list can possibly be extended. However, it contains the core questions needing answers. Although it would be imfossible to answer all of the above questions in a single study, we intend to consider those questions that seem especially important; these are related to the structure, feasibility of $\mathrm{TT}$, applicability of transfer mechanisms, assessing the consequences of the transferred technology and means to generate effectiveness scales.

1.4 Relationship of TT Problem to Planning and Policymaking

As the title of this dissertation suggests, one major concern here is the role T T plays in planning and policymaking. Hence, planners and policymakers concerned with $\mathrm{TT}$ are assumed to be the users or clients of the methodology described here. There are some important aspects of TT problem related to these users.

The first important aspect is that the methodology must be operational and communicable to the users. Often, these clients, especially in a developing country, do not have a serious theoretical background in systems sciences. Thus, the communication process is important. Also, the simplicity of the approach to TT is essential.

Secondly, the methodology must be useful as a decisionmaking aid. If a TT does not provide system improvement, its usefulness is small 
indeed. Policy implies system regulation; regulation in turn requires that purposive decisionmaking exists. Therefore, in designing the approach to $\mathrm{TT}$, the decisionmaking needs of the clients must be kept in rnind.

Thirdly, it has been suggested by Michael [62] that most planning efforts are resisted. Thus, the TT problem must consider this important consideration of resistance to change. Several suggestions have been made to counter the resistance to change (e.g., creating minicrisis); these suggestions, however, do not provide hard prescriptions. The best one can do here, at the present time, is anticipate the consequences of TT so thai: policies can be designed to reduce the resistance.

Firally, the phenomenon of discounting the future is present. This is more so in developing regions where long-range goals are discounted heavily in favor of the short range ones. Linstone [55] discusses this problem by considering the effects of discounting on the Forrester Meadows World Dynamics model. One useful way to counter the discounting phenomenon, as far as $\mathrm{TT}$ is concerned, is to employ a proper mix of short-range and long-range goals. The process of generating such a mix of short-range and long-range gcals would force the planner to consider explicitly the discounting phenomenon.

Although we do not propose to solve all of the above problems here, it seems useful to keep these problem aspects in mind for developing a possible inquiring system for $T T$. In paricular, let us use the ideal framework shown in Figure 2 [54] as a background for considering 


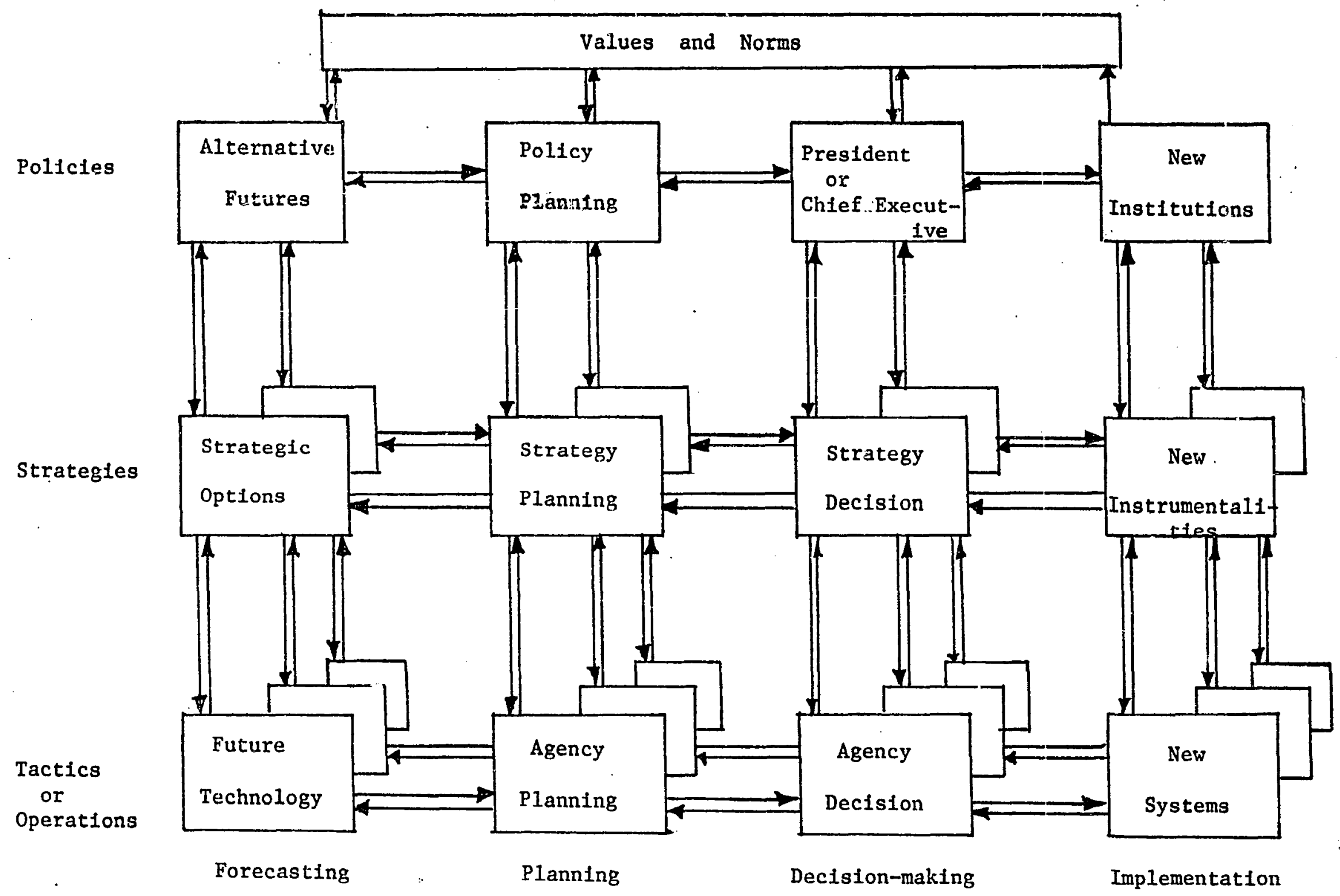

Note: Not A1I Arrows Shown

Figure 2. Planning: the ideal setting 
the TT problems related to planning and policymaking . Figure 2 considers the relationships of the three levels of planning-policy, strategic and operational - with each other, as driven by norms, which in turn, are derived from values. The relationships of the processes of forecasting, planning, decisionmaking and implementation to the three levels are also shown in Figure 2. The three levels are not connected via 'one-way' paths: rather they exchange information through various feedback loops. These three aspects of planning, the dominance of norms and values in planning and policymaking and the feeding back of information provide useful basis for the development of the profosed methodology. 
CHAPTER II

\title{
A REVIEW OF PREVIOUS STUDIES ON TECHNOLOGY TRANSF ER
}

\begin{abstract}
A review of previous studies on $\mathrm{TT}$ (Technology Transfer) seems essential before outlining a new and a different approach to the problem. Such a review serves two purposes: (a) it shows the contribution and limitations of previous approaches, and (b) it provides some basis for the approach proposed in this study. Thus, the subsequent discussion provides a link between the previous discussion and the proposed approach to $\mathrm{T} T$.
\end{abstract}

A glance through the reference section of this thesis indicates the extent of work done on technology transfer and related problems. In terms of the concern of this study, the previous approaches can be conveniently grouped into three broad categories:
(a) descriptive approaches.
(b) analytical and empirical approaches.
(c) network approach.

These approaches will now be discussed. 


\subsection{Descriptive Approaches}

Most of the previous work on technology transfer falls under this classification. Descriptive approaches discuss rather broadly the important aspects of technology transfer. Brooks [17] suggested the notions of vertical and horizontal technology transfers as mentioned in Chapter I. Spencer, who has written extensively on the subject of technology transfer, provides a descriptive framework for studying technology transfer mechanisms and problems as applied to developing countries and suggests establishing a universal body to organize and facilitate TT to developing countries [89]. References 87 and 35, both the results of conferences on $\mathrm{TT}$, contain several papers which discuss economic, information, human factors and entrepreneurial aspects of technology transfer. Reference 87 , heavily oriented towards economic aspects, does not give concrete conclusions regarding technology transfer. Notable in this reference is the emphasis on the discussion of Schumpeter's theory of economic growth suggesting a need to treat TT process in terms of stagewise growth patterns. However, the view of the conferees was that Schumpeterian theory is not very applicable to the study of TT. Reference 35, which is more interdisciplinary in nature and contents, also fails to provide a prescriptive or unified approach to TT.

Notable i1: reference 35 is a paper by McClelland discussing the role of achievement orientation in TT. McClelland refers to "need for achievement" by the term "n-Ach" and points out that this factor is 
more common among innovator entrepreneurs in business world than professionals. Such observations lead to speculation that technologies developed by government and university laboratories may not lead to innovation.

The participants of the MIT conference on technology transfer suggested three descriptive models for viewing TT [35, pp.4-8]. These are shown in Figures $3(\mathrm{a})$, (b), and (c). Model I examines the relationships among the major channels of activity and provides a topological relationship between science, technology and the ultimate uses of science and technology. The model shows various communication paths and indicates the possible sources of idea generation. Model II examines the sequence of events within the organization structure of a given potential recipient or user of existing technology. Model II has two links, with the outside world, one at the point of entry (i.e. the box marked 'Idea') and one at the point of marketing the inventive effort (i,e. diffusion). Model III does not have a direction to it. The independent variables can often be related to one another and a given problem solved simultaneously, rather than sequentially. Model III often gives insight into Model II.

The above three models were proposed on empirical, intuitive feelings of the conferees. Hence, it is difficult to label them as a specific inquiring system. However, the spirit of such inquiries is Lockean in nature, striving for Leibnizian description.

The most noticeable drawback of descriptive approaches to TT is that they provide verbal understanding rather than guidance to decisionmaking and action. Furthermore, they fail to provide a unified view 


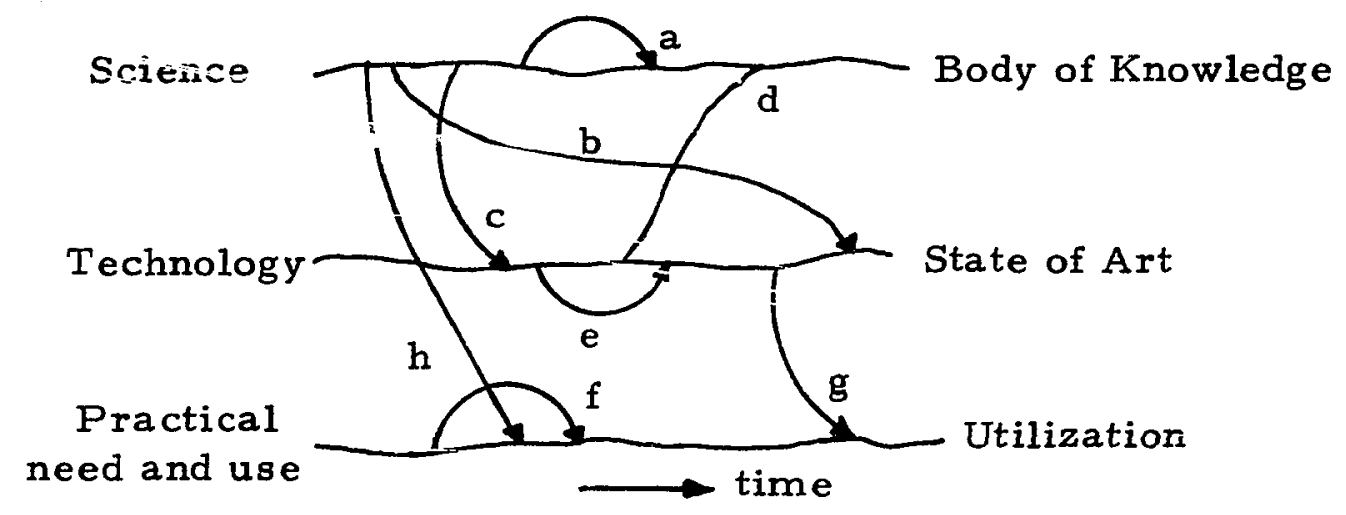

Figure 3(a). Model I: Science, Technology and Utilization of Their Products Showing Communication Paths Among the Three Streams

Path (a) Science to Science (Slow)

(b) Science to Technology (Slow)

(c) Science to Technology (Fast Gap Filling)

(d) Technology to Science (e.g. Instruments)

(e) Technology to Technology

(f) Use to Use

(g) Technology to Use

(h) Science to Use

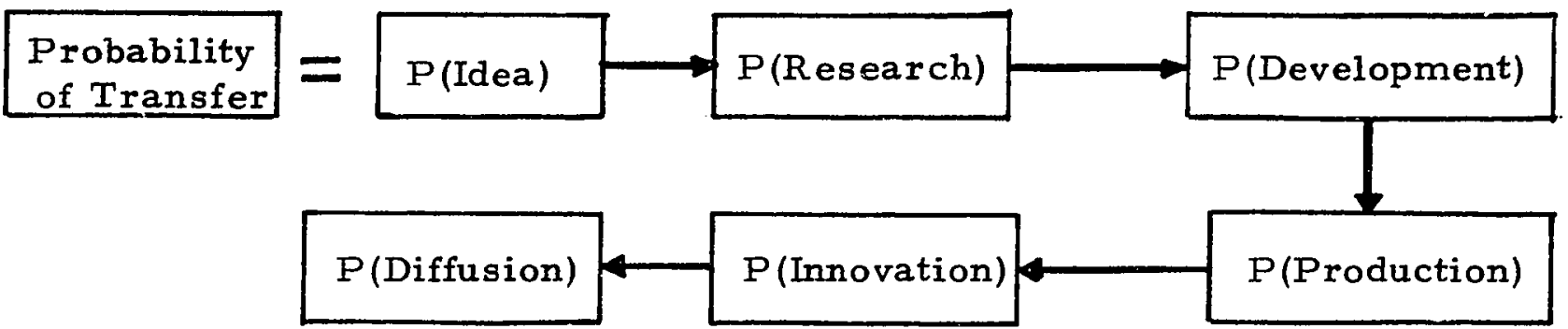

Figure 3(b). Model II

Magnitude of $=f$ (Source, Nature of Item to be transferred, Structure Transfer of channels for transfer, Potential Recipients (customers) of item to be transferred)

Figure 3(c). Model III 
of TT. It was mentioned in Section 1.4 that the total problem of TT must consider decisionmaking and implementation aspects which in turn demand prescriptive remedies. The major contribution of the descriptive approaches has been in putting $\mathrm{T} T$ in perspective and in increasing the understanding of TT problems.

\subsection{Analytical and Empixical Approaches}

\subsubsection{Analytical Approaches}

Three analytical approaches seem sign:- for the present discussion:

(a) models of technological progress in terms of industrial productivity, profitability and patents derived by Minasian, Schmookler and Mansfieìd.

(b) a model developed by Kmenta utilizing the notion of tech nology gap.

(c) a model developed by Spencer and Woroniak involving the concept of TT function.

The empirical approaches are Lockean in nature while the analytical ones are attempts at Leibnizian inquiries. 


\subsection{1.(a) Models of Technological Progress}

Schmookler [81] has carried out extensive research on patent statistics. Schmookler utilizes regression models to correlate patent activities with some variables that can be considered as inputs to inventive activities. Schmookler's model then describes gross technological progress in terms of these variables. Schmookler also attempts to correlate economic activity with technology. Two major conclusions resulting from Schmookler's work are: (a) technological progress is driven by economic progress rather than vice versa, and (b) the percentage increase in economic investment is related to the percentage increase in patents and that value added in an industry correlates well with patents. These conclusions indicate that patents can be taken as a proxy for investment. Thus, it has some application to TT problem.

Major shortcomings of Schmookler's model are: (a) the regression equations cannot be applied to all types of industries, and (b) patents may not be a good index. It has been determined that presently patents no longer provide a good mechanism for TT (as was the case in past) because companirs fear that the patents publicize the invention to the detriment of their interests. Brooks [17] indicates that patents are declining in importance as means of $\mathrm{TT}$ and are not a significant basis of new enterprises or sophisticated technology. Also, the data indicate that the number of patents are declining relative to $R \& D$ expenditures. The major contribution of Schmookler's work appears to be in technological forecasting activities related to $\mathrm{TT}$ and in relating measures of investment with technological growth in economy. 
Minasian [63] developed his model to test the hypothesis that $R \& D$ expenditures are related to growth in productivity. Thus, an increase in $R \& D$ expenditure would imply a growth in productivity. Minasian's analysis using extensive industrial data supports his hypothesis. However, his sample of industries was not taken at random, rather it was selective. Thus, his results may have a limited significance. Again, Minasian's results have limited usefuiness in $\mathrm{TT}$, since they indicate factors responsible for growth in productivity.

Mansfield [57] has developed a model which is similar to Minasian's except that profitability is related to $R \& D$ expenditures. Both productivity and profitability can be taken as measures of technological growth and hence, are useful in a study of TT.

These studies represent significant analytical efforts in attempting to structure the problem and identifying the factors, such as patents, productivity, profitability and $R \& D$ expenditures, responsible for technological growth. In a way, the results of these models support the findings of the project Hindsight [4l] that mission oriented research is more worthwhile and profitable. The major limitations of these models are: limited applicability to TT, and methodological difficulties of using them. Also, the results should be interpreted cautiously. However, these models are of some use in $\mathrm{TT}$, since one problem faced by developing countries is to determine whether to buy $R \& D$ services as part of $T T$. 
2.2.1. (b) Model based on the Notion of Technology Gap

Kmenta [47] defines technology gap as "disembodied" or "embodied" type. If the same quantities of inputs produce a higher level of outputs in a country A than a country $B$, the technology gap is of "disembodied" type. If a country $A$ is superior technologically to a country B as a result of possessing superior inputs, the gap is "embodied" type. The technology gap of "disembodied" type can be represented as shown in Figure 4, assuming two inputs -- labor and capital, and a homogeneous production function. Lb/Y represents labor per unit output while $\mathrm{K} / \mathrm{Y}$ represents capital per unit output. If the labor is relatively expensive in the country A (e.g., U.S.) and relatively cheap in the country B (e.g., India), one would expect the actual combinations of labor and capital to look something like $A *$ and $B *$. The extent of the "disembodied" technology gap can be measured by the distance between the two curves at a particular labor/capital combination, i.e., along a particular ray from the origin.

Using the notion of technological gap, Kmenta proposes a simple lag model of the form:

$$
B(t)=A(t-L)
$$

where $A(t), B(t)$ refer to the level of technology in countries $A_{4}$ and $B$ at time t. L is the number of time periods by which $B$ lags $A$ at time $t$. L can be expressed in terms of institutional variables related to patents, international movements of inputs, external military presence, etc. The 


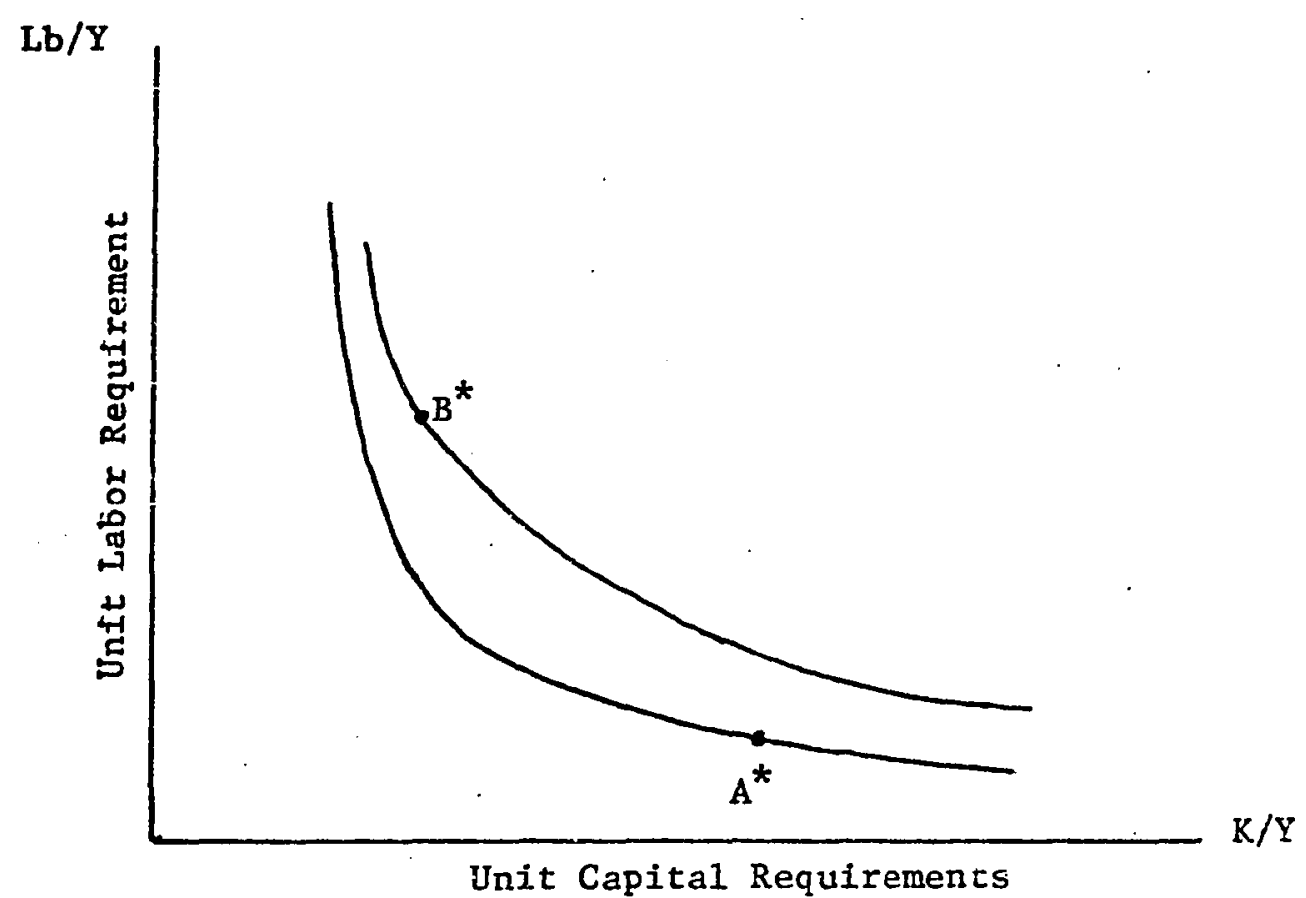

Figure 4. Technology gap of the "Disembodied" type 
traditional view that technology grows with time implies the possibility that country B could catch up with country A. For instance, let

$$
A(t)=A_{0} x^{t}
$$

Then:

$$
\frac{A(t)-B(t)}{B(t)}=x^{L}-1
$$

From (2.3), it follows that country B can catch up with country A only if:

(a) $L$ is decreased, i.e., the rate of technology growth in country A is slowed down, or (b) $x$ is reduced.

Kmenta also considers technology growth with a limit by modifying equation 2.2. In this case, it is found that country B can catch up with country A regardless of the lag. Kmenta also considers adaptive production functions and learning curves to represent the two countries' inputoutput behavior over time.

Kmenta's models can be useful to identify the factors which can facilitate TT and reduce the gap. However, as Mansfield points out in his comments on Kmenta's paper: "these models deal only with minor part of the problem of explaining the size and behavior of technology gap, since they investigate the effects of the speed of adjustments of the gap, taking the speed as given". The factors influencing the size and the behavior of the gap are not considered. Human and social factors are also excluded. Nonetheless, Kmenta's work can be useful in studying the rate of international diffusion of technology. These models have not been applied to any 'real world' problems. 


\section{2 .1 (c) TT Functions}

Spencer and Woroniak $[88,90]$ have developed what they term technology transfer functions. They have determined the nature of these functions for two cases, Japan and West Germany. The TT function is made up of two parts: (a) impact function, and (b) absorption function. The term "impact" here is taken in a narrow sense, implying impact on export and domestic sales. The term "absorption" indicates the total annual royalty remittances to foreign firms for borrowing technology. Spencer [88] develops the following equations for Japan:

$$
\begin{aligned}
& E F_{t e}=a+b_{1} E W+b_{2} \frac{P_{w}}{P_{j}}+b_{3} T C R+b_{4} T_{r m}+b_{5} M T T_{m c h-1}+b_{6} R D+b_{7} A \\
& S D_{t e}=a+b_{1} N Y+b_{2} C C+b_{3} M c+b_{4} M T_{r m}+b_{5} M T T_{m c h-1}+b_{6} R D+b_{7} T C R+b \frac{I}{8 I} \\
& T C R=a+b_{1} N Y+b_{2} F X+b_{3} M P+b_{4} D e+b_{5} R E+b_{6} P a+b_{7} R D-1+b_{8} A
\end{aligned}
$$

where

$$
\begin{array}{ll}
\mathrm{EF}_{\mathrm{te}} & =\text { Japanese exports related to new technology; } \\
\mathrm{SD}_{\mathrm{te}} & =\text { Domestic sales related to new technology; } \\
\mathrm{TCR} & =\begin{array}{l}
\text { Total annual royalty remittances to foreign firms for borrowing } \\
\text { technology; }
\end{array} \\
\mathrm{EW} & =\text { World exports; } \\
\mathrm{P}_{\mathrm{w}} & =\text { World manufacturing export price index; } \\
\mathrm{P}_{\mathrm{j}} & =\text { Japanese manufacturing export price index; } \\
\mathrm{MT}_{\mathrm{rm}} & =\text { Imports of raw material related to new technology; } \\
\mathrm{MT} & \text { Imph-1 }
\end{array}
$$




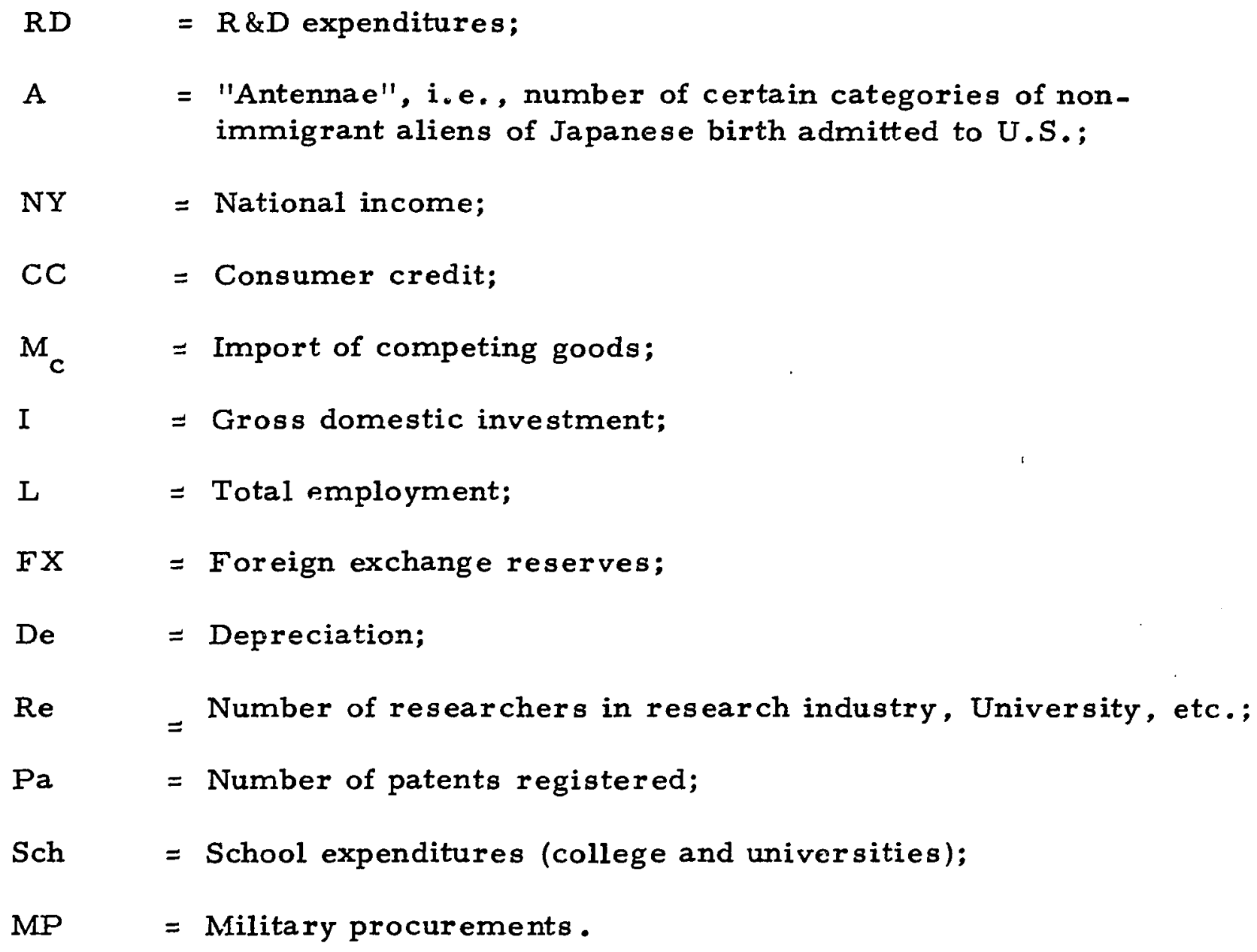

The equations (2.5) and (2.6) represent impact function. Using available data and employing regression techniques, Spencer determines the foll owing relationships as significant:

$$
\begin{aligned}
& E F_{\text {te }}=f\left(E W, M T_{\text {mch }-1}, R D\right) \\
& S D_{t e}=f\left(I / L, M T_{r m}, R D\right) \\
& T C R=f\left(N Y, F X, D e, R e, P a, A, S c h, R D_{-1}\right)
\end{aligned}
$$

In (2.9) TCR has inverse relationship with $\mathrm{FX}, \mathrm{RD}, 1$, A and Sch.

Based on such analysis, Spencer and Woroniak suggest that the variables affecting $\mathrm{TT}$ can be grouped into four categories as those representing: 
(a) technological and educational base.

(b) society's planned and predetermined efforts.

(c) coordinated policies of the government.

(d) antennae -- i.e., exposure to new, foreign technology.

Some of the critism of other approaches applies to this model also, i.e., a lack of explicit treatment of social and human factors. Also, regression equations do not yield cause/effect information, since the presence of strong correlation between two variables does not convey information concerning the independency of the two variables. There are also the methodological difficulties in using these models, especially by planners and policymakers. For instance the study concludes that TCR is not a good proxy for $\mathrm{TT}$, yet the conclusions are based on the behavior of TCR.

On the positive side, the model does identify some of the significant economic variables useful in studying any $\mathrm{TT}$. Of course, the significance of these variables will vary from one country to another.

\subsubsection{Empirical Approaches}

There are four approaches that must be mentioned here. Doctors has performed two studies $[26,27]$ involving the role of federal agencies in TT. Roberts [75] has performed a study on entrepreneur and technology, using 200 companies on Route 128 (near Boston, Mass.) founded by the ex-employees of MIT. Allen [4] has performed a study on information channels utilized by the personnel related to $\mathrm{TT}$. 
Doctors' study on the role of federal agencies in $\mathrm{TT}$ [26] considers federal government's programs initiated to facilitate TT. Doctors draws several conclusions and makes recommendations regarding these programs. He finds: (a) the program is far too fragmented, (b) existence of significant political pressures, (c) major emphasis on information dissemination, (e) failure of patent and license policy, and (f) lack of successful mechanisms for horizontal TT. His recommendations include revising the framework within which such programs ope rate. In another study [27], Doctors finds that NASA efforts to transfer technology has not yielded any appreciable results. He concludes that the present NASA program has not generated transfer equal to its costs, either in terms of specific items or in the more general area. Nor has the program done what it might to use even its negative results to increase our understanding of the horizontal technology transfer $[27, \mathrm{p} .10]$.

In his study on entrepreneurships, Roberts [75] found that "differ ences between mere technical invention and successful innovation is largely attributable to the personal role of the entrepreneur". In both large corporations and new firms, Roberts found the entrepreneur to be a man of youth and advanced technology based education aisd canis from an environment in which the exploitation of technical ideas is being pursued. Large corporations limit the abilities of young entrepreneures and consequently new forms of organizational incentives and philosophies are needed to stimulate the would-be entrepreneur. 
Allen [4] attempts to identify the information sources that $R \& D$ engineers use and the impact of such information on the quality of research being performed. Such a knowledge would help predict the effects of potential changes in the information services used by scientists and engineers. Allen studied six channels of transferring technical information. Data were gathered through available records and extensive interviews with engineers. Allen found serious misalignment between the quality of ideas gathered through the channels and frequency with which the channels are used by engineers. He also found that literature is not generally used, that better performers rely more on the information generated within the laboratory and mismatch in coding schemes causes ineffective communication.

The empirical approaches, although not applicable to all sectors of $\mathrm{TT}$, add to the understanding of the process of $\mathrm{TT}$. Most of the results are derived for selected segments of technology and thus have a limited application in deriving a framework for analyzing TT problems.

\subsection{Network Approach}

The only network model suggested for technology transfer is due to Bar-Zakay [11]. His model is shown in Figure 5. The model is based on the notion behind PERT (Program Evaluation and Review Technique) that no activities can start before all activities and inputs leading into it have been completed. The attempt to contain all important facets of TT in a single model could classify this approach as Leibnizian. The important features of this model are: 


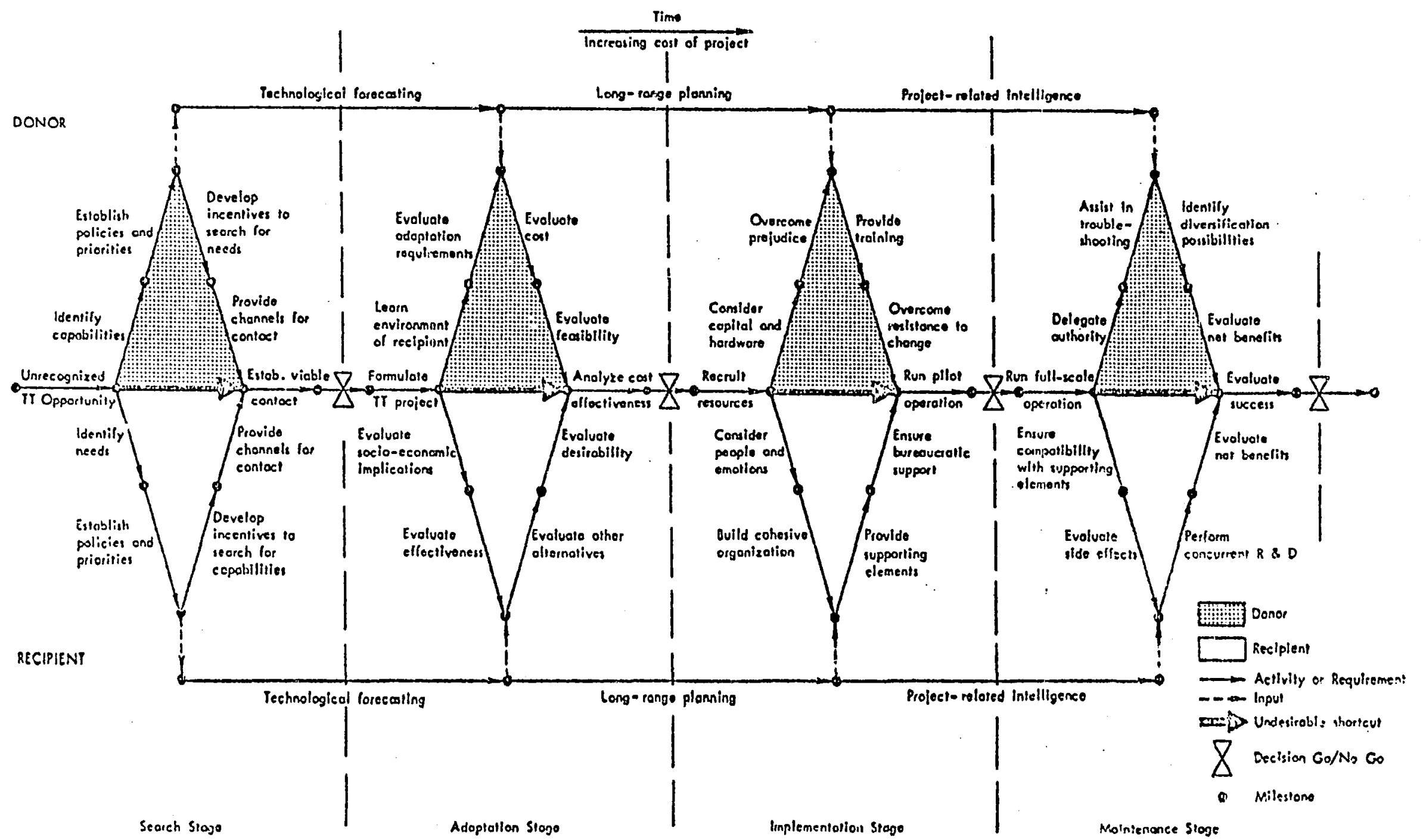

Figure 5. Technology transfer model (network type) 
(a) all activities above the center line are carried out by donor.

(b) all activities below the center line are carried out by recipient.

(c) all activities on the center line are carried out by both the donor and the recipient.

(d) model is divided into four stages: Search, Adaptation, Implementation, and Maintenance

(e) there are four major decision points; at each stage, go/no go decisions are made based on criteria devised for each stage.

(f) costs of making a wrong decision increase as one goes further dowin the diagram.

(g) three broad activities are indicated. These are: forecasting, long-range planning and intelligence gathering related to the project. All three activities are independent but essential and must be performed by both donor and recipient.

(h) the nature of activities are mentioned in Figure 5 . In the Search stage, an important feature is the awareness of the opportunities and capabilities. In the Adaptation stage, the TT project is formulated; donor learns about recipient's environment and the recipient evaluates his socio-economic environment, since he is best qualified to do so. In the 
Implementation stage, the required natural and human resources and needs for sophisticated versus nonsophisticated equipment are assessed. In the Maintenance stage, TT is in full operation; donor delegates authority to the recipient and provides services and assessment of original expectations and net benefits.

The model suggested by Bar-Zakay is a useful model of TT. It is descriptive but points out essential activities comprising any TT project. To this extent, it is a generalized model. The shortcomings of the model seem to be that: (a) it does not specify explicitly the variables underlying TT, (b) it does not mention social indicators and leaves the task of identification to the analyst, (c) it does not show the interaction of factors such as human and social. The model is likely to be more useful at the strategic level as it provides a generalized framework for considering the TT process.

In summary, all previous inquiries into TT utilize an incomplete structure of the process of TT. Furthermore, they usually avoid functionai aspects of TT. The interaction and feedback inherent in complex systems and processes are not considered. Many of the approaches utilize regression equations which fail to expose cause/effect relationships. When economic variables are included, social and human factors are ignored and vice versa. Furthermore, the planning and policymaking aspects are not considered explicitly and hardly anything is said about the manner in which the outputs of the inquiries can lead to better planning 
and policymaking, and hence to system improvement. Together the approaches point out the need for a multidimensional, unified frame-work for understanding and analysing TT. Needless to say, these inquiries have significantly increased our understanding of TT process. With these comments in mind, attention will be shifted now to outline a unified, integrative approach, or a new inquiring system for TT. 


\section{CHAPTER III}

\section{THE PROPOSED INQUIRING SYSTEM: INTEGRATIVE, UNIFIED APPROACH TO TECHNOLOGY TRANSFER}

The previous two chapters outlined the TT (Technology Transfer) pioblem and indicated the lack of an integrative, unified approach to TT. It was also pointed out that the previous inquiries fail to answer adequately the generating questions considered important for planning and policymaking purposes.

The approach to T T proposed in this thesis is derived by following a systematic procedure beginning by establishing objectives for developing the methodology. Having specified the objectives, requirements necessary to realize them are generated. Finaily, the proposed inquiring system $^{2}$ for $\mathrm{TT}$ is described and discussed within the framework of the established objectives.

The nature of the proposed inquiry is holistic. The spirit in which the IS is conceived is a mix of Kantian, Hegelian, and Singerian. It is Kantian in the sense that both model and data are considered together

2. Inquiring System will be abbreviated IS in the rest of this discussion. 
rather than separately. It is Hegelian since opposing viewpoints are and can be allowed in the IS; for instance, the consideration of the consequences of $\mathrm{TT}$ is clearly a Hegelian issue. The holistic nature of the IS, and the consideration that the system designer is a fundamental part of the system, makes it Singerian. Since the Singerian IS is a meta system containing all other IS, it can be said that the attempt here is to be Singerian. However, it seems useful to draw the distinctions in terms of Kantian and Hegelian, as mentioned above.

\subsection{Objectives for Developing the Methodology}

The derivation of objectives is usually not an easy task. Hence a discussion of relevant bases for establishing objectives is provided.

For our purpose, a representation of $\mathrm{TT}$, different from the previous approaches, is needed. A unified, integrative treatment of TT requires considering the aspects relevant to TT. The major shortcoming of the previous approaches is the inadequate representation of dimensions critical to TT. A narrow view is dangerous because it obtains simplicity at the expense of systems structure. On the other hand, a broad view of the system normally increases complexity.

One us eful way to reduce complexity and obtain simplicity is suggested by Ashby $[8,9]$. Ashby's law of requisite variety suggests that system complexity can be alleviated by reducing the variety exhibited by the system and/or by increasing the variety available to the analyst. Therefore, the question is not "what are the variables?", but "what variables are relevant and significant for the problem at hand?". 
(Forrester also points out this simple fact in all his studies.) For a particular situation, the usefulness of the law of requisite variety can be assessed by comparing the loss of information due to simplification with the gains from reduced system complexity.

The scope and nature of any TT problem can be represented in a unified but simple way as shown in Figure 6. Thus, a technology can be transferred horizontally or vertically within a country $A$ or from a country A to a country B. Each TT involves the problem solving procedure consisting of the following four major steps: evaluation including forecasting, planning, implementation and evaluation or the assessment of the impact or consequences of the impact or consequences of the transferred technology. Alternatively, the steps could be those suggested by Ha11 [36]: problem definition, value system design, systems synthesis, systems analysis, optimization of alternatives, decisionmaking and planning for action. The transfer can take place horizontally or vertically from any verticai level. The country may choose to transfer the technology at either an "early" or "late" stage of development; the term "early" implies that transfer occurs before the technology is fully established or is in the production phase in the donor country. For example, Japan chose to develop many transistorized devices (e.g., transistor radio) even beiore the corresponding technology was well-established in the U.S.A., thus capturing important markets. The term "late" implies that the country borrows a technology after it is well developed in the donor country; for instance, the transfer of U.S. computer technology to Europe. The terms 


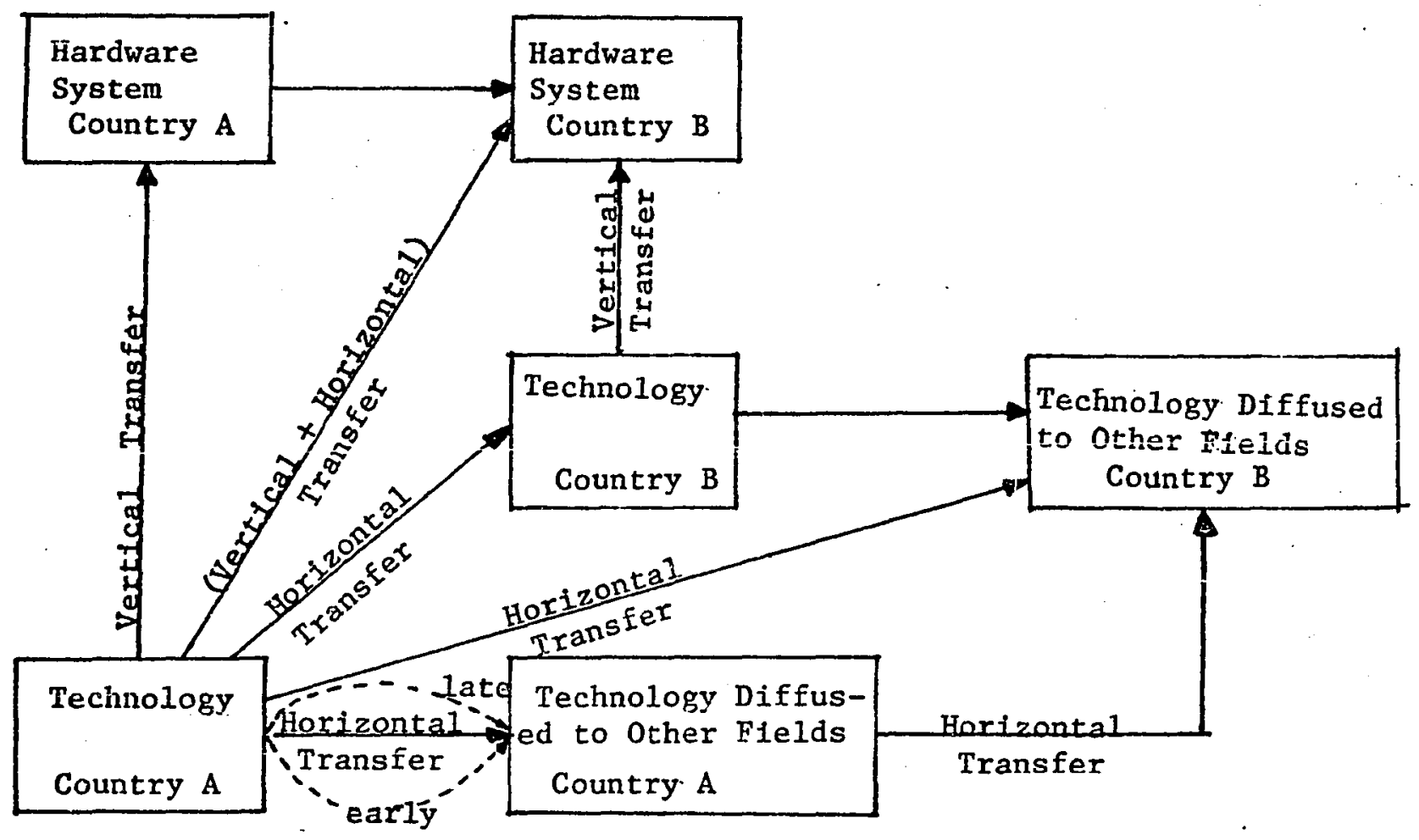

Each Arrow indicates the following process:

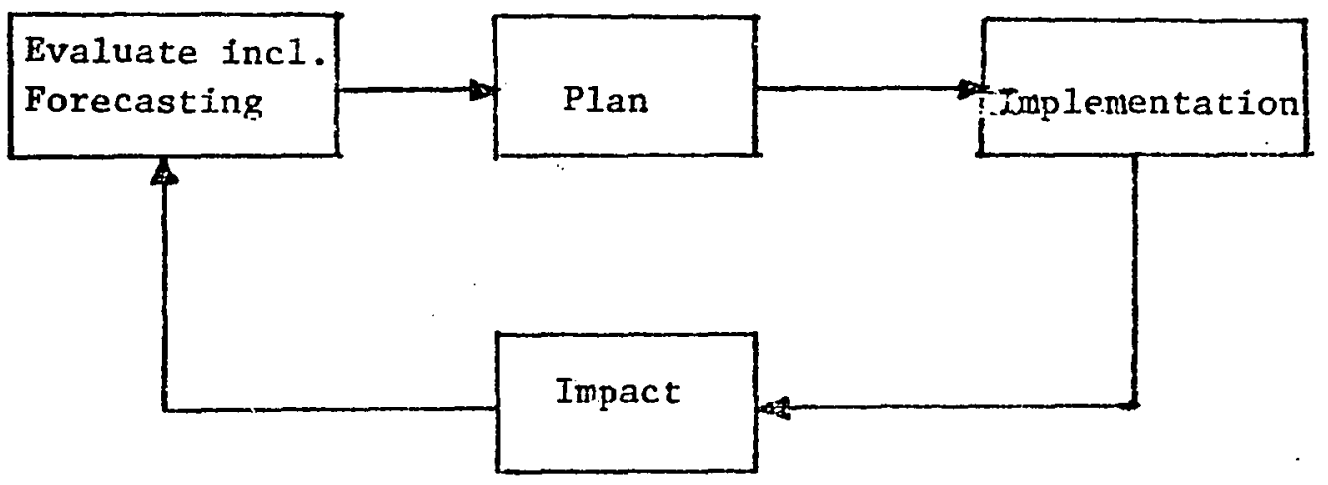

Figure 6. Technology transfer: relational concepts 
early and late bring in the time dimension of TT problem. The representation in Figure 6 depicts one systemic and unified view of the TT process.

The following objectives for developing the proposed approach are specified, based on the representation shown in Figure 6, and considering the complexity aspects:

1. To develop an integrative and unified approach to study the TI proilem, emphasizing the desirability to answer the important generating questions mentioned in Chapter I. The approach should be integrative in the sense that a multidimensional viewpoint is maintained; it should be unified in the sense that it considers the TT problem whether its scope is intercompany, interregional or international.

2. To develop a structure general enough to be useful for any TT problem. As mentioned earlier, the structure consists of significant variables together with their relationships.

3. To develop a framework for analysing and designing TT, using the structure developed.

4. To emphasize planning and policymaking aspects related to $\mathrm{TT}$.

5. To illustrate the usefulness of the approach by considering a TT from a developed to a develuping region.

3.2 Requirements to Realize the Objectives

The requirements are statements expressing what must be done to accomplish the established objectives. Ideally, some criterion must be 
devised to test the feasibility or realizability of the stated objectives. It seems necessary to meet the following requirements so that the stated objectives can be realized:

1. From the review of previous work and a survey of available measures for $\mathrm{TT}$, it seems that a purely quantitative approach to the TT problem is not possible and may not be even desirable. The principal reasons for this statement are: (a) the non-existence of uniform social indicators, (b) the lack of suitable data banks useful in deriving empirical relationships, and (c) incomplete understanding of basic socioeconomic phenomena underlying regional and national development. Thus, most previous studies on $\mathrm{TT}$ deal with economic variables to facilitate quantification.

The prime requirement then is to develop a language useful in communicating the problems and the characteristics of TT. Koontz $[48, \mathrm{p} .10]$ suggests the term "management theory jungle" for the abundant literature on management and organization theory; much of this literature is of little help to the practioners [103, pp.252-253]. To some extent, the same criticism can be applied to the literature on TT. The proposed approach, therefore, must provide a framework for developing such a language for planners and policymakers concerned with TT. The framework can take the form of a model or an inquiring system.

2. The proposed approach must allow policy planners and policymakers to translate their objectives and goals for $\mathrm{T} T$ into strategic and operational planning. Of special importance is the interfacing of longrange and short-range goals. 
3. The variables and measures used must have operational significance.

4. It is necessary to generate criteria or measures useful in assessing tine perírmance aspects of $\mathrm{TT}$. In particular, means must be found to express feasibility, desirability and efficiency (or applicability of transfer mechanisms) of TT. The current literature on $\mathrm{TT}$ is deficient on this score.

\subsection{Assumptions}

The assumptions are means to simplify the process of obtaining a problem solution. Often, they provide conditions for bounding the problem solution within reasonable limits.

Here, we specify assumptions into three broad categories as related to: (a) environment within which the TT problem exists, (b) structure of the TT problem, and (c) users or clients of the proposed IS for TT. The assumptions concerning environment help identify the environmental variables. These variables are not under the control of system analyst or designer but do affect $\mathrm{TT}$ performance. This viewpoint is based on Churchman's concept of environment $[20, p p \cdot 36,37]$. Here, we assume that political variables are in the environment. Thus, they will not be included explicitly in the $T \bar{T}$ structure. However, ofiten it may be fruitful to assess the sensitivity of TT performance to the nature of these variables.

The major assumption concerning TT structure is that the structure is time invariant. This assumption implies that the specified 
variables and their interrelationships remain invariant over the time period for which the $\mathrm{TT}$ is being considered. Although techniques to generate dynamic structure for TT can be derived, it does not seem possible to utilize them here for two major reasons: (a) it increases complexity of the structure considerably and the costs/benefits of the increas ed complexity are difficult to assess, and (b) a sequential problem solving process is preferred; thus, the first step is to develop a convincing framework for looking at TT problems rather than adding complexity. In section 1.4 , the users were assumed to be planners and policymakers. The implications of this assumption were also discussed there. It can be recalled that the major implication dealt with the usefulness of the approach in terms of planning and policymaking requirements.

\subsection{The Proposed Framework of the IS for TT}

The proposed framework is based on the premise that any TT can be studied in terms of the dimensions critical to it. The system or process structure can then be developed utilizing these dimensions. Basicaliy, there are three dimensions critical to any TT. These are: Resources, Transfer Mechanisms, and Impact or Consequences of TT.

Resources are the means that a TT uses to do its jobs. Resources are the general reservoir out of which the specific actions of a $T T$ can be shaped. Resource requirements and availability determine if a TT is feasible. Technological forecasting can be useful here to estimate the occurrence of likely technical advances which can improve or increase resource productivity. The identification of real resources for $\mathrm{T} T$ under 
investigation is a critical dimension that must be considered. Resources can be further categorized as human resources, natural resources, etc. Mechanisms or channels of transfer are utilized to transfer a technology. The characteristics of such channels can affect significantly the outcomes of TT. For instance, the Government of India chose itself as the mechanism to transfer steel technology from Britain, Germany, and U.S.S.R. to India. The outcomes were quite different in terms of financial burdens and production outputs when compared to previous trans fers of the same technology from U.S. to India, but utilizing business as the transfer mechanism. The channels of TT usually tend to overlap, but a few distinct channels exist. Thus, it is important to consider TT along this dimension. The major intent is to determine the applicability of various mechanisms to the $\mathrm{TT}$ under investigation.

Much has been written on the subject of the impact of technology. In the long run, the consequences of technology are difficult to predict, although they do affect the environment, especiall $y$ that of the recipient region or country. For instance, the long-run consequences of transferring to India a technology to manufacture small, economic automobiles can be significant in terms of its impact on the social outlook, modes of transportation, environmental damage, to name a few. Thus, although most previous studies on $\mathrm{TT}$ consider the consequences of $\mathrm{TT}$ as an exogeneous dimension, it seems wise to include it in the TT structure to be developed here. This dimension is utilized to assess desirability of a $\mathrm{TT}$ in terms of its impact on the recipient's sociocultural and economic environment. 


\subsection{A General, Basic Model of the IS for TT}

The development of the proposed IS for TT can be explained in terms of the model of Figure 7. The inputs -. objectives for TT and the technology to be transferred -- are given. The outputs can be generated using these inputs and specified decision criteria. Such outputs can be: TT policies prescribed in terms of resource requirements, applicable mechanisms and impact on technological and non-technological environment. The returns are the following performance measures: feasibility of $\mathrm{TT}$ in terms of the resources required, efficiency of $\mathrm{TT}$ measured in terms of applicability of transfer mechanisms and desirability of the consequences of $T T$. The effectiveness of $T T$ can then be measured as a function of these three performance meăsü outputs and performance helps in the planning of the region's resources, determining the means to exploit the efficiency of useful transfer mechanisms and anticipating the consequences of the technology to be transferred. If utilized properiy, these inpuiz to planning and policymaking can help in reducing uncertainty associated witk TT. As Schon [82,p.110] points out, the transformation of uncertainty into risk is sometimes the best and the only thing a planner can do.

Utilizing the three critical dimensions considered earlier and the basic model of Figure 7, a hierarchical structure can be developed to understand, analyse and design a TT. 


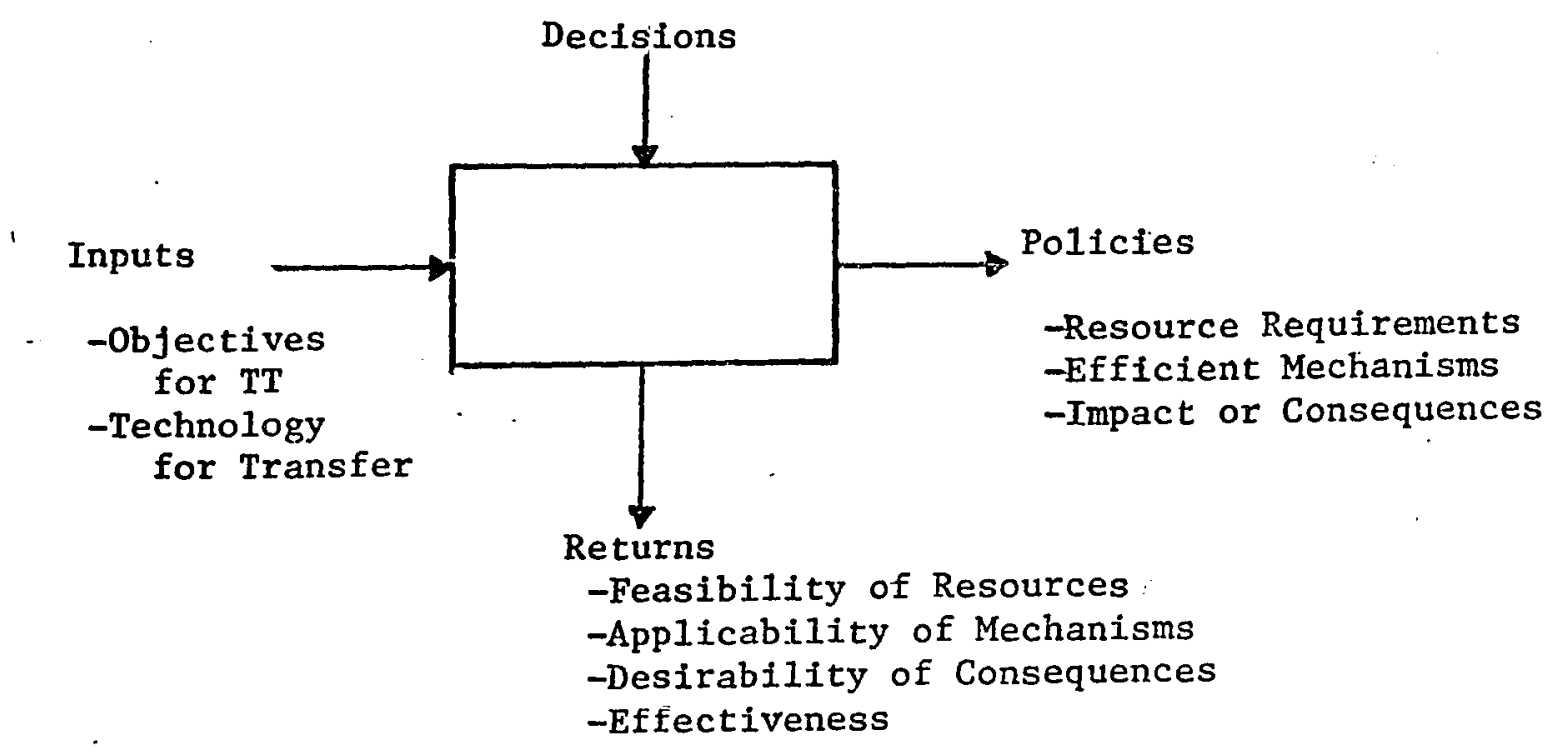

Figure 7. Basic framework for developing the IS for TT 


\subsection{Development of the Hierarchical Structure}

The term hierarchical usually refers to the vertical arrangement of levels and dependence of higher levels upon the performance of lower levels. Simon in his classic paper [84] has set forth strong arguments for believing that hierarchic structure is the rule rather than the exception in physical, biological, social, symbolic and many other types of systems. When faced with a complex problem, it becomes a reasonable hypothesis that its structure is hierarchic.

It also seems useful to develop a hierarchical structure of the IS for TT. The hierarchical structure is attractive on the following grounds:

(a) it allows flexibility to the clients of the IS, since they can develop and utilize the hierarchical level best suited to their needs.

(b) it allows separation of units that have maximum informa tion flow among each other.

(c) it allows systemic treatment of the TT problem, i.e., one begins at the lowest level to formulate the static structure and then works upwards developing dynamic structure of TT.

(d) it represents more closely the sequential nature of decisionmaking so prevalent in human behavior. Thus, the decisionmaking begins at the lowest level of the structure proceeding to the top level. For instance, Cyert and March [24,p.118-119] suggest that decisionmaking and problem solving in organizations is sequential in nature.

During this study, it seemed fea sible to develop a hierarchical representation for the TT problem. The three critical dimensions -- 
resources, transfer mechanisms and consequences -- form the basic framework for developing each level. A three level hierarchical structure was developed as shown in Figure 8. Realizing that all clients of the IS may not be interested in studying the TT problem in its full scope and dynamism, the lowest level (Level 1) is developed in a check list form and provides a static structure of TT. Thus, Level 1 specifies variabies relevant to the thee critical dimensions, along with their associated measures. The resulting structure looks like a tree. Since the tree explicitly states all possible variables and their measures, it is labeled "Morphological Tree".

At the intermediate level (Level 2), the interactions between the significant variables, specified in Level 1 , are developed. The interactions represent relationships between the variables. Thus, the complete structure of TT under study is specified in Level 2. The structure is useful in assessing outputs and returns discussed earlier and also for testing decision criteria and policy implications. Since interactions are developed here, Level 2 is labeled "Interaction".

The generating questions cited in Chapter I can be answered, for a particular TT, by developing Levels 1 and 2 . The ultimate object of the student of TT however is to be able to design the best or optimal TT to meet the objectives specified by policy makers and planners. Such a design procedure should evaluate systematically the available technologies, mechanisms and environments to produce the most satisfactory TT along the three critical dimensions. Ansoff [6] has suggested one 


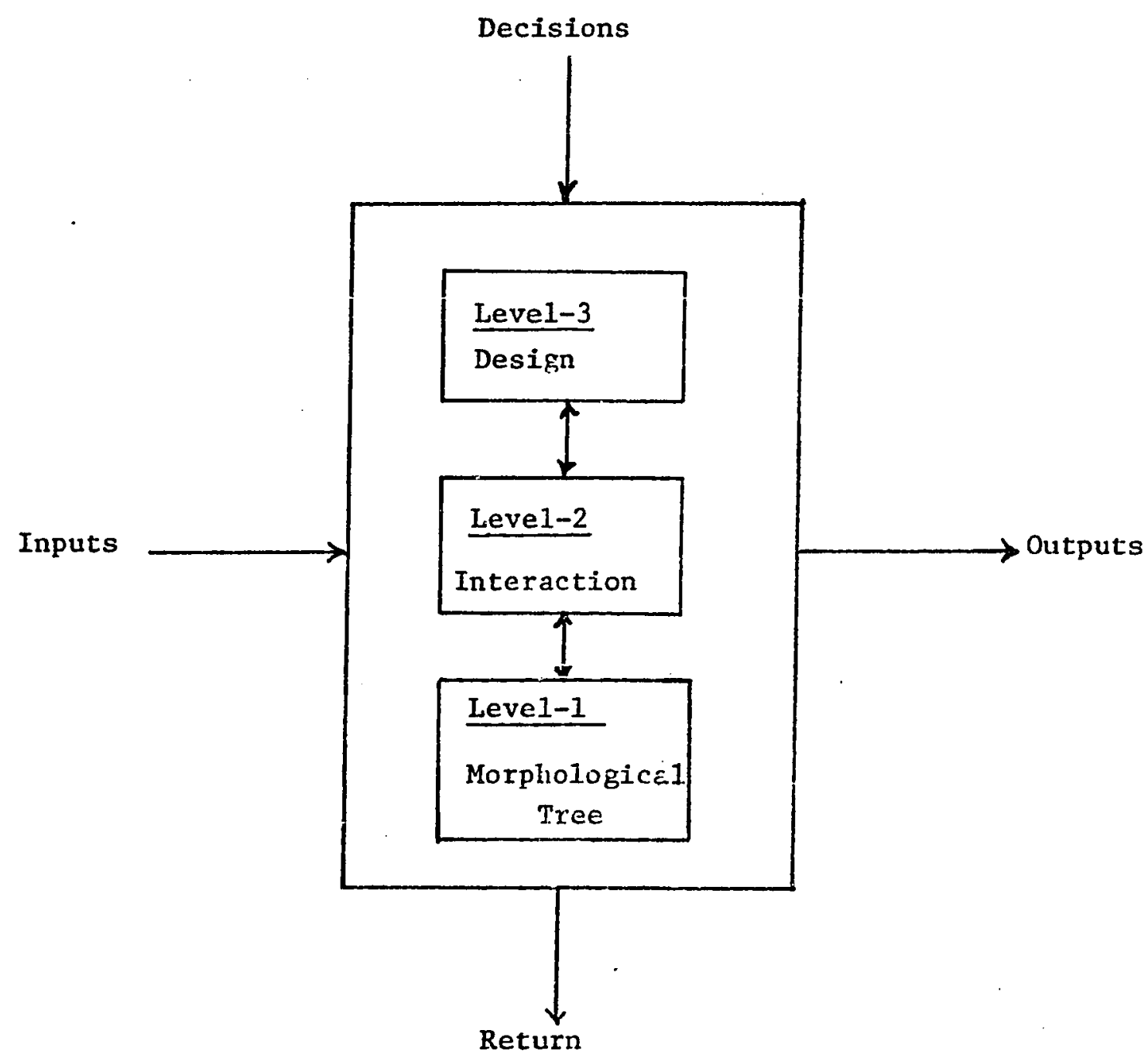

Figure 8. The proposed hierarchical structure of TT 
approach to organization design. His design approach can be extended here to levelop Juevel 3 which utilizes one such process for the design of TT. Because of the complexity involved and the lack of adequate data, Level 3 is utilized here only to provide a larger framework for developing Levels 1 and 2. Level 3 completes the proposed hierarchical structure for studying TT.

The relationships of the three levels for investigating a $\mathrm{TT}$ are shown in flowgraph form in Figure 9. Each of these levels will now be discussed in some detail emphasizing its development and usefulness.

\subsection{Level 1: Morphological Tree}

The term morphology is attributed usually to $Z$ wicky [105]. The morphological technique is an analytical scheme emphasizing structural differences and/or similarities rather than functional or performance features [10, chapter 5]. The term morphological tree is used here to indicate structural differences of TT along the three critical dimensions -resources, transfer mechanisms and consequences. The structural differences along these three dimensions provide a static structure which represents the significant variables but omits relationships between these variables; these relationships are developed in Level 2.

A generalized morphological tree was developed and is shown in Figure 10. The three branches of the morphological tree have been made to correspond to the three critical dimensions cited above. These branches are further divided into subbranches which specify subdimensions of the respective critical dimension. Each subdimension further branches down 


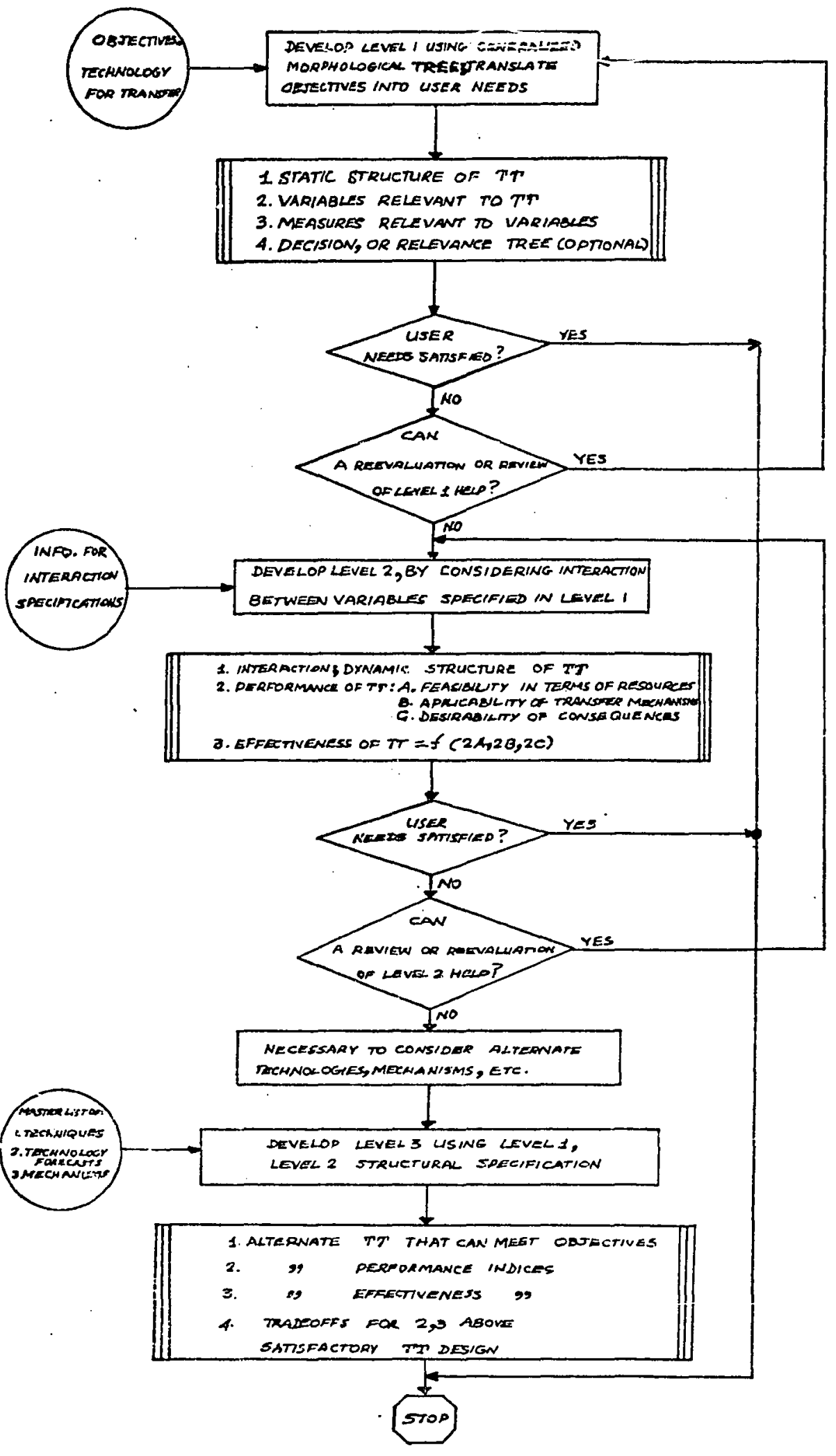

Figure 9. Simplified, overall flowgraph of the proposed method 


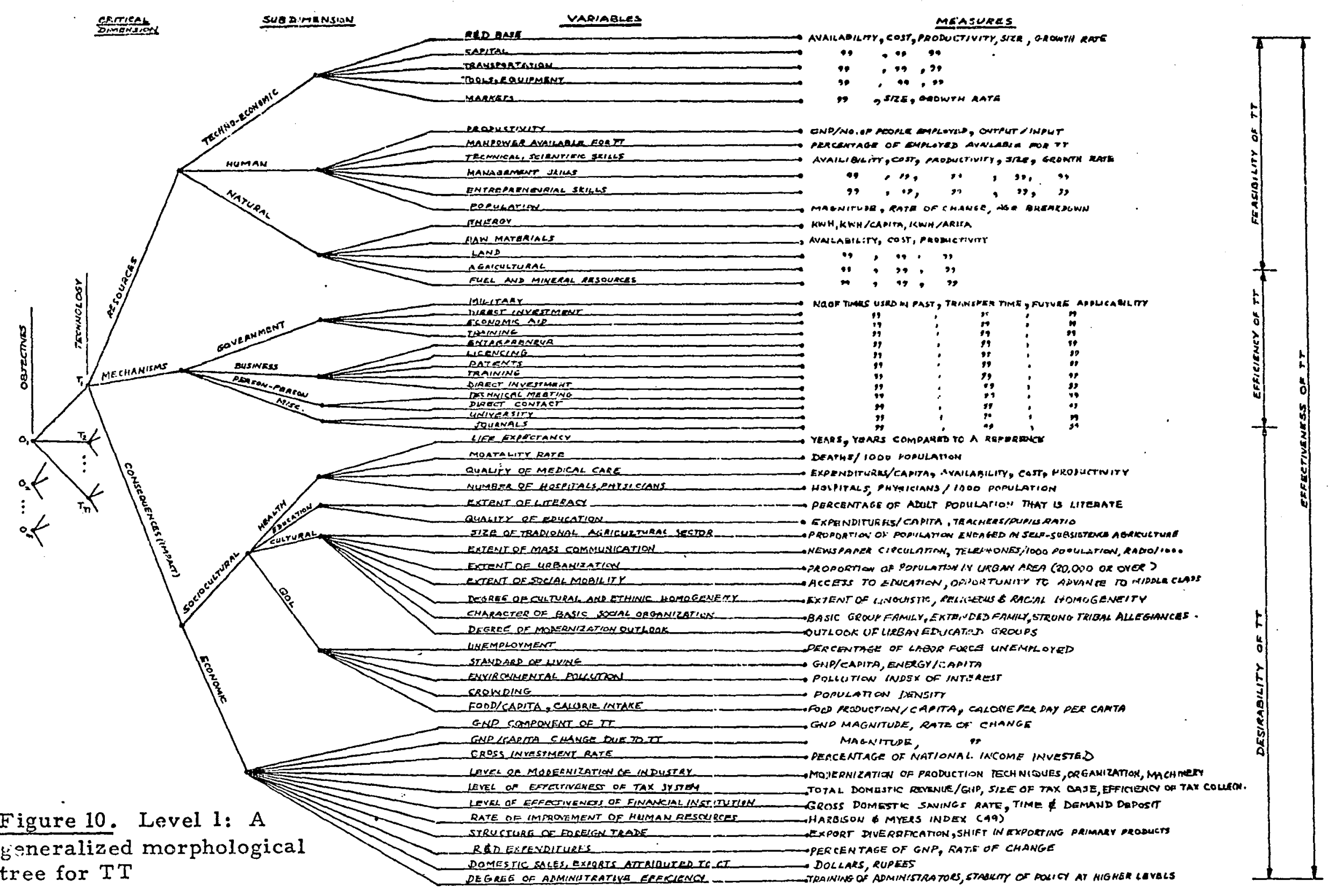


to variables relevant to that subdimension. Finally, measures useful for expressing each of the variables are indicated. Notice that some of the variables are measured as composite indices. Also, it can be noted that the structure in Figure 10 is general enough to be useful in investigating any TT whether its scope is intercompany, interregional or international.

The morphologicaí tree depicts the probiem structure by identifying relevant variables and their sequential evolution from basic informational inputs such as objectives for $\mathrm{T} T$ and/or selected characteristics of technology to be transferred. The resources are subdivided into three major categories: (a) techno-economic, (b) human and (c) natural. Each of these is further subdivided resulting into the specification of appropriate variables and measures for the resource dimension.

A variety of transfer mechanisms exists. These have been discussed by Brooks [17] and Spencer [89, Part III]. The transfer mechanisms are grouped into four major categories: (a) Government, (b) Business, (c) Person-Person, and (d) Miscellaneous, viz. University and Journals. Although these categories overlap somewhat, they can be treated separately [89, pp.33-37]. The measures for transfer mechanisms can be developed treating them as information channels.

The consequences (or the impact) of TT are grouped into two major categories: socio-cultural and economic. The socio-cultural category is further divided into: health, education, cultural and quality of life (QOL). Some of the QOL variables correspond to Forrester's work [31]. 
The writer is aware of the critism of Forrester's use of QOL term; however, for the lack of a better title, the QOL term is used. Each of the consequences or impact variable has an associated measure which is useful in characterizing it.

The following criteria were used to select the variables included in the morphological tree:

(a) its [variabie:s] useíliness in the TT probiem. This is established by considering the previous studies on TT. For example, the variables of human resources are discussed extensively in references 35 and 89.

(b) its demonstrated usefulness in past. Every variable in the morphological tree has been used explicitly (or implicitly) in the studies on social, economic, human resources, transfer mechanisms or cultural aspects of either TT or socioeconomic development. For example, many of the impact variables have been used in studies such as references 2,80 , and 91 .

(c) it must be measurable. The measure may be quantita tive or qualitative. In particular, references $89,2,80,92,34,68$, and 97 were consulted to determine the measurability oî ilie variabies.

(d) it should be useful in policymaking and planning. The major requirement here is that the variable should be controllable for policy and planning purposes.

The morphological tree can thus be visualized as a set of relevant variables. It is important that the elements of this set: (a) belong to a 
base set, (b) are of equal scope and significance, and (c) are reasonably independent of each other.

Level 1 provides a useful, general checklist to planners and policymakers. It explicitly identifies the variables which must be considered by the clients of IS for TT. From the generalized morphological tree, the client can derive a morphological tree applicable to his TT of interest. Thus, a particular TT under investigation acts as a "filter" for establish ing the relevant static structure. If the client so wishes he can add or delete branches from the tree of Figure 10. One cannot guarantee that the checklist is exhaustive but it is as complete as can be in terms of variable selection criteria described above. To the writer's knowledge, such a checklist for TT does not exist and is the first one to be developed. The morphological tree can also be used as a decision tree or a relevance tree. By specifying weights on each branch and subbranch, conventional decision or relevance analysis can be conducted. The weights have the same significance as they do in decision and relevance analysis. It seems convenient to indicate the performance measures of TT -- feasibility, efficiency and desirability -- on the morphological tree of Figure 10 by indicating how a particular performance measure applies to a particular dimension. Thus, the feasibility of TT can be measured in terms of resources required, efficiency of TT can be determined in terms of applicable transfer mechanisms, and desirability of TT can be expressed in terms of the consequences of TT. The effectiveness of TT can be defined as the degree to which the objectives (or goals) 
for TT are accomplished, and thus it is a function of the three per formance measures corresponding to the three critical dimensions. The procedure for developing levei $i$ is summarized in the flowgraph shown in Figure 11. The flowgraph is self-explanatory, considering the background discussion provided above.

The major outputs of Level 1 are: (a) static structure of the TT under investigation, and (b) measures for expressing the significant variables. These outputs become the inputs necessary to develo'p Level 2 .

\subsection{Level 2: Interaction}

In Level 1, variables relevant to $\mathrm{TT}$ under investigation are specified. In most practical situations, these variables interact with each other. For instance, a resource variable can affect another resource variable, or impact a sociomcultural variable. The former interaction can be termed self-interaction and the latter cross-interaction. At the component level, the possibilities of interaction are shown in Figure 12 .

\subsubsection{The Concept of Interaction}

The concept of interaction is the heart of the process to develop the structure of any system. An interaction can be expressed in various ways. A simple way to specify an interaction between two variables $x$ and $y$ is to state: $x$ affects or interacts with $y$, or $x$ does not affect 


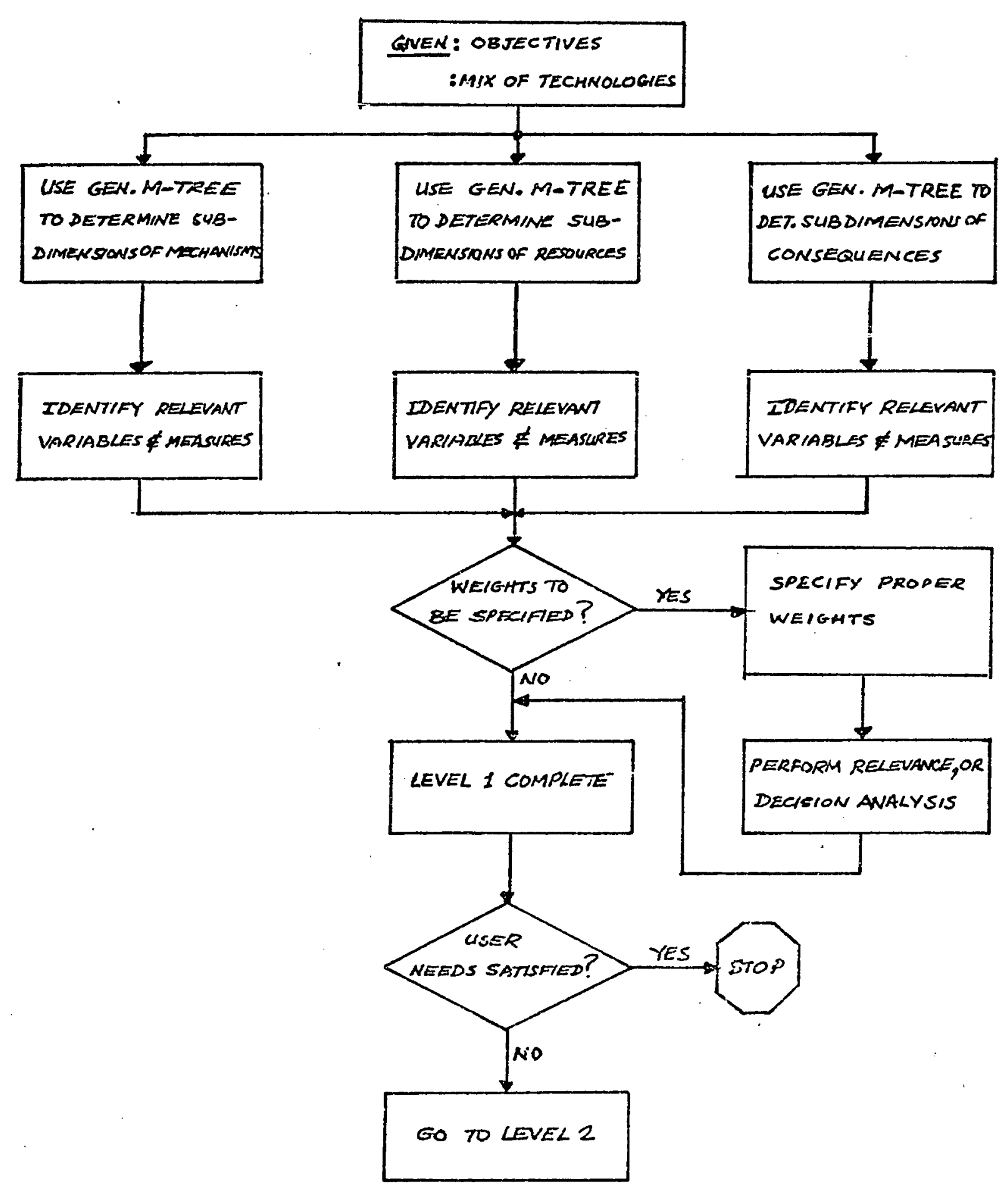

Figure 11. Flowgraph: Level 1 


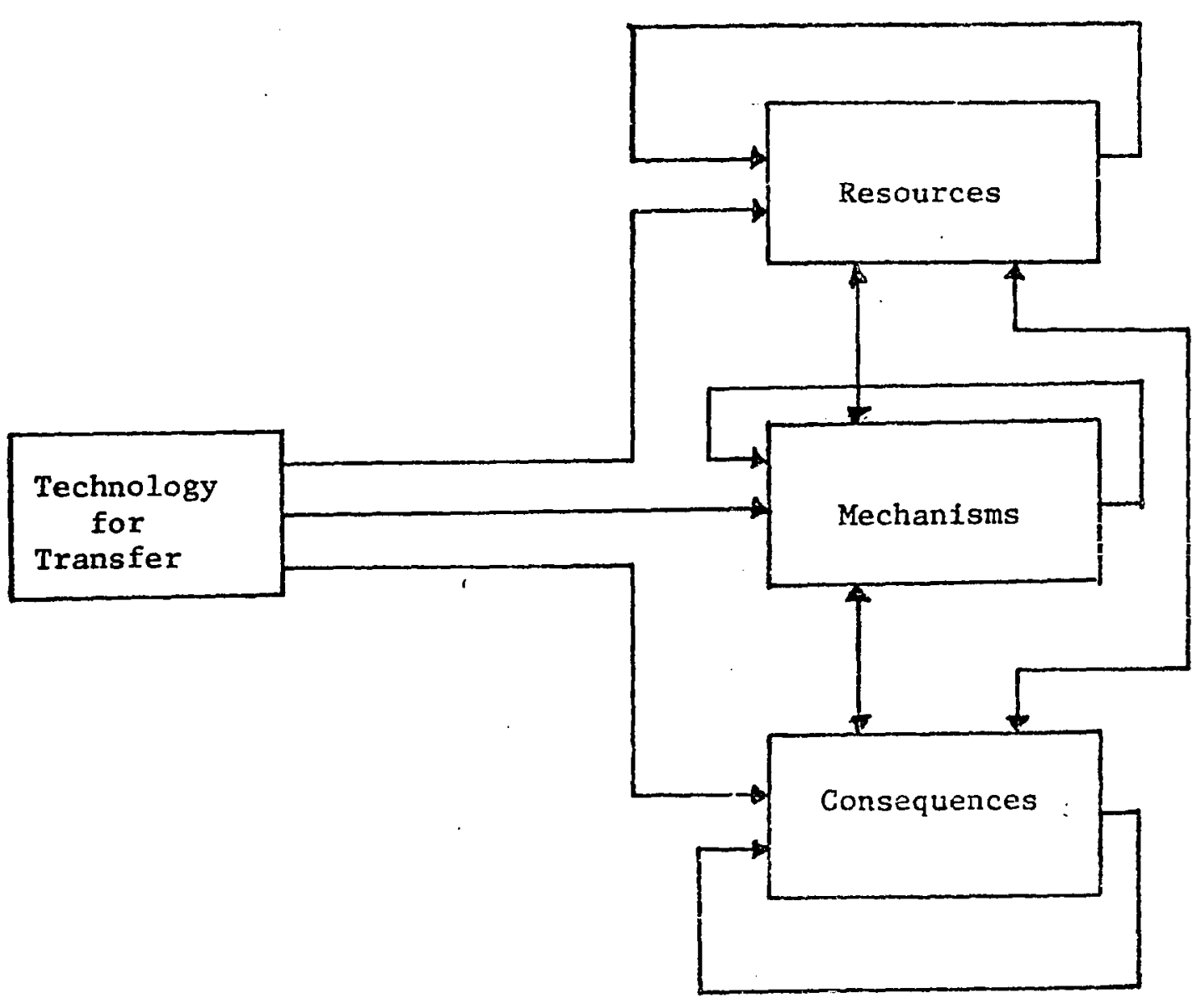

Figure 12. Possible interactions between technology, resources, mechanisms and consequences 
or interact with $y$. Symbolically, the two statements can be represented as: $x \rightarrow y$ and $x+y$. Alternatively, such an interaction can be called binary.

A continuous interaction can be specified as: $(x \rightarrow y: d)$ where $d$ represents the 'degree' or 'strength' of the interaction; $d$ can be a membership function [104], a relevance number [10,pp.171-174] or a number on a Likert scale [53]. The value of $d$ is normalized usually so that $0 \leq d \leq 1$, with 0 indicating no interaction while 1 indicates maximum interaction. For example, the interaction between $\mathbf{x}$ and $\mathrm{y}$ can be specified as: $(x \rightarrow y: d=0.8)$, implying that $x$ affects $y$ and the degree of interaction is 0.8 . Alternatively, $d$ may be thought of as the probability that interaction between $\mathrm{x}$ and $\mathrm{y}$ exists.

Finally, the interaction can be expressed as a functional relationship: $(x \rightarrow y: d), d=f(x, y)$, where $f$ is some mathematical function relating $d$ to $x$ and $y$. Surprisingly, the specification of interaction through mathematical functions is more common, although it is the most difficult way to express the interaction among variables.

All three specifications can be made to include time explicitly, if desired. In this case, the interaction between $x$ and $y$ can be expressed as follows:

(a) Binary: $(x \rightarrow y: d(t)), t=0,1, \ldots$; where $(x \rightarrow y: d=1$, or 0 ) is specified for discrete values of $t$; e.g. at $t=0, x \rightarrow y ;$ at $t=1, x+y$; etc. 
(b) Continuous: $(x \rightarrow y: d(t)), t$ can be discrete or continuous, but $0 \leq d(t) \leq 1$ must hold.

(c) Functional: $\quad(x \rightarrow y: d(t)), d(t)=f(x, y ; t)$

If the interaction is delayed, i.e., if $x$ changes, $y$ changes after a certain time interval $\Delta t$, the delay $\Delta t$ can be accounted for in the interaction specification as follows:

(a) Binary: $\quad d(t)-0$ for $t \leq \Delta t$, and equals 1 or 0 for $t>\Delta t$

(b) Continuous: $d(t)=0$ for $t \leq \Delta t$, and $0 \leq d(t) \leq 1$ for $t>\Delta t$

(c) Functional: $d(t)=f(x, y ; t+\Delta t)$, or $d(t)=f(x, y ; t, \Delta t)$

The mode of interaction, i.e., $x \rightarrow y$ positively or negatively, can also be specified. Often it is possible to define the variable and its associated measure in such a way that only the positive mode can occur.

The interaction $x \rightarrow y$, whether it is binary, continuous or functional, can be quantified or established in several ways. For complex systems, the interaction between system variables can be specified using the following techniques:

(i) intuitive techniques, e.g., Delphi, expert judgment.

(ii) statistical techniques, e.g., regression equations, correlation analysis using empirical data.

(iii) factor analysis.

(iv) information transmission concepts.

(v) Alexander's approach. 
The first technique is becoming increasingly popular. There are many books, papers and reports describing the use of Delphi [56]. The techniques (ii), (iii), and (iv) are useful when empirical or time series or statistical data are available. The firth technique is a combination of analytical, heuristic and intuitive techniques [3]. Since the first three techniques are well documented, only techniques (iv) and (v) are discussed here.

The use of a transmission function to specify relationships between variables has been popularized by Ashby [9]. The transmission function between the variables $\mathbf{x}$ and $y$ is defined as:

$$
T(x: y) \triangleq H(x)+H(y)-H(x, y)
$$

where $T(x: y)$ is a nonnegative measure of the strength of interaction between $x$ and $y$,

$$
\begin{gathered}
H(x)=\text { Entropy of } x=-\Sigma p_{i} \log p_{i} \\
H(y)=\text { Entropy of } y=-\Sigma p_{j} \log p_{j} \\
H(x, y)=\text { Entropy of the union of } x \text { and } y= \\
\quad-\Sigma p_{i j} \log p_{i j}
\end{gathered}
$$

The statistical data for $x_{i}$ and $y_{j}$, when available, are used to calculate the probabilities $p_{i}, p_{j}, p_{i j}$. These probabilities yield the values for $H(x), H(y)$ and $H(x, y)$ which in turn yield $T(x: y)$. By specifying a 
certain threshold, $T(x: y)$ can be transformed to a 0 or 1 specification for the binary case, while the value of $T(x: y)$ can be taker as the value of $\mathrm{d}$ for the continuous case. Some interesting applications of the above concept are demonstrated by Ashby [9] for reducing system complexity, and by Conant [22] for detecting subsystems of complex systems. Note that, to use this technique, probabilities must be calculated. Hence, the availability of a suitable data base is mandatory. Alternatively, the probabilities can be subjective, in which case the people familiar with TT under investigation should specify their values.

To explain Alexander's approach [3], the concept of a misfit variable has to be clarified. Before this can be done, it is necessary to explain the terms 'context' and 'form' as used by Alexander. The 'form' is the solution to the preblem; the 'context' defines the problem. Then, the misfit variable can be explained as follows $[3, p .101]$ :

"Any state of affairs in the ensemble which derives from the interaction between form and context, and causes stress in the ensemble, is a misfit".

Thus, any state of affairs which is somehow detrimental to the unity and the well-being of the whole ensemble is a misfit. Thus: "In architecture, for example, when the context is defined by a client, this client will tell you in no uncertain terms what he won't put up with." $[3, \mathrm{p} .101]$. The kettle (for boiling the water) which is uncomfortable to hold causes stress. Thus, the requirement "comfortable to hold" identifies one misfit variable. In most practical situations, the design which is the process of fitting form with the context involves eliminating the 
misfit variables rather than the vice-versa. This probably is the most subtle output of Alexander's work; that is, minimize (or eliminate) the number of "mis -fit" variables rather than maximize the number of "fit" variables.

Now, if one defines the probability of misfit occurring as $p\left(x_{i}=1\right)$ and that of avoiding the misfit as $p\left(x_{i}=0\right)$, the correlation coefficients for the two variables $x$ and $y$ can be defined as $[3, p .105]$ :

$$
C_{i j}=\frac{p\left(x_{i}=0, y_{j}=0\right) \cdot p\left(x_{i}=1, y_{j}=1\right)-p_{i}\left(x_{i}=0, y_{j}=1\right) \cdot p\left(x_{i}=1, y_{j}=0\right)}{\left[p\left(x_{i}=0\right) p\left(y_{j}=0\right) p\left(x_{i}=1\right) p\left(y_{j}=1\right)\right]^{1 / 2}}
$$

where $x_{i}$ and $y_{j}$ are the values that $x$ and $y$ take as a function of time or some other parameter. If $C_{i j}$ is markedly less than $0, x_{i}$ and $y_{j}$ conflict. If $C_{i j}$ is markedly greater than $0, x_{i}$ and $y_{j}$ concur, and if $C_{i j}=0$ then there is no noticeable interaction between $x_{i}$ and $y_{j}: \quad C_{i j}$ can again be used to specify the interaction as binary or continuous, as explained in the previous case. Again, the negativity can be a voided by appropriately defining a variable and its associated measure, although this may not always be possible. Alexander's notion of misfit variables will be often referred to in this dissertation. The previous comments regarding the availability of a data base and the use of subjective probabilities also applies here.

\subsubsection{Interaction Matrices: Structure of TT}

The interaction between variables can be depcited in a graph or a matrix form. The matrix form is more compact and will be utilized extensively here. 
There are two types of matrices: self-interaction and crossinteraction. Referring to Figure 12 (p.57), one can notice that there are three self-interaction matrices: $(R \times R),(M \times M)$ and $(C x C)$, where $R, M, C$ refer to the vectors of resources, mechanisms and consequences or impact variables. It also follows from Figure 12 that there are nine possible cross-interaction matrices: $(\mathrm{RxC}),(\mathrm{R} \times \mathrm{M}),(\mathrm{MxC}),(\mathrm{MxR})$, $(\mathrm{CxR}),(\mathrm{CxM}),(\mathrm{TxR}),(\mathrm{T} \times \mathrm{M})$, and $(\mathrm{T} x \mathrm{C})$, where $\mathrm{T}$ is a vector of technology attributes. All of these matrices are not of equal importance. In particular, the matrices $(\mathrm{RxM}),(\mathrm{MxC}),(\mathrm{MxR}),(\mathrm{CxR})$, and $(\mathrm{CxM})$ are of either second-order or little importance for the reasons outlined in the following paragraphs.

First of all, the matrices (CxR), (CxM) are of little interest because the impact of $C$ on $R$ and $M$ exists only in a long-run and at best can be treated as exogeneous relationships. The discounting phenomena, mentioned in section 1.4 , makes it difficult to quantify these relationships. Moreover, the relationships $R \rightarrow C$ and $M \rightarrow C$ precedes $C \rightarrow R$ and $\mathrm{C} \rightarrow \mathrm{M}$.

The matrix $(M \times R)$ specifies the relationships between transfer mechanisms and resources. The literature on $\mathrm{TT}$, such as reference 89 , does not support the hypothesis that the relationship $M \rightarrow R$ is significant. The non-existence of $M \rightarrow R$ relationship can be attributed to the fact that it is the technology that puts demands on resources. Whether a particular mechanism is employed or not is of little consequence to the fact that a particular resource is required. For instance, if technology 
to smelt iron ore is to be transferred, the basic requirement of iron ore as a resource does not depend on whether the government or a business concern acts as transfer mechanism. For similar reasons, the matrix (RXM) is of little interest also.

The matrix $(\mathrm{MxC})$ indicates the cross -interaction between trans fer mechanisms and consequences or impact variables. Again, the literature on $\mathrm{TT}$ and past transfers does not support the hypothesis that the relationship $M \rightarrow C$ is significant. The relationships $T \rightarrow C$ and $R \rightarrow C$ consider quite well the causes of impact on socio-cultural and economic variables. If a technology is introduced and necessary resources exist and are deployed for that technology, the transfer mechanisms can do little, if any, to change the nature and magnitude of the resulting consequences. For instance. the introduction of technology to manufacture relatively inexpensive, compact cars in India will impact the sociocultural variables, regardless of who -- government or business -undertakes to transfer the technology. On the other hand, the deployment of resources such as raw materials required for car manufacturing can significantly affect some of the economic variables.

Thus, we propose that the matrices $(T \times R),(T \times M),(T x C),(R \times R)$, $(\mathrm{MxM}),(\mathrm{CxC})$, and $(\mathrm{RxC})$ be considered as of prime importance. The matrices $(\mathrm{MxC})$ and $(\mathrm{MxR})$ are of second-order importance and can be considered for $\mathrm{TT}$ structure, if desired, to test the sensitivity of the results. The matrices, $(\mathrm{RxM}),(\mathrm{CxM})$, and $(\mathrm{CxR})$, are not of interest as the direction of interaction indicated by these matrices is opposite to that 
in the literature on $\mathrm{TT}$, and for the reasons cited above. The final structure ox TT, as proposed here, is shown in Figure 13. The dashed lines indicate the relationships of second-order importance.

The proposed structure, thus, consists of the three self-interaction matrices $(\mathrm{R} \times \mathrm{R}),(\mathrm{MxM})$ and $(\mathrm{CxC})$, and the four cross-interaction matrices (TxR), ( $T x M),(T x C)$, and $(R x C)$. Each entry in these matrices has a special significance and must be interpreted properly. In fact, the interaction cannot be specified until the significance of each entry is established. Table I explains the significance and the interpretation of each entry of the seven interaction matrices, for the three cases -- binary, continuous, and functional.

Alternatively, the structure of TT can be represented graphically. For this, let $\mathbf{r}_{i}, \mathrm{~m}_{j}$, and $c_{k}$ be the resources, mechanisms, and consequences or impact variables, and let $\ell_{r}, \ell_{m}, \ell_{c}$ indicate the links corresponding to all self and cross interactions of $r_{i}, m_{j}$, and $c_{k}$. Let $M$ denote the set of all variables $r_{i}, m_{j}$, and $c_{k}$ and $L$ denote the set of all links $\ell_{r}, l_{m}$, and $\ell_{c}$. Then, the graph $G(M, L)$ represents the structure of TT.

\subsubsection{Techniqiaes to Synthesize Interaction Matrices}

Our final intent is to be able to consider and describe all interactions -- direct and indirect -- of technology with resources, mechanisms and consequences. Hence, a technique is needed to synthesize the seven matrices into the following three matrices: $\left(T_{x R}\right)_{f},\left(T_{x} M\right)_{f}$, 


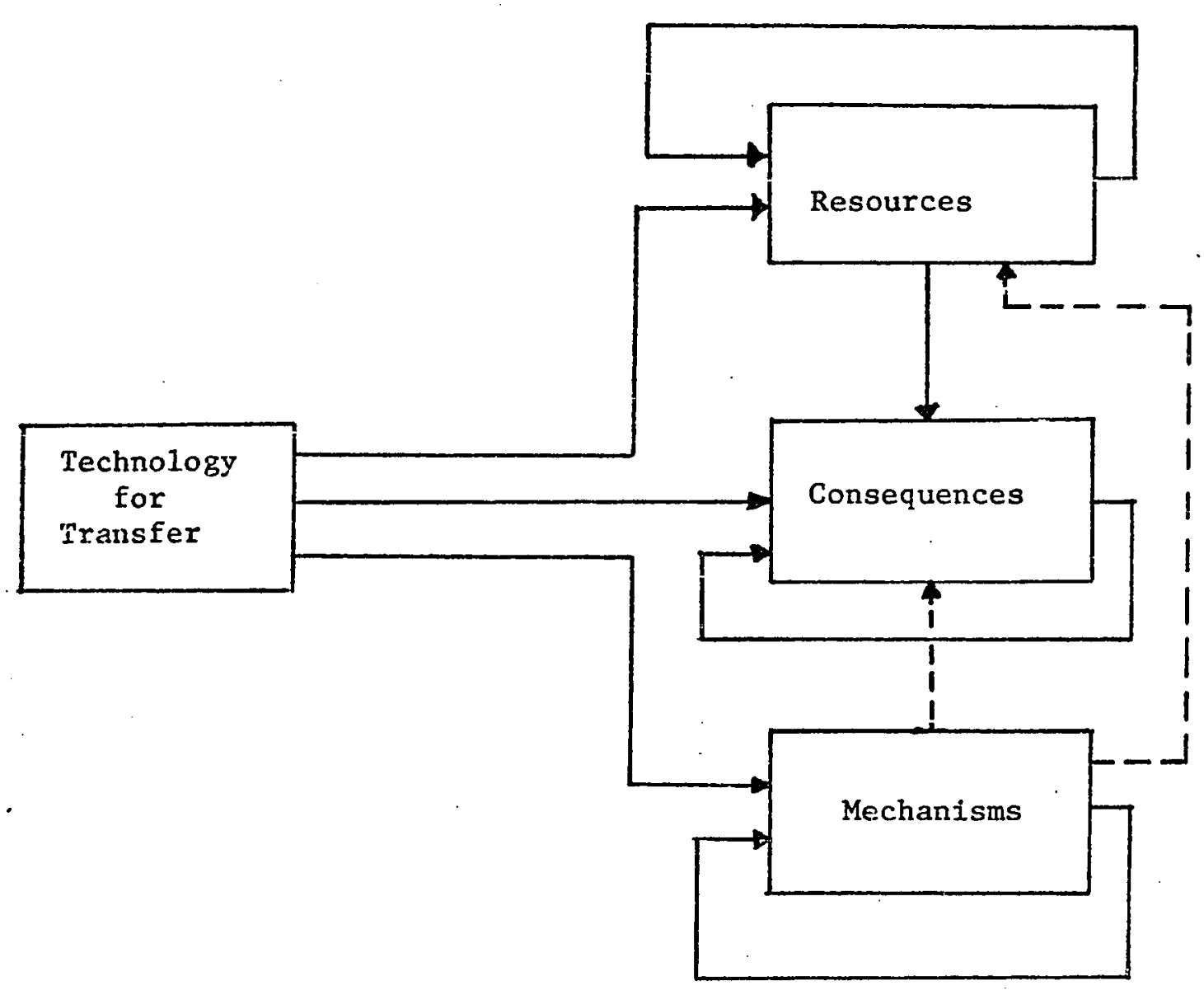

Figure 13. The proposed structure of TT 


\begin{tabular}{|c|c|c|c|c|}
\hline \multirow{2}{*}{$\begin{array}{l}\text { Relation }= \\
\text { ship }\end{array}$} & \multirow{2}{*}{ Interpretation or Significance } & \multicolumn{3}{|c|}{ Specification of the Interaction } \\
\hline & & Binary & Continuous & Functional \\
\hline $\mathrm{T}_{1} \rightarrow \mathrm{R}_{1}$ & $T_{1}$ requires $R_{1}$ & $\begin{aligned} T_{1} \rightarrow R_{1} & =1, \text { if } T_{1} \text { requires } \\
& =0, \text { otherwise }\end{aligned}$ & $\begin{array}{l}T_{1} \rightarrow R_{1}^{\prime}=d, \\
d \text { represents the } \\
\text { degree to which the } \\
\text { requirement exists. }\end{array}$ & $T_{1}=f\left(R_{1}\right)$ \\
\hline $\mathrm{T}_{1} \rightarrow \mathrm{M}_{1}$ & $\begin{array}{l}\mathrm{T}_{1} \text { can be transferred using } \\
\text { the mechanism } M_{1}\end{array}$ & $\begin{aligned} T_{1} \rightarrow M_{1} & =1, \text { if the mechanism } \\
& M_{1} \text { applies to } T_{1} \\
& =0, \text { otherwise }\end{aligned}$ & $\begin{array}{l}\mathrm{T}_{1} \rightarrow \mathrm{M}_{1}=\mathrm{d} \\
\mathrm{d} \text { representing de }- \\
\text { gree to which } \mathrm{M}_{1} \text { is } \\
\text { applicable }\end{array}$ & $T_{1}=f\left(M_{1}\right)$ \\
\hline $\mathrm{T}_{1} \rightarrow \mathrm{C}_{1}$ & 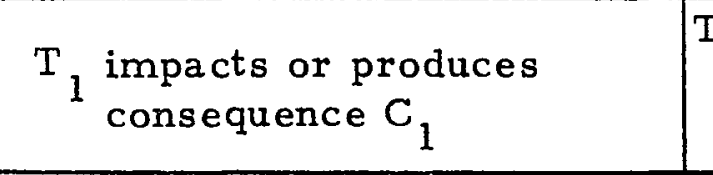 & $\begin{array}{c}T_{1} \rightarrow C_{1}=1, \text { if } T_{1} \text { impacts } C_{1} \\
=0, \text { otherwise }\end{array}$ & $\begin{array}{l}\mathrm{T}_{1} \rightarrow \mathrm{C}_{1}=\mathrm{d} \\
\mathrm{d} \text { representing de- } \\
\text { gree of impact }\end{array}$ & $T_{1}=f\left(C_{1}\right)$ \\
\hline $\mathrm{R}_{1} \rightarrow \mathrm{R}_{2}$ & $\begin{array}{l}\mathrm{R}_{1} \text { in turn requires } \mathrm{R}_{2} \\
\text { e.g. skills are required to } \\
\text { operate tools }\end{array}$ & Same as row & & \\
\hline$M_{1} \rightarrow M_{2}$ & $\begin{array}{l}\mathrm{M}_{1} \text { in turn can use } \mathrm{M}_{2} \text {, i.e. } \\
\text { the existence of an overlap, } \\
\text { e.g., military channel depends } \\
\text { on gov't direct investment. }\end{array}$ & Same as row & & \\
\hline $\mathrm{C}_{1} \rightarrow \mathrm{C}_{2}$ & $\begin{array}{l}\text { Impact on } C_{1} \text { results in an } \\
\text { impact on } C_{2} \text { e.g.,agricultural } \\
\text { sector size can affect food/capita }\end{array}$ & Same as row & & \\
\hline $\mathrm{R}_{1} \rightarrow \mathrm{C}_{1}$ & $\begin{array}{l}\text { Requirement of resource } \mathrm{R}_{1} \\
\text { would produce impact on } \mathrm{C}_{1} \text {, e.g. } \\
\text { labor productivity can affect } \\
\text { employment }\end{array}$ & Same as row & 3 & \\
\hline
\end{tabular}


and $(T \times C)_{f}$, where the subscript $f$ indicates that these are final or synthesized matrices containing direct and indirect relationships between $T, R, M$, and $C$.

It can be recalled that each element of the seven interaction matrices corresponds to direct link between the variables (since the interaction is specified between a pair of variables). The synthesis of of the seven matrices results into three matrices as indicated above. To illustraice thia, consider that $T_{1} \rightarrow R_{1}$ exists but $T_{1} \rightarrow R_{2}$ does not; also, assume that $R_{1} \rightarrow R_{2}$ exists. Then, $T_{1} \rightarrow R_{2}$ exists through the indirect path $T_{1} \rightarrow R_{1} \rightarrow R_{2}$. The relationships $T_{1} \rightarrow R_{1}$, and $R_{1} \rightarrow R_{2}$ would have been specified in the $(T \times R)$ and $(R \times R)$ matrices. Hence, a proper synthesis of ( $T x R$ ) and (RxR) matrices would yield the $(T \times R)_{f}$ matrix which will contain the $\mathrm{T}_{1} \rightarrow \mathrm{R}_{2}$ relationship also. This illustration considered an indirect path through the self-interaction matrix; cross-interaction matrices can also provide indirect paths from one variable to another. In the following discussion, we provide a technique to synthesize the seven matrices, for each of the three ways to specify interaction -binary, continuous and functional.

\section{Case 1: Binary Interaction}

We assume that the following seven matrices have been developed by the analyst: $(\mathrm{TxR}),(\mathrm{T} \times \mathrm{M}),(\mathrm{TxC}),(\mathrm{RxC}),(\mathrm{R} \times \mathrm{R}),(\mathrm{MxM}),(\mathrm{CxC})$. Any number of interaction matrices can be added, if they exist. Recall that the first four matrices contain cross-interactions, while the last 
three specify self-interactions. Indirect paths exist through both crossinteraction and self-interaction matrices. It is required to synthesize the seven matrices into $(T \times R)_{f},\left(T_{x M}\right)_{f}$, and $(T \times C)_{f}$ matrices which represent all interactions -- direct and indirect. For instance, $(\mathrm{TxC})_{\mathrm{f}}$ matrix must be developed by synthesizing $(T \times C),(C x C),(R \times R)$, and $(\mathrm{RxC})$ matrices. Such a synthesis would yield all direct and indirect paths from $T$ to $C$.

Denoting the logic or Boolean 'AND' operator by the symbol $\Lambda$ and the logic or Boolean 'OR' operator by the symbol $\mathrm{V}$, the indirect paths from $T$ and $R$ due to self-interaction ( $R \times R)$ can be expressed by the relationship:

$$
(T x R)_{s n}=(T x R) \Lambda(R x R)
$$

The matrix multiplication on the right-hand-side of equation (3.6) is performed as follows: the $i, j$ th element of the $(T \times R)_{3 n}$ matrix is equal to 1 , if any product of the elements of the ith row of (TxR) and the corresponding elements of the jth column of the (RxR) matrix equals 1 . For instance, if the ith row of ( $T \times R)$ is $(0,1)$, and the jth column of (RxR) is $(1,1)$, the $i$, jth element of $(T \times R)_{\operatorname{sn}}$ is 1 , since one of the two products in $(0,1) \times(1,1)$ equals 1 .

The direct and indirect paths can be synthesized by a logic 'OR' operation on $(\mathrm{TxR})$ and $(\mathrm{TxR})_{\mathrm{sn}}$. Thus:

$$
\begin{aligned}
(T \times R)_{s} & =(T \times R)_{s n} V(T \times R) \\
& =[(T x R) \Lambda(R \times R] V(T x R)
\end{aligned}
$$


The OR operation in equation (3.7) is carried out as follows: Compare the $i, j+h$ element of the $(T \times R)$ sn matrix to the $i, j$ th element of the (TxR) matrix, if both elements are equal to $O$, set the $i, j$ th element of the $(T \times R)_{s}$ matrix to $O$; otherwise, set the $(T \times R)_{s}$ element to 1 .

A simple example will illustrate the above concept. Consider;
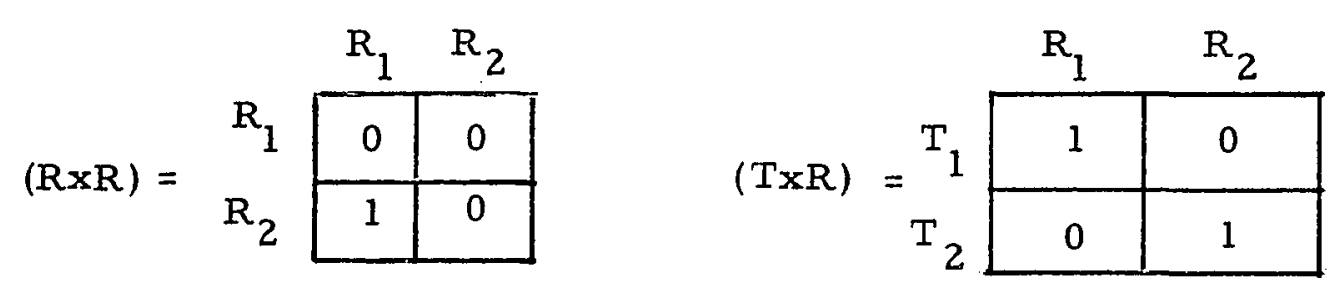

The above matrices are equivalent to flowgraph (a) below:
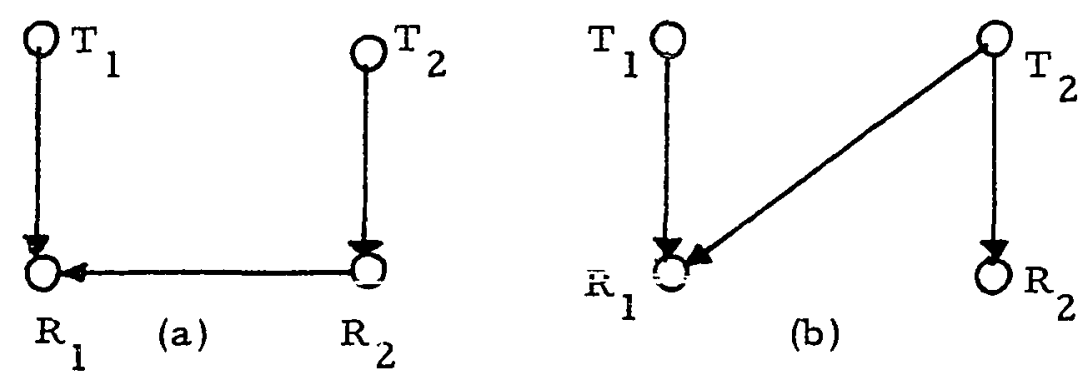

(b)

Synthesis of the direct paths $T_{1} \rightarrow R_{1}, T_{2} \rightarrow R_{2}$ and $R_{2} \rightarrow R_{1}$ shown in (a) would yield the relationships in (b). The matrix (TxR) must then specify the relationships between $T$ and $R$ shown in (b). Use of equation 3.7 yields: 


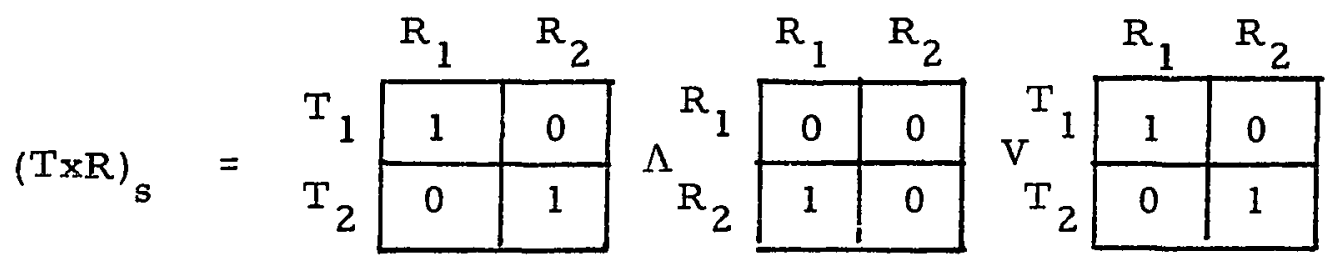

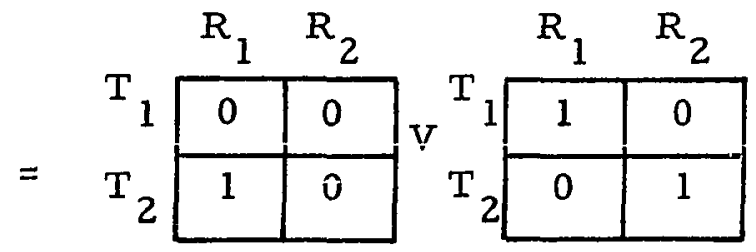

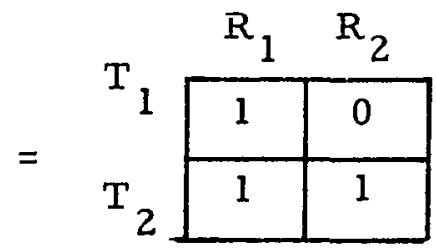

Notice that the (TxR) matrix checks out with the flowgraph (b) above. Extending the above technique to cover direct and indirect paths due to self-interaction, one gets:

$$
\begin{aligned}
& (\mathrm{TxR})_{\mathrm{S}}=[(\mathrm{T} \times \mathrm{R}) \Lambda(\mathrm{RxR})] \mathrm{V}(\mathrm{R \times R}) \\
& (\mathrm{T} \times \mathrm{M})_{\mathrm{S}}=[(\mathrm{T} \times \mathrm{M}) \Lambda(\mathrm{MxM})] \mathrm{V}(\mathrm{TxM}) \\
& (\mathrm{TxC})_{\mathrm{S}}=[(\mathrm{T} \times \mathrm{C}) \Lambda(\mathrm{CxC})] \mathrm{V}(\mathrm{TxC}) \\
& (\mathrm{RxC})_{s}=[(\mathrm{T} \times \mathrm{C}) \Lambda(\mathrm{CxC})] \mathrm{V}[(\mathrm{RxR}) \Lambda(\mathrm{RxC})] \mathrm{V}(\mathrm{RxC})
\end{aligned}
$$

Notice that $(\mathrm{R} \times \mathrm{C})_{\mathrm{s}}$ is developed by determining indirect paths through the two relevant self-interaction matrices $(\mathrm{RxR})$ and $(\mathrm{CxC})$.

Similar accounting for indirect paths due to the cross-interaction matrix $(\mathrm{RxC})_{\mathrm{s}}$ yields:

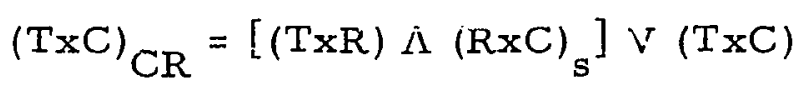


If matrices such as $(\mathrm{MxR})$ or $(\mathrm{MxC})$ exist, one would get similar expressions for $(\mathrm{TxR}) \mathrm{CM}$ and $(\mathrm{TxC}) \mathrm{CM}^{\cdot}$

Combining the direct and indirect paths due to self and cross interaction, we get the required synthesized matrices:

$$
\begin{aligned}
& (T \times R)_{f}=(T \times R)_{s} \\
& (T \times M)_{f}=(T \times M)_{s} \\
& (T \times C)_{f}=(T \times C)_{s} v(T \times C)_{C R}
\end{aligned}
$$

It can be noted that $(T x R)_{f}$ contains the direct paths specified by ( $T x R$ ) and indirect paths through $(R \times R)$. Similarly for the other matrices. It can also be noticed that essentially the above technique translates a mental model into a mathematically logical model.

The above procedure can be easily implemented on a computer. If the computer is unavailable, the matrices can be synthesized manually. This is not difficult for the binary case. The fact that the technique allows manipulation by hand is of particular importance in terms of the needs of developing countries who may lack computer facilities.

The matrices $\left(\mathrm{TxR}_{f},\left(\mathrm{TxM}_{\mathrm{f}}\right.\right.$, and $\left(\mathrm{T}_{\mathrm{x} C}\right)_{f}$ are useful in developing the three performance measures discussed earlier. For this purpose, we have ensured that all relationships _- direct and indirect -- are considered explicitly to analyse the performance of a $\mathrm{TT}$.

Appendix B contains a simple example which illustrates the above concepts and technique. 


\section{Case 2: Continuous Case (Interaction Specified on a $(0,1)$ Scale)}

Unlike the previous case, it is not possible to suggest a generalized synthesis technique for this case. The most useful approach is to evaluate the alternative synthesis techniques and select the one best suited for the given problem sitnation.

Consider the following simple case to illustrate the available aiternatives. Let $\left(x \rightarrow y: d_{1}=0.5\right)$ and $\left(y \rightarrow z: d_{2}=0.4\right)$. Then, the indirect interaction $x \rightarrow z$ can be specified as:

$$
\begin{aligned}
& \left(x \rightarrow z: \quad d_{3}=0.5 \times 0.4=0.2\right) \\
& \left(x \rightarrow z: \quad d_{3}=0.5+0.4=0.9\right) \\
& \left(x \rightarrow z: \quad d_{3}=\operatorname{Min}(0.5,0.4)=0.4\right) \\
& \left(x \rightarrow z: \quad d_{3}=\operatorname{Max}(0.5,0.4)=0.5\right)
\end{aligned}
$$

These are the basic propositions. Additional propositions can be generated by various combinations of the above four basic propositions.

The basic concern is to assign a value $d_{3}$ to the indirect path. Such an assignment is difficult mainly because we are uncertain of how the interactions propagate. In (A), we assume that the iwo interactions, $x \rightarrow y, y \rightarrow z$ are multiplicative. Such a multiplicative synthesis is justified, if $d_{1}, d_{2}$ are considered to be "system gain", as employed in linear systems. The signal flowgraph theory would then permit us to synthesize by multiplication.

In (B), the interactions are assumed to be additive. If $d_{1}, d_{2}$ represent 'utility', such a synthesis can be justified. The basic assumption underlying such a synthesis is the independence of $d_{1}$ and $d_{2}$. For 
instance, in relevance analysis, the basic assumption is that the utilities are additive. Notice that it may be necessary to normalize the result of addition.

In (C) and (D), decision criteria are employed. Since there is uncertainty about the way the interactions propagate, one can use a pessimist's criterion (i.e., C), or optimist criterion (i.e., D). Notice the possibility of using criterion such as minimax and maximin.

After careful consideration, it can be observed that of the four basic propositions, proposition (A) seems more logical for our purposes. The argument for this statement is as follows: when one attempts to take into account the indirect effects, the net relationship should diminish as the indirect path increases because the uncertainty is increasing. The multiplication of d's will always yield a number equal to or smaller than any of the numbers forming the product (provided $0 \leq d \leq 1$, which is already assumed). Thus, in (A), $d_{3}=0.2$ which is less than either $d_{1}$ or $\mathrm{d}_{2}$. In most practical situations, the above argument will usually hold. If the value $d$ of the indirect path $\left(\right.$ e.g., $\left.d_{3}\right)$ is calculated by multiplying the values $d$ of the direct paths (e.g., $d_{1}, d_{2}$ ), the synthesis technique for the binary case can be extended to this case also, by replacing the logic 'AND' by the arithmetic ' $A N D$ ' and maintaining the logic 'OR' operations.

One could argue that in some cases, the direct effects can cancel each other, thus making proposition (B) more attractive, For instance, if $\left(x \rightarrow y: d_{1}=1\right)$ and $\left(y \rightarrow z: d_{2}=-1\right)$, then the resulting indirect path 
$\left(x \rightarrow z: d_{3}=1-1=0\right)$. However, in the initial phases of design or problem solving, one presumes not to know the final state of the variables. Furthermore, if the variable and its measure is selected carefully, a situation where direct effects cancel each other will not arise.

In some cases, propositions $C$ or $D$ could be more suitable. For instance, since proposition $C$ selects the weakest link of the chain, it would be appropriate for a design which emphasizes the weakest component; proposition $D$, on the other hand, would be useful if the object is to maximize, e.g. profit.

Although we suggest the use of proposition (A), the ultimate choice is up to the designer or the inquirer.

Case 3: Functional Case: Interaction Specified as $(x \rightarrow y: d=f(x, y ; t))$

If interaction is specified through a functional relationship, again the four propositions mentioned in Case 2 (and their combinations) provide alternative techniques for synthesis. Most of the discussion for Case 2 holds for this case.

The concept of interaction matrix discussed above is similar to that suggested by Hill and Warfiald [37] and Gordon and Hayward [33]. However, there are some important differences. The Hill and Warfield approach requires that the variables or elements of the interaction matrix be arranged hierarchically (or in a 'tree' form), and that the selfinteraction matrix be symmetric. Gordon and Hayward also require reciprocity of the interaction matrix. In our case, the requirements of 
hierarchical arrangement and symmetry are relaxed. Therefore, even if $x+y$, the possibility of $y \rightarrow x$ is allowed. Furthermore, the propagation of interaction mentioned above is quite different from that suggested by Gordon and Hayward (e.g., their use of quadratic functional relationship). Also, Gordon and Hayward consider only the direct interactions. As mentioned above, we attempt to include all interactions - - direct and indirect.

\subsection{Level 3: Design}

Level 3 of the hierarchical structure is the most complex. The structure of Level 3 can best be explained through the flowgraph of Figure 14. The concern at Level 3 is to design an optimal TT using the information outputs of Levels 1 and 2, and master-lists of arailable technologies, mechanisms, environmental and technology forecasts. Conceputally, the suggested scheme in Figure 14 is similar to that suggested by Ansoff [6] for organization design. The flowgraph is selfexplanatory and needs no detailed elaboration. Notice, however, that a mix of quantitative and qualitative approaches is suggested for design purposes.

Level 3 utilizes information outputs from the two lower levels. The flowgraph depicts the necessary logical and decision steps involved in the design of a TT. Complexity of the problem, lack of proper data banks, and time constraints prevented a computer simulation of Level 3 . For this study, its usefulness is in checking and ascertaining that the $\mathrm{TT}$ under investigation meets the information, logic and decision 


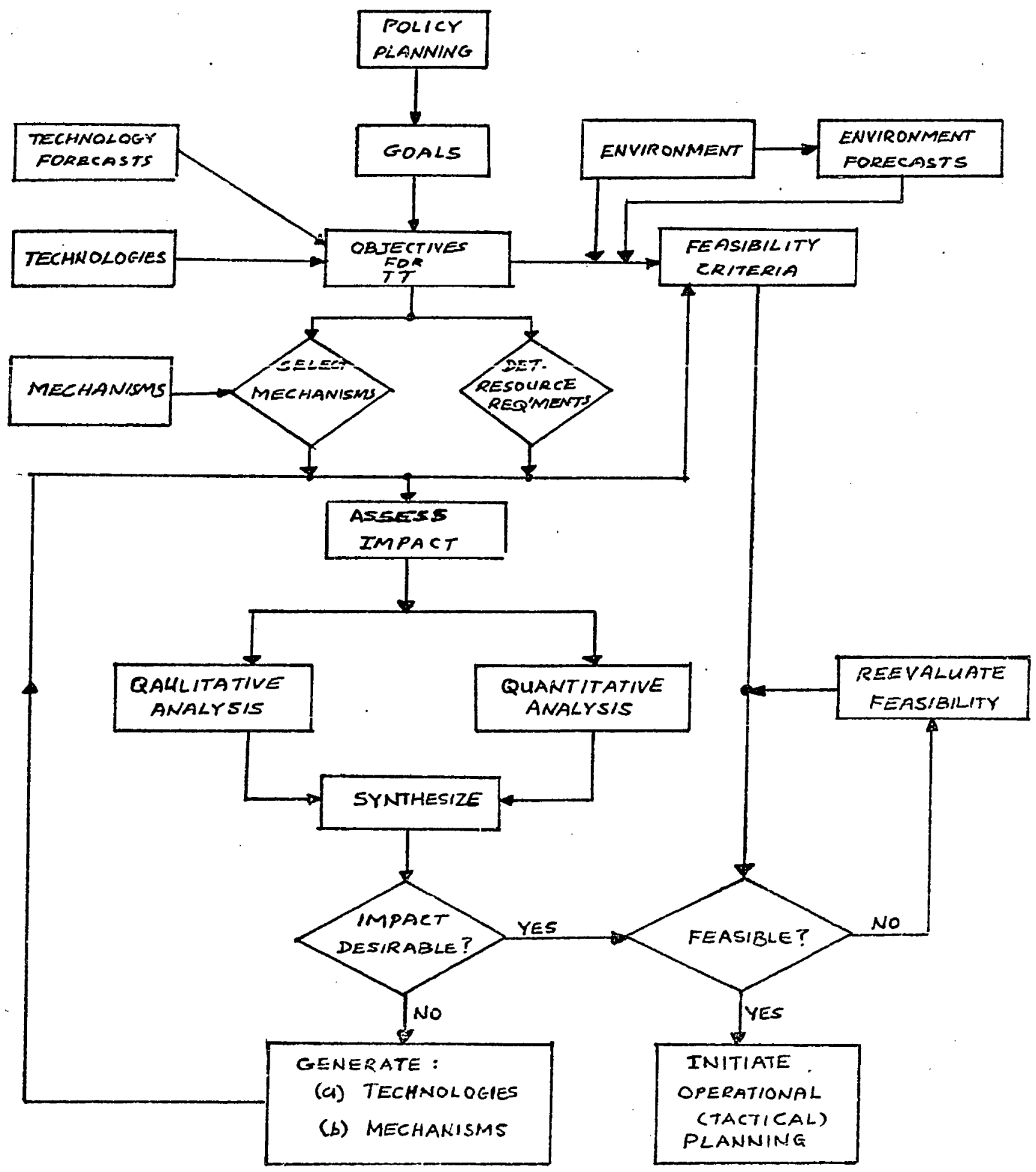

Figure 14. Level-3: Design process for technology transfer 
requirements specified in the flowgraph of Level 3. This is done by following manually each step of the flowgraph.

Such a design process, when properly implemented can produce

a TT design which will be optimal in terms of all three levels of planning - policy, strategic and operational.

\subsection{Performance Measures for TT}

It is possible to develop the three performance measures of TT -feasibility of $\mathrm{TT}$ in terms of resources required, efficiency of $\mathrm{TT}$ in terms of applicable transfer mechanisms, and desirability of consequences of TT -- using the outputs of Level 2, viz. the synthesized interaction matrices $(T \times R)_{f},(T \times M)_{f}$ and $(T x C)_{f}$.

\subsubsection{The Feasibility of TT in Terms of Resource Requirements}

The $(T x R)_{f}$ matrix specifies the resources needed for the various attributes of the technology for transfer. The technology attributes characterize the components of technology. For instance, computer technology can be characterized in terms of its two components: hardware and software; then, the rows of $(T x R)_{f}$ matrix specify the resources required for hardware and software components of the computer technology.

In order to determine the feasibility of $\mathrm{TT}$ in terms of resources needed, one can conduct the conventional cost/benefit analysis [ 74 , Chapters 4:-8]. At the policy level, such an analysis can be useful, if done properly --i.e., attempting to answer policy rather than 
operational planning questions. An alternative approach, which seems simpler than the conventional cost/benefit analysis, involves considering each resource variable in terms of its availability, opportunity cost and productivity (or quality). The analyst attempts to determine 'yes' or 'no' answers to the questions such as:

(a) is the resource available domestically? If not, can it be imported?

(b) is the opportunity cost of the resource reasonable? Does it seem profitable to deploy the resource for the TT in question? Is the cost high, when the resource is to be imported?

(c) what can we say about the productivity of the resource in question?

One can argue that answers to the above questions need a detailed analysis with respect to a variety of factors. Yet, in real life situations, policymakers, planners, politicians, and decisionmakers answer such questions in 'yes' or 'no' fashion, based on their mental models. If such is the case, we suggest translating these mental models into mathematically logical statements which can then be explored for policy and planning implications. Of course, if the answers can be based on detailed analysis, so much the better!

To formalize the above concepts, denote:

$a=$ domestic availability of the required resource,

$=1$, if the resource is available domestically

$=0$, otherwise 
$\mathrm{b}=$ productivity of the required resource

$=1$, if productivity is assessed as good or reasonable

$=0$, otherwise

$c=$ cost of deploying the resource for the TT in question

$=1$, if the cost is assessed as reasonable

$=0$, otherwise

The terms such as "reasonable" and "good" are to be defined in a context proper to the TT under investigation. Our aim here is to estimate the behavior of each variable at its extreme ( 0 or 1$)$ values, and upon examination of these results, to determine whether or not deeper explorations are indicated. For this investigation, the measures $a, b$ and c are assigned specific values appropriate for the TT under investigation. Using the above formalization, it is possible to prescribe the following policy statements for assessing the feasibility of transfer:

$$
\begin{aligned}
& \mathrm{P}_{1}: \quad \mathrm{TT} \text { is feasible iff (if and only if) } a \Lambda b \Lambda c=1 \\
& \mathrm{P}_{2}: \quad \text { TT is feasible iff } a \Lambda b=1 \\
& \mathrm{P}_{3}: \quad \text { TT is feasible iff } a \Lambda c=1 \\
& \mathrm{P}_{4}: \quad \text { TT is feasible iff } b \Lambda c=1 \\
& \mathrm{P}_{5}: \quad \text { TT is feasible iff } a \mathrm{VbVc}=1
\end{aligned}
$$

The verbal interpretation of, say $P_{1}$, is: the policy statement $P_{1}$ requires that TT is feasible if and only if the required resource is available domestically, and its productivity is good, and its cost is reasonable. These policies can be represented graphically as shown in Figure 15. 

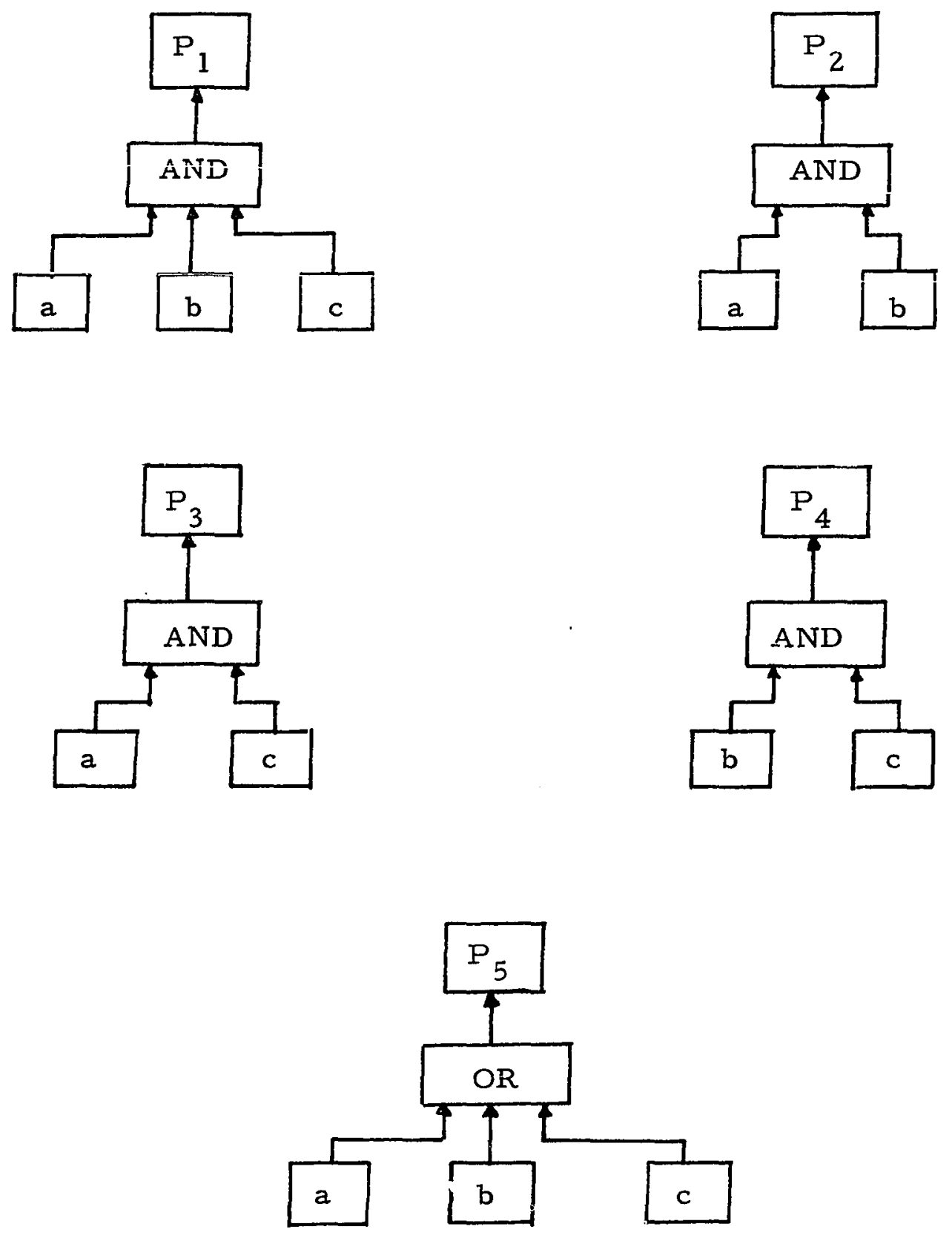

Figure 15. Possible policies for TT feasibility assessment 
The decisionmaking implications of the above five policies are:

$P_{1}: \quad$ This policy statement requires that the required resource must be locally available and be productive and its cost be reasonable, if the $\mathrm{TT}$ is to be labeled feasible. If the required resource satisfies this policy statement, it follows that the feasibility of $\mathrm{TT}$ is established and no constraints exist which can make it infeasible. Ideally, one would like all resource variables to satisfy this policy statement. Unfortunately, constraints always exist in the real world.

$\mathrm{P}_{2}: \quad$ This policy statement requires that the resource must be available domestically, and be productive, but its cost does not have to be reasonable. Naturally, if there are constraints on the cost (as there usually are), the resource variable that meets only this condition should be examined carefully by the decisionmaker to determine means to lower its cost. In this case, we say that the $\mathrm{TT}$ is feasible under constraints (cost in this case).

$\mathrm{P}_{3}$ : Here, the policy statement requires that the resource must be available locally and be of reasonable cost, but its productivity may be low. This is often the case in developing countries. The productivity of the human resources is usually low in a developing country. Thus, under this statement, TT is allowed to be feasible, with the requirement that the decisionmaker pay special attention to the improvement of the productivity of the resource in question.

$\mathrm{P}_{4}: \quad$ Here, it is required that the resource be productive, its cost be reasonable, but it may not be available locally. Again, this is 
the situation often encountered by a developing country. In this case, the resource may be imported but then its cost may not be reasonable. The decisionmaker then may have to make trade-offs.

$\mathrm{P}_{5}: \quad$ This policy statement relaxes requirements on any two of the three measures. Hence, this is considered an unrealistic policy. If a resource variable can satisfy only $\mathrm{P}_{5}$, the $\mathrm{TT}$ under investigation is labeled infeasible.

Thus, these five policies generate a continuum with two extremes-feasible $\left(P_{1}\right)$ and infeasible $\left(P_{5}\right)$. Between the two extremes, there are possibilities that $\mathrm{TT}$ is feasible under constraints of cost $\left(\mathrm{P}_{2}\right)$, productivity $\left(\mathrm{P}_{3}\right)$ or availability $\left(\mathrm{P}_{4}\right)$

The procedure for estimating the feasibility of a TT can now be summarized. The steps of the procedure are:

(i) Use $(T \times R)_{f}$ matrix

(ii) For each resource variable having a value of 1 in the $(T \times R)_{f}$ matrix, develop binary specifications for the availability (a), productivity (b) and opportunity cost (c) of the resource variable.

(iii) Utilizing the binary specifications, develop the policy statements $P_{1}-P_{5}$. Identify all the pairs $\left(R_{i}, P_{k}\right)$ where in $P_{k}=1$. For each $R_{i}$, there will be at least one $P_{k}$ having a value of 1 . There are $\mathbf{i}=1, \ldots, \mathrm{n}$ resource variables and $\mathrm{k}=1, \ldots, 5$ policy statements This procedure yields a policy vector $P=P\left(R_{i}, P_{k}\right)$.

(iv) The vector $\mathrm{P}$ can be used to evaluate the feasibility of TT. 


\section{Specifically:}

(a) if the vector component $P\left(R_{i}, P_{k}\right)=1$ and $P_{k}=P_{1}$ (i.e., for the $R_{i}, P_{1}=1$ ), the $T T$ is feasible

(b) if the vector component $P\left(R_{i}, P_{k}\right)=1$ and $P_{k}=P_{5}$, the $\mathrm{TT}$ is infeasible.

(c) if the vector component $P\left(R_{i}, P_{k}\right)=1$ and $P_{k}=P_{2}$ or $P_{3}$ or $\mathrm{P}_{4}$, the $\mathrm{T} T$ is feasible under constraints.

In case (c), the decisionmaker must carefully evaluate the implication of the corresponding policy statement requirements.

\subsubsection{Efficiency of TT or Applicability of Transfer Mechanisms}

The efficiency of $\mathrm{T} T$ can be expressed in terms of applicability of transfer mechanisms. Ackoff has an interesting way of expressing the notion of efficiency [1]. Using Ackoff's notion, the efficiency of a T T is defined as the likelihood that the desired outputs of TT would occur, given the inputs necessary for transfer. This likelihood in turn depends on how well a transfer mechanism can be applied for a particular TT. The applicability depends on several factors. For our purpose, the important factors are specified by considering:

(i) the number of times the mechanism has been used successfully in the past to transfer the technology in question. For the "firsttime" transfers, this factor is obviously irrelevant. Let us denote this factor by the letter $\mathrm{x}$. 
(ii) the time involved to transfer the technology in question, using the mechanism. Let this factor be denoted as $y$.

(iii) the projected usefulness of the mechanism, for the transfer of technology in question. Let this factor be denoted as $\mathrm{z}$.

Then, the applicability of a particular transfer mechanism can be expressed in various ways, using the factors $x, y, z$. In the rest of this discussion, the terms "applicability of transfer mechanisms" and "efficiency of TT" will be used inter changeably, i.e. they are considered synonymous.

Now, recall that the $(\mathrm{TxM})_{\mathrm{f}}$ matrix identifies transfer mechanisms applicable to the TT being investigated. Hence, for each entry having a value of 1 in the $(T \times M)_{f}$ matrix, we wish to specify a binary number signifying the nature of $x, y$, and $z$. To do this, we generate the following questions:

(a) how many times the mechanism has been used in the past for the TT being investigated? If the mechanism has been used successfully, more than once, $x=1$; otherwise, $x=0$.

(b) is the time to transfer the technology, using this mechanism, reasonable? "Reasonable" can be defined in terms of the timespan of interest to the planner and policymaker concerned with the TT. For instance, five year plans of many developing countries could not allow transfer times greater than their target dates. If the answer to the question is yes, $y=1$; otherwise, $y=0$. 
(c) is the mechanism expected to be viable in future? Information to answer this question exists in literature (e.g., with respect to the mechanism of patents, the literature [17] shows that patents are declining in importance as TT mechanisms.) If the transfer mechanisms is expected to be viable in future, $z=1$; otherwise, zero.

These questions can be answered based on literature on $T \mathrm{~T}$, analysis of transfer mechanisms and judgment of the analyst.

Having developed the values of $x, y$, and $z$, it is possible to generate suitable policy statements to estimate the applicability of a transfer mechanism or the efficiency of a TT. The possible policy statements are:

$Q_{1}:$ the transfer mechanism is applicable iff $x \Lambda y \Lambda z=1$

$Q_{2}:$ the transier mechanism is applicable iff $x \Lambda y=1$

$Q_{3}: \quad$ the transfer mechanism is applicable iff $x \Lambda z=1$

$Q_{4}: \quad$ the transfer mechanism is applicable iff $y \Lambda z=1$

$Q_{5}: \quad$ the transfer mechanism is applicable iff $x \mathrm{VyVz}=1$

The verbal interpretation of a policy statement is $\operatorname{similar}$ to $P_{i}$ mentioned in the previous section. These policy statements can also be depicted graphically in the manner similar to Figure 15.

The implications of the requirements specified in $Q_{1}-Q_{5}$ can be considered, in a manner similar to $\mathrm{P}_{1}-\mathrm{P}_{5}$, as follows.

$Q_{1}$ requires that the mechanism is considered applicable only if it has been used successfully in past at least once, transfer time is 
reasonable and it is projected to be viable in future. Naturally, if a mechanism meets all these requirements, it is considered applicable without any constraints or conditions, and is the most desirable one to employ for the $\mathrm{TT}$ in question.

$Q_{2}$ implies that the mechanism must have been used in the past and transfer time is reasonable, but it may not necessarily be viable in future. If the technology is a "one-shot" type, the mechanism can be considered applicable since its future applicability is of little concern. $Q_{3}$ implies that the mechanism is applicable if it has been used in the past and is applicable in future, but a long transfer time can be tolerated. For a developing region or country, this policy sounds unrealistic, since the time to transfer technology is one of the most important considerations, as discussed in section 1.2 .

$Q_{4}$ requires that the mechanism be vidble in the future and the time for transfer is reasonable, but it may not have been used in past more than once. For a relatively new technology, it seems reasonable to relax the requirement that the mechanism should have been used in past. Hence, $Q_{4}$ is an acceptable policy only if the technology for transfer is new.

$Q_{5}$ relaxes two of the three requirements. Hence, it is considered inapplicable for the TT in question.

The procedure for estimating the applicability of a transfer mechanism can now be summarized: 
(i) Use the $(\mathrm{T} \times M)_{f}$ matrix

(ii) For each mechanism $M$ applicable to the $T T$ in question (indicated by its value of 1 in the $(T \times M)_{f}$ matrix), develop binary specifications for $x, y$, and $z$, as discussed earlier in this section. There are $\mathrm{j}=1, \ldots, \mathrm{m}$ mechanisms

(iii) For each $M_{j}$, develop the policy statements $Q_{1}-Q_{5}$ using the respective values of $x, y, z$, as outlined earlier in this section. Identify the pair $\left(Q_{k}, M_{j}\right)$ so that for the given $M_{j}$, the corresponding $Q_{k}=1$. Recall that $k=1, \ldots, 5$.

(iv) Thus, for each $M_{j}$ there is at least one $Q_{k}$ having a value of 1. This yields a policy vector $Q=Q\left(M_{j}, \Omega_{k}\right)$.

(v) The vector $Q$ can now be used to estimate the applicability. Specifically:

(a) if the vector component $Q\left(M_{j}, Q_{k}\right)=1$ and $Q_{k}=Q_{1}$, the mechanism $M_{j}$ is applicable under any conditions.

(b) if the vector component $Q\left(M_{j}, Q_{k}\right)=1$ and $Q_{k}=Q_{5}$ or $Q_{k}=Q_{3}$, the mechanism is said to be NOT applicable (for a developing nation).

(c) if the vector component $Q\left(M_{j}, Q_{k}\right)=1$ and $Q_{k}=Q_{2}$, the mechanism is applicable only if the TT is of "one-shot" type.

(d) if the vector component $Q\left(M_{j}, Q_{k}\right)=1$ and $Q_{k}=Q_{4}$, the mechanism is applicable only if the technology for transfer is a new technology. 
Again, there exists an applicability continuum between the extremes -applicable and inapplicable under any conditions. In between the two extremes, we have two special cases: when a transfer mechanism is applicable only if the technology for transfer is "one-shot" type, or new.

\subsubsection{Desirability of the Consequence of TT}

It is difficult to derive a generalized technique for assessing desirability of the consequences of $\mathrm{TT}$, since much value judgment is involved. However, an approach based on Alexander's notion of a "misfit" variable can be developed.

It can be recalled that the variable is labeied "misfit" if it produces a stress between form and context. A proper fit between form and context is derived by eliminating the misfit variables, or by specifying a requirement that the set of misfit variables should be a null set. Such a requirement can then be used to specify desirability of $\mathrm{T} T$ in terms of its impact on recipient's sociocultural and economic environment. Hence, one approach is to call the TT desirable only if the corresponding set of misfit impact variables is a null set. Unfortunately, in most practical applications, such a null set does not usually exist.

The matrix $(\mathrm{T} \times \mathrm{C})_{f}$ indicates sociocultural and economic variables impacted by the technology for transfer. Recall that an entry of 1 in the $(\mathrm{TxC})_{\mathrm{f}}$ matrix indicates that $\mathrm{T} \rightarrow \mathrm{C}$. Now, to estimate desirability, we: 
(a) assess if the impact variable corresponding to a 0 entry is a misfit variable, in the sense that the lack of impact produces a misfit, and

(b) assess if the impact variable corresponding to a 1 entry is a misfit variable, in the sense that the existence of impact creates a misfit.

It must be noted carefully that the assessment (a) and (b) is quite differ ent. In the case (a), the policymaker is concerned because the interaction does not exist while in case (b), he id concerned because the interaction exists. It must also be noted that a variable cannot be labeled misfit until the existence or the nonexistence of an interaction is established.

Using the above conceptual scheme, the procedure for assessing desirability of TT can be summarized:

(i) Use $(\mathrm{TxC})_{f}$ matrix

(ii) Partition the $(\mathrm{T} \times \mathrm{C})_{\mathrm{f}}$ matrix into two submatrices, one consisting of the l's in the original matrix, and the other 0's.

(iii) For each entry in the two submatrices, estimate if misfit can occur, as explained above; if it does, specify a 1 for that variable; otherwise, specify 0 . Enter the 1 or 0 in the new matrix.

(iv) Step (iii) yields two matrices, containing binary specifications for misfit variables. Combine these two matrices, using the logic OR operation, to yield a $(\mathrm{TxC})_{\mathrm{m}}$ matrix. Each row of the $(\mathrm{TxC})_{\mathrm{m}}$ matrix 
contains misfit variables corresponding to a particular row (i.e., a technology attribute) of the $(T \times C)_{f}$ matrix.

(v) The technology attribute is desirable if the set of the corresponding misfit variables is a null set. Otherwise, the policymaker must evaluate the implication of each misfit variable having a value of 1 in the $(T \times C)_{m}$ matrix. Specifically, policies must be designed to eliminate the misfit.

The judgment of whether the technology for transfer is desirable in terms of its consequences can be established by examining the desirability of individual attributes of the technology. It may also be possible sometimes to rank the technology attributes in terms of the degree of misfit. In most cases, at least some entries in the $(\mathrm{TxC})_{\mathrm{m}}$ matrix will be 1 , indicating the presence of a misfit. The best that an analyst can do is to indicate the existence of misfit to the policymaker who is responsible to eliminate the misfit. Vickers [100,p.125] suggests one such interpretation of policy:

"The object of policy at every level is to preserve and increase the relations we value and to exclude or reduce the relations we hate".

To illustrate the above scheme, an example is presented. Consider that we have the following $(\mathrm{TxC})_{f}$ matrix:

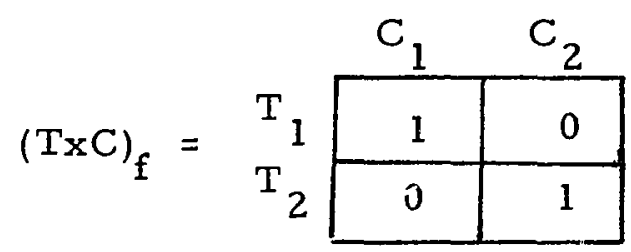


The $(\mathrm{TxC})_{f}$ matrix is partitioned into the following two submatrices:

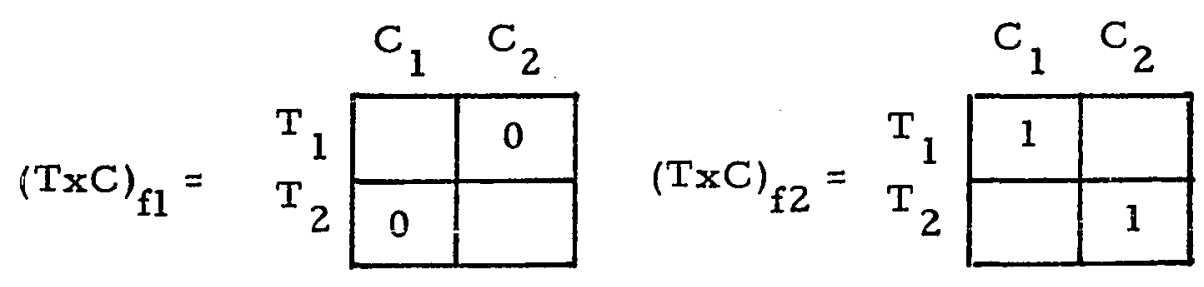

We estimate for each entry whether the existence of impact: $T_{1} \rightarrow C_{1}$, $T_{1} \rightarrow C_{2}$, and the nonexistence of impact: $T_{1} \neq C_{2}, T_{2} \neq C_{1}$, creates a misfit. Suppose that the impact $\mathrm{T}_{1} \rightarrow \mathrm{C}_{1}, \mathrm{~T}_{2} \rightarrow \mathrm{C}_{2}$ does not create misfit and that the nonexistence of impact of $T_{1}$ on $C_{2}$ and $T_{2}$ on $C_{1}$ does. Then, the corresponding submatrices of the misfit variables are:

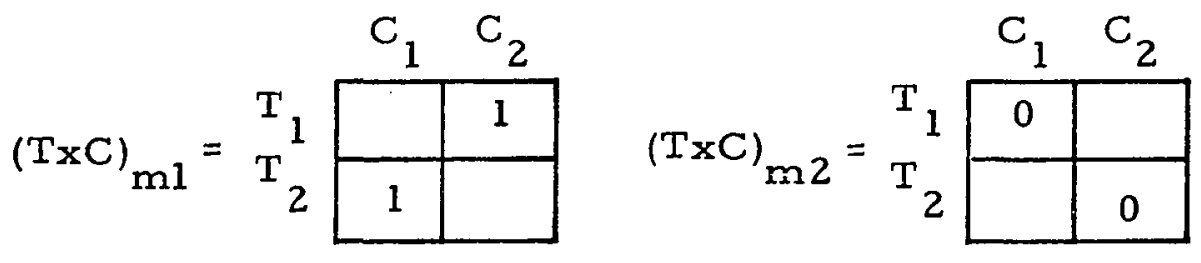

Combining the above two submatrices, we get the $(\mathrm{TxC})_{\mathrm{m}}$ matrix of the misfit variables:

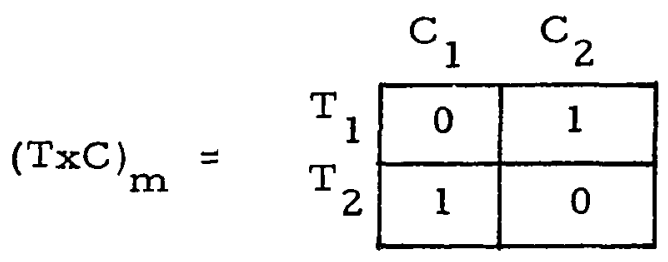

The matrix $(\mathrm{TxC})_{\mathrm{m}}$ indicates that neither the technology att ribute $\mathrm{T}_{1}$ or $\mathrm{T}_{2}$ is desirable because each of them produces a misfit in terms of at least one consequence. The policymaker's task is then to evaluate the the misfits and eliminate them through system improvement and regulation. 


\section{CHAPTER IV}

\section{APPLICATION OF THE PROPOSED IS: TRANSFER OF COMPUTER TECHNOLOGY TO INDIA}

The methodology developed in Chapter III is new for studying TT (Technology Transfer) problems. Hence, it is worthwhile to demonstrate that the method is useful and operational. In this chapter, we describe:

(a) hows and whys behind the selection of technology for transfer donor and recipient countries,

(b) the development of hierarchical structure for the selected $\mathrm{TT}$

(c) an analysis for developing the three performance measures,

(d) implications for policymaking and planning for TT. The overall plan for application is showin in Figure 1.6 . It can be noted from Figure 16 that the development of Levels 1 and 2 an iterative process; seldom is the structure of a complex system developed in one trial. Here, we iterated once around each loop before the structures of Levels 1 and 2 were deemed satisfactory. This process of feeding back information for developing system structure is important. The emphasis 


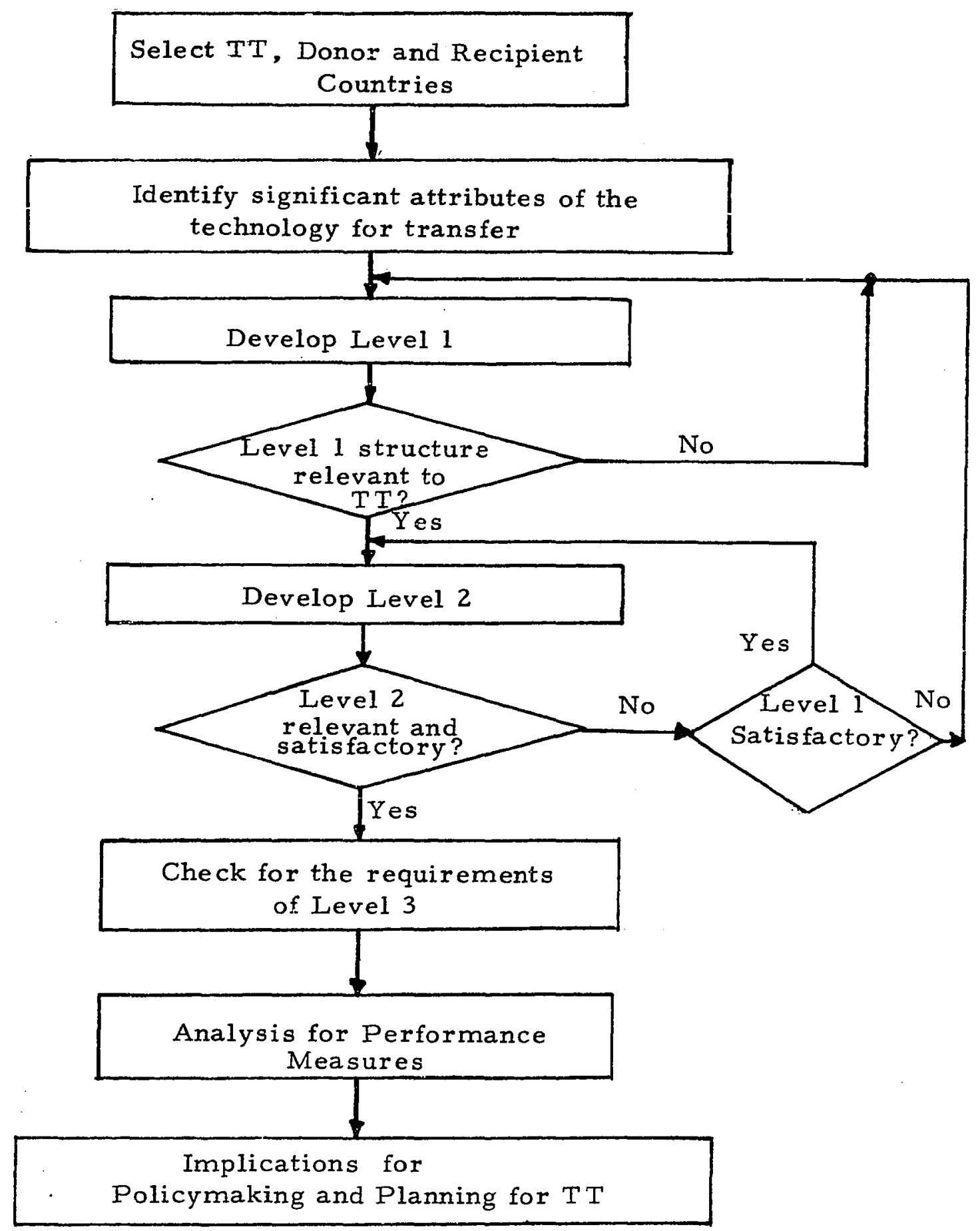

Figure 16: Plan for demonstrating application of the proposed methodology. 
is on illustrating the methodology; the needed techniques such as that for establishing interactions are important but are considered complementary means to reach the ends.

\section{1 Selection of Technology for Transfer, Donor and Recipient Countries}

For our purposes, it was decided to select a $\mathrm{TT}$ whose scope is relatively large, is likely to take place in practical situations, is meaningful and can be purposive. To illustrate the need and usefulness of a muitidimensional approach to $\mathrm{TT}$, we also wished to select a $\mathrm{TT}$ which can significantly impact sociocultural and economic environment of the recipient country. The results of the application should also be useful for practical purposes. Furthermore, it was decided to select a technology which is well established in the donor country but is relatively new to the recipient country. In this respect, horizontal $T T$ is of interest.

For the recipient countries, one of the many developing countries, e.g., India, Peru, Greece, can be selected. For the donor countries, the possible candidates were: U.S.A., Japan and West Germany.

After studying some of the alternative TT candidates, selection of computer technology transfer to India was made. Computer technoiogy ${ }^{3}$ meets most of the requirements cited above. India was selected because: (a) it is a developing country, (b) its potential market for CT

3. Computer Technology will be abbreviated CT in the rest of this dis cussion. 
is formidable, (c) it is felt that CT would be a very useful technology for India, and (d) the writer's personal preference for his native country. The U.S.A. was selected as the donor country because: (a) it is and will continue to be the major manufacturer of CT for some time to come, (b) companies such as IBM are already attempting to enter the Indian market, and (c) it is the leading innovator country in the CT field. It must be pointed out, however, that the main concern here is the recipient country; the donor country is of interest only to the extent that it is willing to export its technology .

\subsection{Application of the Methodology}

Application of the proposed method to CT transfer from U.S. to Irưià involves:

1. development of Level 1 ,

2. development of Level 2,

3. reviewing Level 3 requirements,

4. developing performance measures -- feasibility, applicability and desirability indices.

5. identifying policymaking and planning implications of results of the analysis. As pointed out earlier, two rounds (or one iteration around the loops of Figure 16) were needed to develop Levels 1 and 2 . 


\subsubsection{The Development of Level 1 (Round 1)}

It can be recalled from section 3.7 that Level 1 represents the static structure which specifies variables relevant to the TT in question. These variables are specified for the three critical dimensions -resources, transfer mechanisms and consequences. It is also necessary to list the attributes (components) of the technology for transfer -. $\mathrm{CT}$ in this case. Level 1 is then considered in terms of the relationships of the CT attributes to the three critical dimensions.

\subsubsection{CT Attributes}

A list of CT attributes can be established in various ways; based on the literature on CT, such as reference 67 and $93, C T$ can be characterized in terms of:

(a) Figures of Merit for CT: These can be:

(i) Figures of merit for solid state units, e.g., processing speed,

(ii) Figures of merit for memory units, e.g., storage density,

(iii) Figures of merit for peripherals, e.g., input/ output speed,

(iv) Figures of merit for language.

(b) Subsystems: The major subsystems of a CT can be:

(i) mainframe, i.e., architecture, central processor, memory, 
(ii) peripherals, i.e., external storage, input/ output

(iii) networks, i.e., arrangement of CPU, job entries etc.

(iv) application programming, i.e., software.

(c) Major components: The CT can also be classified into:

(i) hardware, i.e., (i), (ii), (iii) of (b) above.

(ii) software, i.e., (iv) of (b)

(d) Processes: A partial list of representative processes can be:

(i) research and development

(ii) production

(iii) distribution

(iv) programming

(e) Indicators: CT can also be considered in terms economic indicators such as exports attributed to $\mathrm{CT}$, domestic sales, etc.

Thus, CT can be characterized in several ways.

For our purposes, CT attributes should be selected based on their usefulness for TT analysis and design. A CT attribute can be selected by considering:

(i) its ability to identify relevant aspects of CT as related to the three critical dimensions,

(ii) its potential in lending insights into the $\mathrm{TT}$ process, 
(iii) its usefulness for developing interaction matrices,

(iv) its relevance to planning and policymaking related to the transfer of $\mathrm{CT}$,

(v) its usefulness in parametric sensitivity analysis.

Comparison of the four alternatives in terms of these criteria leads to the selection of CT attributes.

Figures of merit provide a useful list of CT attributes, if the purpose is to study capabilities and ultimate potential of CT. Relating figures of merit to resources, mechanisms and consequences can be difficult. They do, however, provide measures useful for developing interaction matrices.

The subsystem classification (b) or (c) represents the relevant aspects of $\mathrm{CT}$ in the three critical dimensions. If one is not careful, further classification of subsystems can become too detailed to be useful. Development of Level 1 in terms of the CT processes and indicators (d and e) does not seem useful for our purposes. First, for the users of this study -- policymakers and planners -- such classification seems too involved. Second, it is necessary to classify the processes into their elements before Levels 1 and 2 structure can be developed. Third, it is doubtful that indicators such as exports attributed to $C T$ transfer can be developed readily for India at the present time.

It seems most usefui then to consider CT in terms of the following attributes: hardware, software and peripherals. This classification is a compromise between the classifications (b) and (c). It is broad 
enough for policymaking and planning purposes and is distinct and specific enough to investigate the three CT attributes separately. When the interactions of these attributes with resource variables, transfer mechanisms and consequences are considered, one can always investigate each CT attribute in detail, if necessary. Reference 67 (pp.21-24) also cites these three subsystems as three important components of the computer industry.

\section{2 .1 .2 Specification of Level 1 Structure}

As discussed in Chapter III, the static structure of $\mathrm{T} T$ is developed by selecting significant variables corresponding to the three critical dimensions of resources, transfer mechanisms and consequences (or impact). The generalized morphological tree of Figure 10 is the primary source for developing a morphological tree for CT transfer to India.

Two criteria are added to the list of variable selection criteria, discussed in section 3.5.1. These are:

(a) the variable must be applicable to CT and India,

(b) the suitability of the variable in light of available data for India in reference sources such as 2, 34, 80, 91 and 97.

These criteria help to "filter" the morphological tree of Figure 10 into the one for CT transfer to India shown in Figure 17.

The selection of variables shown in Figure 17 was based on the morphological tree of Figure $1 \mathrm{C}$ and an extensive literature search 


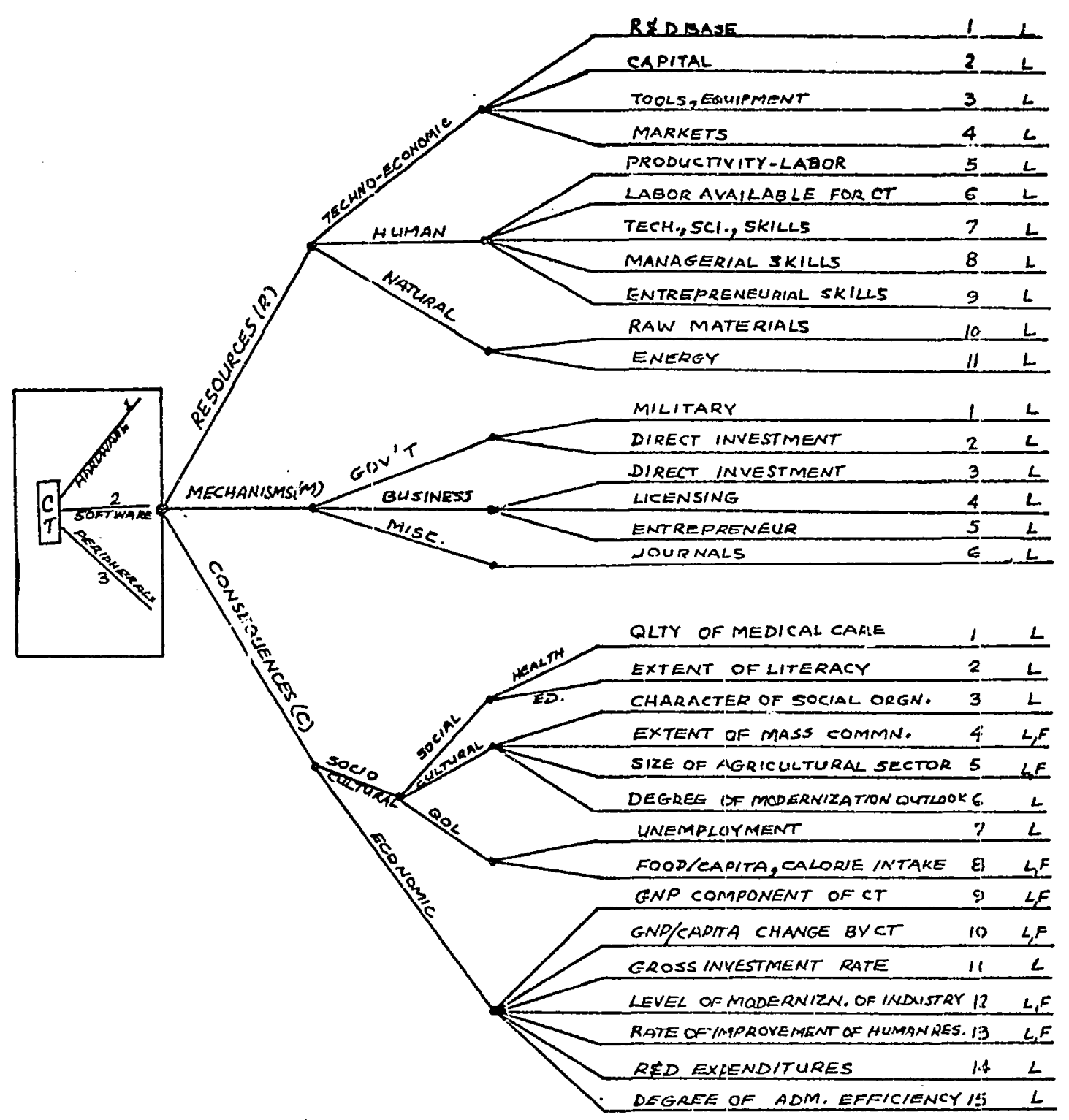

Figure 17. Morphological tree for CT, Round 1 
involving economic, social, cultural, political and computer related studies on India. In particular, references $2,5,15,34,65,67,68$, $71,79,91,93$ and 97 were most useful. The variables selected on the basis of this literature search are indicated by the letter $\mathrm{L}$ in Figure 17. The measures for these variables are those indicated in Figure 10.

In some cases, a variable was found useful on the basis of forecasts that indicated its relevancy to $\mathrm{CT}$ in the future time. This is indicated by the letter $F$ in Figure 17 . It can be observed that none of the variables were selected on the basis of forecasts alone (which indicates the unavailability of good forecasts). However, in some instances, the forecasts strengthen the justification for including the variable.

The justification for including each variable is further discussed in some detail in Table II.

The usefulness of Level 1 , as a general checklist for CT, can now be discussed. The morphological tree conveys explicitly, to the planner and policymaker, the nature of the resources needed for CT, mechanisms available to transfer $\mathrm{CT}$ to India and the sociocultural and economic variables most likely to be impacted by such a transfer. A resource planner can review this specification and then may like to investigate further resource availability, costs etc., before developing Level 2. More importantly, Level 1 information is useful because: (a) the morphological tree indicates to the decisionmaker possible channels open to him (e.g., transfer mechanisms), the requirements of CT transfer (e.g., resources), and environmental changes resulting from 


\section{TABLE II}

JUSTIFICATION FOR SELECTING VARIABLES IN

LEVEL 1, ROUND 1

\section{Dimension: Resource Requirements}

\section{A. Techno-economic Resources}

(1) R\&D Base: Computer industry is highly research

intensive. There is a correlation between research intensity and volume of foreign investment. Japan, for instance, spends more money on research in computer industry than any other Japanese industry. Much of this is on software research [67,pp.56-58].

(2) Capital: The computer industry requires high capital expenditures [67,pp.117-123]. If a country decides to get into the computer business, two types of costs are involved: (a) entry costs, (b) costs for growth. Highest costs occur if a country decides to manufacture locally all the three CT attributes. Alternatives are to import some and manufacture the others.

(3) Tools, Equipments: It is rather obvious that if CT transfer includes manufacturing hardware and peripherals, these are required. For software, they may not be critical requirements. (4) Markets: Size and growth of market for CT is an important resource requirement. The size can be measured in terms of total number of yearly installations. The Indian market for CT, although presently a small one, is potentially large [18, p.122]. The European market, for instance, was quite small in 1963 , about $20 \%$ of 
the U.S. However by 1967 it became quite attractive to the U.S. computer manufacturers as evidenced by their investment in Europe [67, Table 16].

\section{B. Human Resources}

(1) Labor productivity: Productivity of the labor force is an important variable to consicier, since iñ the past, it was a major barrier to successful transfers [18,Chapter 26]. The Indian government and its National Productivity Council is establishing programs to improve labor productivity. It must be fointed out that the productivity of scientific labor is sometimes difficult to assess.

$$
\text { Size of Labor-force Available for CT: Relevance }
$$

of this variable is rather obvious. Statistical data are usually collected in terms of percentage of labor force involved in $R \& D$ and scientific work.

$$
\text { Technical and Scientific Skills: Skills for per- }
$$

forming technical and scientific tasks is an important aspect of the human resource. For instance, Japan who spends substantial sums oí money on software $R \& D$ has found this type of $R \& D$ to be highly labor intensive, requiring less capital inputs than hardware $R \& D$ which also requires scientific skills $[67, \mathrm{pp} .56-58]$.

(4) Managerial Skills: It appears from the literature on CT that most industries that are successful, especially in CT, are usually better managed $[\mathrm{e} . \mathrm{g} .$, see $67, \mathrm{pp} .96-108]$. The management 
aspects of CT can be considered in terms of either input factors -- e.g., internal organizations, tactics utilized by the management, qualifications of the managers, etc., or output factors -- e.g., growth rate, market size, etc. For the present study, our concern is the availability of management skills and its cost, as related to CT and India. Entrepreneuriai Skiils: Roberts [75] and others [e.g., 17] have stressed the relationship of entrepreneurship to TT. For CT, it seems that entrepreneur skills are needed, especially for software, e.g., selling application programming.

C. Natural Resources

(1) Raw Materials: These are required to manu facture hardware and peripherals.

(2) Energy: Electrical energy seems to be the only requirement here.

II. Dimension: Mechanisms A. Government

(1) Military: Previous studies [86,93 pp.140-142] indicate military can be a us eful channel to transfer CT. The growth of minicomputers and special purpose computers is mainly due to the U.S. military. The military is both the financier and customer of CT.

(2) Directi investment: Even in the U.S., direct investment in terms of $R \& D$ expenditures on $C T$ is evident. The 
American computer industry has benefited from government support $[67, \mathrm{p} .129]$. Although in many instances the government has acted as a direct supporter of CT development, no explicit government policies existed in relation to computer industry until $1963[67, \mathrm{p}, 129]$. For developing countries, such as India, investment in the public sector is widespread. Banks with deposits of more than 1 crore rupees (approximately 1.5 million U.S. dollars) have been nationalized, increasing the capacity of the central government for direct investment. Bharat Electronics, a public sector investment, has started licensing agreement with ICL (International Computer Limited), U.K. [18,p.120]. Thus, direct investment by the Indian government is a channel that must be considered here.

B. Business

Direct Investment: The computer industry is highly international. Companies such as IBM and GE have large scale, multinational facilities. The investment can occur in various forms: e.g., manufacturing outside the country with heavy investment in software. Thus, market share can be high even if the production is low. For instance, IBM's facilities in Switzerland and Austria [67,pp.78-80].

The direct investment in foreign countries is entirely dominated by U.S. companies. The growth of these companies have prompted them to invest abroad and take over financially troubled companies (e.g., Olivetti). The channel to transfer CT via direct investment has proved viable $[67, \mathrm{pp} .78-80]$. 
Licensing: Reference 67, Table 6 lists a number of companies in Japan and Europe who have developed CT under licensing mainly from the U.S. companies.

There are two types of licenses: (a) patent licenses, and (b) manufacturing licenses. In the computer industry, basic inventions and important developments are protected by patents $[67, \mathrm{p} .44]$. However, most $\mathrm{CT}$ transfers occur utilizing manufacturing licensing. Fees for manufacturing licenses are much higher than patent licenses $[67, \mathrm{pp} .44-49]$.

Although American companies have dominated in supplying licensing agreements, recently Japanese and U.K. companies have been producing general purpose computers without American licensing (e g., Fujitsu and ICL). Countries like India may seek licens ing agreements with these companies, if costs are reasonable. In any case, licensing seems to be an appropriate channel to consider here. (3) Entrepreneur: Entrepreneur seems to be a useful mechanism to transfer CT, especially software [e.g., 67,p.22]. Due to the scope of CT transfer anticipated for India, a single entrepreneur may not be a viable channel. However, for special purpose minicompleters and application software, the entrepreneur has been found a useful transfer mechanism (e.g., small data-processing outfits in the U.S.). Thus, it seems necessary to include this channel for the present analysis. 


\section{Miscellaneous}

Journals: On a smaller scale, journals seem to be applicable in CT transfer, especially for software. Due to the increasing number of publications on $C T$ and related subjects, it seems useful to include this mechanism also.

III. Dimension: Consequences

A. Socio-cultural

(1) Quality of Medical Care: Computers are finding increasing use in health care, e.g., as a diagnostic tool [93,p.94]. For countries like India, the impact of CT on medical care can be enormous. Life expectancy in India is approximately 40 years [80,p.198]. Many die because of inadequate medical care. A proper use of CT can help in extending medical care to the needy especially since the supply of doctors is not very high.

(2) Extent of Literacy: Computers can extend the a vailability of education to larger masses of people through instructions via TV and computer-aided instructions $[91, \mathrm{pp} .203-216 ; 18, \mathrm{p} .118]$. India already has made agreements with NASA to lease channels on their Applications Technology Satellite to beam educational programs to rural India.

(3) Character of Basic Social Organizations: The information revolution accelerated by CT seems to have affected the social fabric of U.S., e.g., credit card society [91,pp.93-103]. In 
India, the basic social organization is the joint family system. The evolution of CT will tend to drift people from old ideas and traditions. For instance, installment buying, once considered undesirable and unprestigeous, has already picked up in big cities like Bombay and New Delhi. The impact of CT on the basic social organization of India is inevitable when and if $\mathrm{CT}$ is transferred to India in large doses.

(4) Extent of Mass Communication: Computers have significantly changed the communication links in the Western World, especially in the U.S. CT can make information available in 'instant' form [91,pp.130-144]. India, suffering from a serious lack of widespread communication links, can benefit from CT applications. For instance, India has 1.6 telephones/1,000 population compared to U.S.'s $481.4[92$, Table 4.7].

(5) Size of Agricultural Sector: India being predominantly an agrarian society, it seems relevant to include this variable in Level 1 .

(6) Degree of Modernization Outlook: It seem.s important to consider this variable in Level 1. References 2, 18, 77 and 78, support the view that modern technology, such as CT, can affect the modernization outlook of a developing country [e.g., 2, Chaper VIII; i 8, Part V).

(7) Unemployment: Widespread unemployment can have serious consequences on the social character of a country. Also, 
it seems that the social and cultural impact of unemployment is less obvious and little attention is paid to it [e.g. see 18, Chapter 26].

(8) Food/capita, Calorie Intake: The calorie intake per capita per day of an Indian is 2,110 compared to the U.S.'s 3,140 [92, Table 4.11]. CT, through its impact on agriculture, can affect this variabie. Therefore, it seeñs necessary to include this variable in Level 1.

(9) The following economic variables were selected for Level 1: GNP (component of CT), GNP/ capita, Gross Investment Rate, Level of Modernization of Industries, Rate of Improvement of Human Resources, R\&D expenditures and Degree of Administrative Efficiency. All of these variables are relevant to an analysis for CT transfer to India. Relevancy was established by consulting studies, forecasts and discussions in many references. The references, found most useful, are those mentioned on p.102. The relevancy of these variables in most cases is obvious. 
CT transfer (e.g., consequences), (b) the assumptions underlying Levels 1 and 2 development are explicit, and (c) it provides flexibility by allowing the possibility of adding or deleting branches or variables from the tree. Also, some of the information provided by the morphological tree may not have been obvious a priori to a decisionmaker. For instance, the resource requirement of availability of management and entrepreneurial skills is often overlooked by Indian planners and policymakers.

No attempts were made to transform the morphological tree into a relevance or a decision tree. The possibility that this can be done is obvious from Figure 17.

Two of the outputs of Level 1 - relevant variables and their associated measures - - are useful in developing interactions for Level 2 . It can be noted that the development of Level 2 , without the outputs of Level 1, would be quite difficult indeed.

\subsubsection{Development of Level 2 (Round 1)}

At Level 2, interaction between variables specified in Level 1 are developed. The synthesis of interaction matrices yields the TT structure. As discussed in section 3.8, interaction can be specified as: binary, on a continuous $0-1$ scale, or in a functional form. Presently, it is not possible to employ the last approach since meaningful functional relationships do not exist. The choice then is between a binary or a continuous scale. 
The following factors ?ed to the selection of binary specifications: (a) efforts required to specify interaction on a $0-1$ scale are substantial and appear quite ambitious for the present study; (b) it seems advisable, as the first step at least, to consider system behavior at the extreme values of a variable, i.e., 0 to 1 . If the behavior is interesting at the two extremes, there is a good reason to interpolate on a 0-1 scale during a second stage of analysis; (c) it appears (and as will be demonstrated later) that the results obtained using binary interaction can lend useful insights into planning and policymaking related to $\mathrm{TT}$. If the statement (c) is true, the results provide sufficient proof of the usefulness of the proposed methodology; it is sufficient in the sense that the method answers the important questions concerning feasibility, efficiency and desirability of TT. It should also be noted that the proposed methodology remains unchanged whether interaction is specified in a binary, continuous or functional form.

We need to develop the following seven interaction mairices: (TxR), (TxM), (TxC), ( $\mathrm{RxR}),(\mathrm{MxM}),(\mathrm{CxC})$, and $(\mathrm{R} \times \mathrm{C})$. The interactions will be interpreted in the manner described in Table I. In section 3.8.1, various ways to establish interaction were specified. Here the specification that an interaction exists (1), or does not exist $(0)$, is established by: (a) using the results of previous statistical studies [e.g. 2], (b) plotting the available data to estimate the relationships involved [e.g., 92], (c) using the data on correlation between variables, 
established in studies on developing countries $[2,23]$, and (d) relevant, available forecasts related to the computer industry $[67,93]$.

To better appreciate the explanations for interaction specifications, a short discussion on computer technology and its implications for India is provided.

The computer industry is the key element in the information revolution. Its importance lies not only in its economic output, which is considerable, but its far-reaching effects on the whole economic, industrial and social structure of a country. In 1950, the computer industry did not exist p:operly; by 1966, the production of digital computer systems in the Western World was around $\$ 4$ billion. In 1966 , the U.S. production amounted to $\$ 3.2$ billion or $0.46 \%$ of the U.S. GNP [ó7,pp.15-17]. The number of computers installed in the Western world is shown in Figure $18[67, \mathrm{p} .16]$. On the basis of 12 operators per computer system, one can estimate that the number of people employed in the utilization of computers in 1966 were 350,000 in the U.S. alone. Based on the available data, U.S. accounts for approximately $95 \%$ of the Western World's production of computers as shown in Figure 19.

India had 50 major computer installations in $1969[18, \mathrm{p} .120]$. The Indian government itself has started importing computers, organized training facilities and assembling complexes are coming up. The leading electronics firm in India, Bharat Electronics, has signed an 


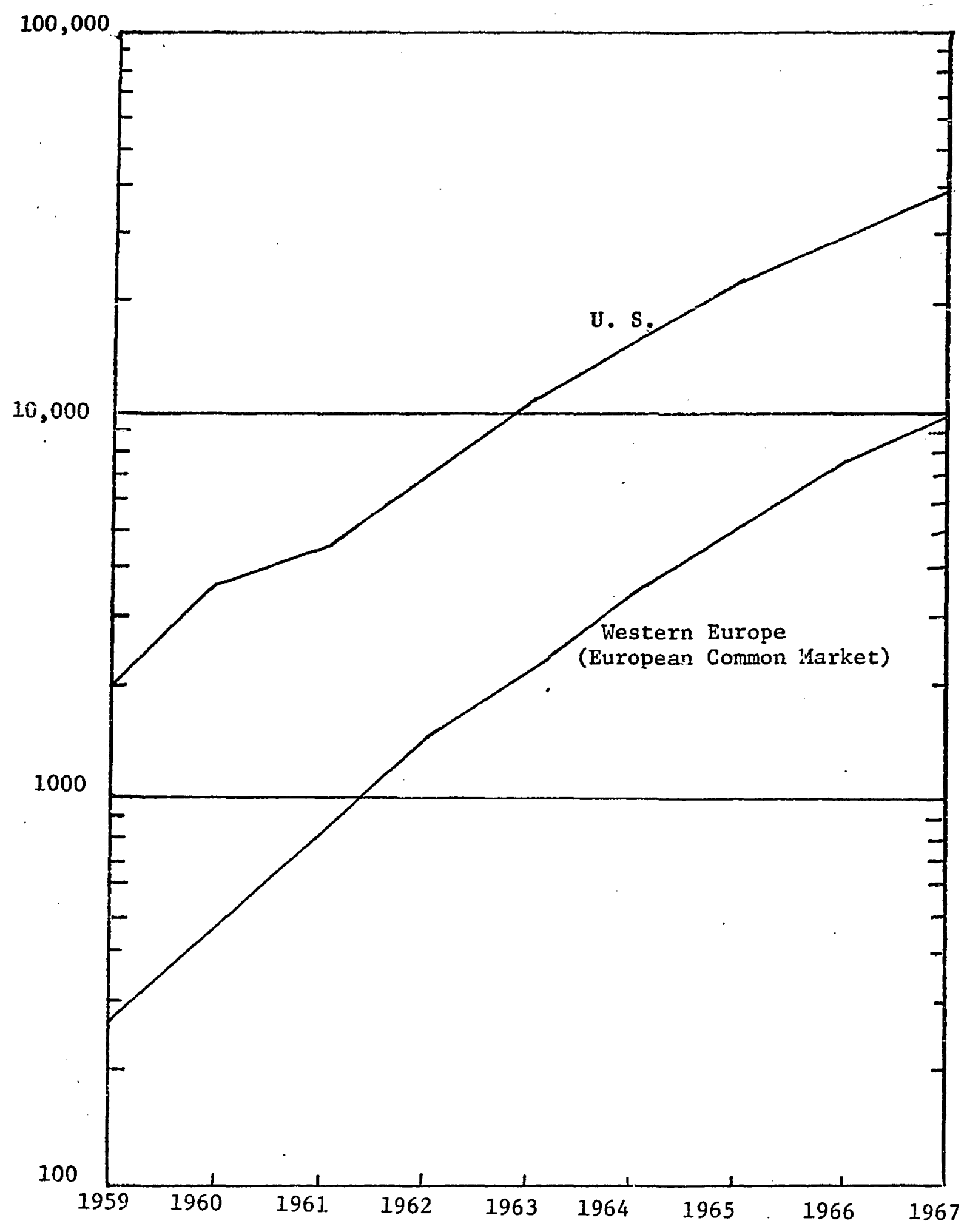

Figure 18. Number of computers installed [67,p.16]. 


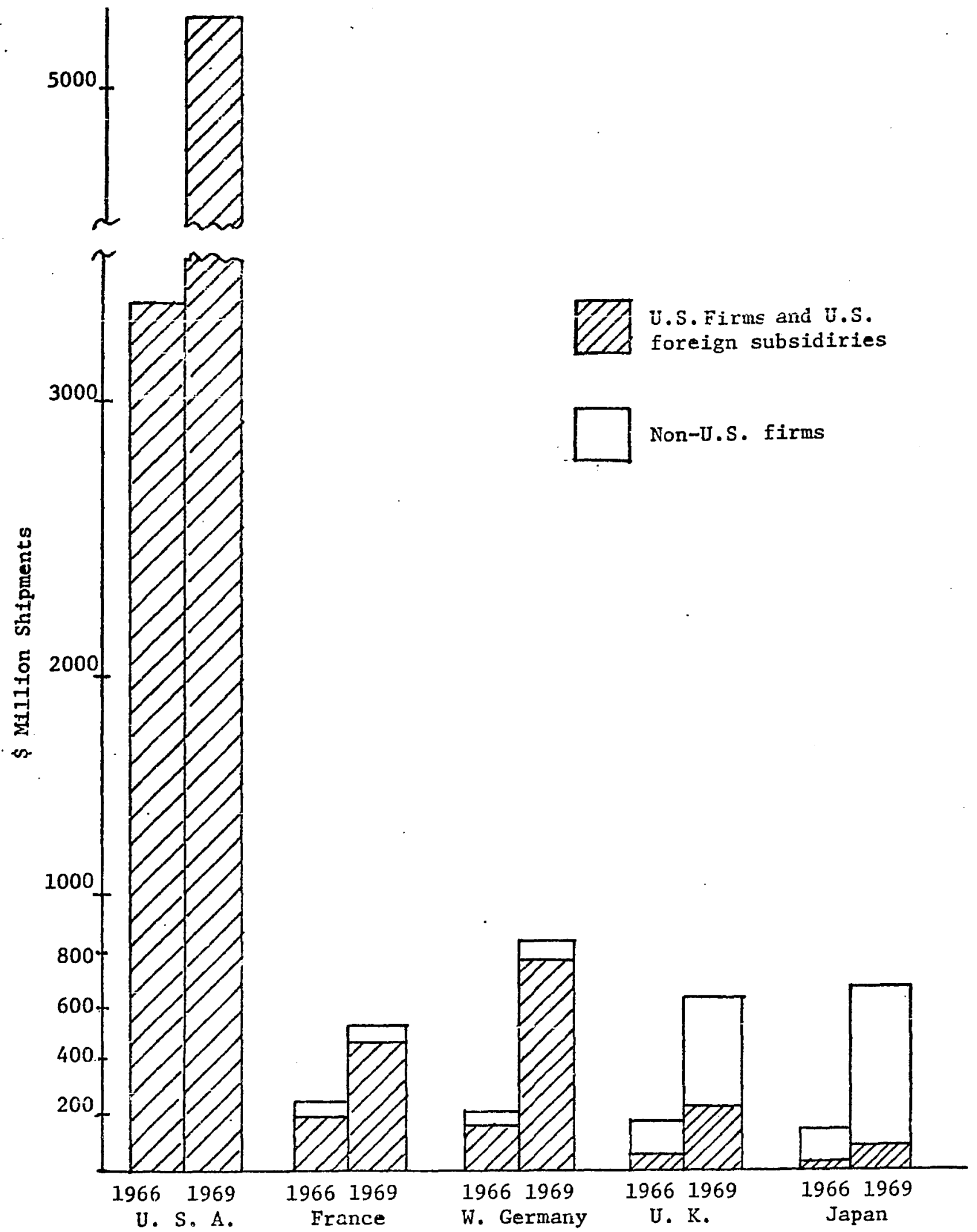

Figure 19. The world computer industry in 1966 and 1968 [67,p.19] 
agreement to assemble ICL (Internationai Computers Limited, a UK firm) computers. Butani states $[18, p .116]$ :

"Institution quick on uptake are organizing seminars and courses, for private corporations, with computer systems, have to find programmers and other high sounding personnel, if the computers are to be anything more than the prestige items. Whole computer departments are coming up providing employment to our mathematical boys, who never had more than an abstract chance in their lives".

Thus, the computer revolution has started slowly in India. Two different representations of computer growth are provided in Figures 20 and 21.

Based on the discussions throughout references 67 and 93 , it seems that the important resources needed for the computer industry are human and techno-economic. Natural resources, mainly the raw materials (semiconductors, magnetics, etc.) and electrical energy, are not critical as far as India is concerned, since these requirements are modest indeed. The major human resources of interest are: productivity, available labor force for CT, technical, entrepreneurial and managerial skills. R\&D base, financial, tools, equipments, and markets are the major techno-economic resources needed. The Indian market for computers is quite large [18, Chapter 17]. The field of education alone is estimated to be the largest single market for computers $[18, \mathrm{p} .118]$. It seems that the emphasis will be on the utilization of the computer, rather than on its manufacturing. Reference 67 , the OECD report, states: 


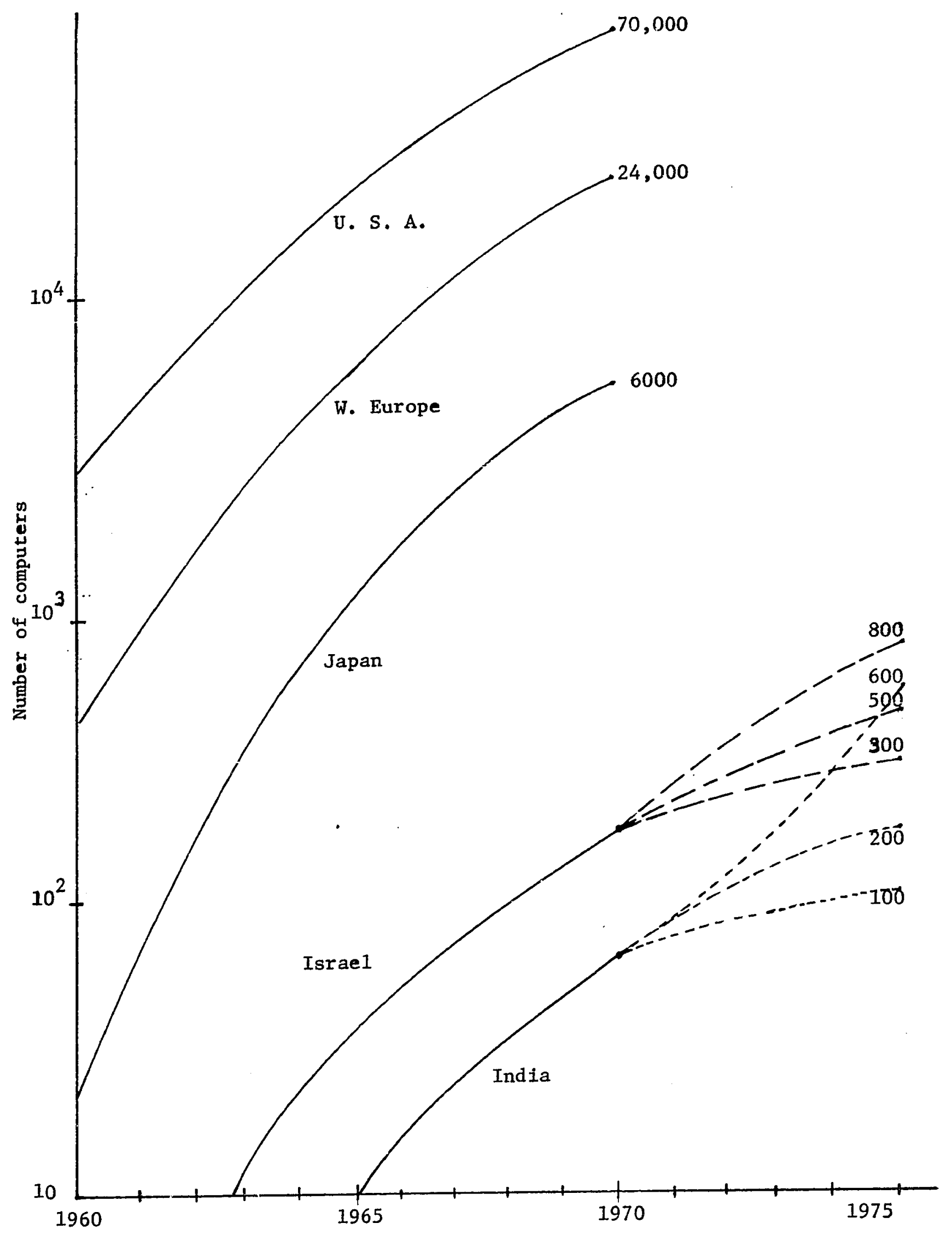

Figure 20. Growth of computers in several countries [93,p.10, except India]. 


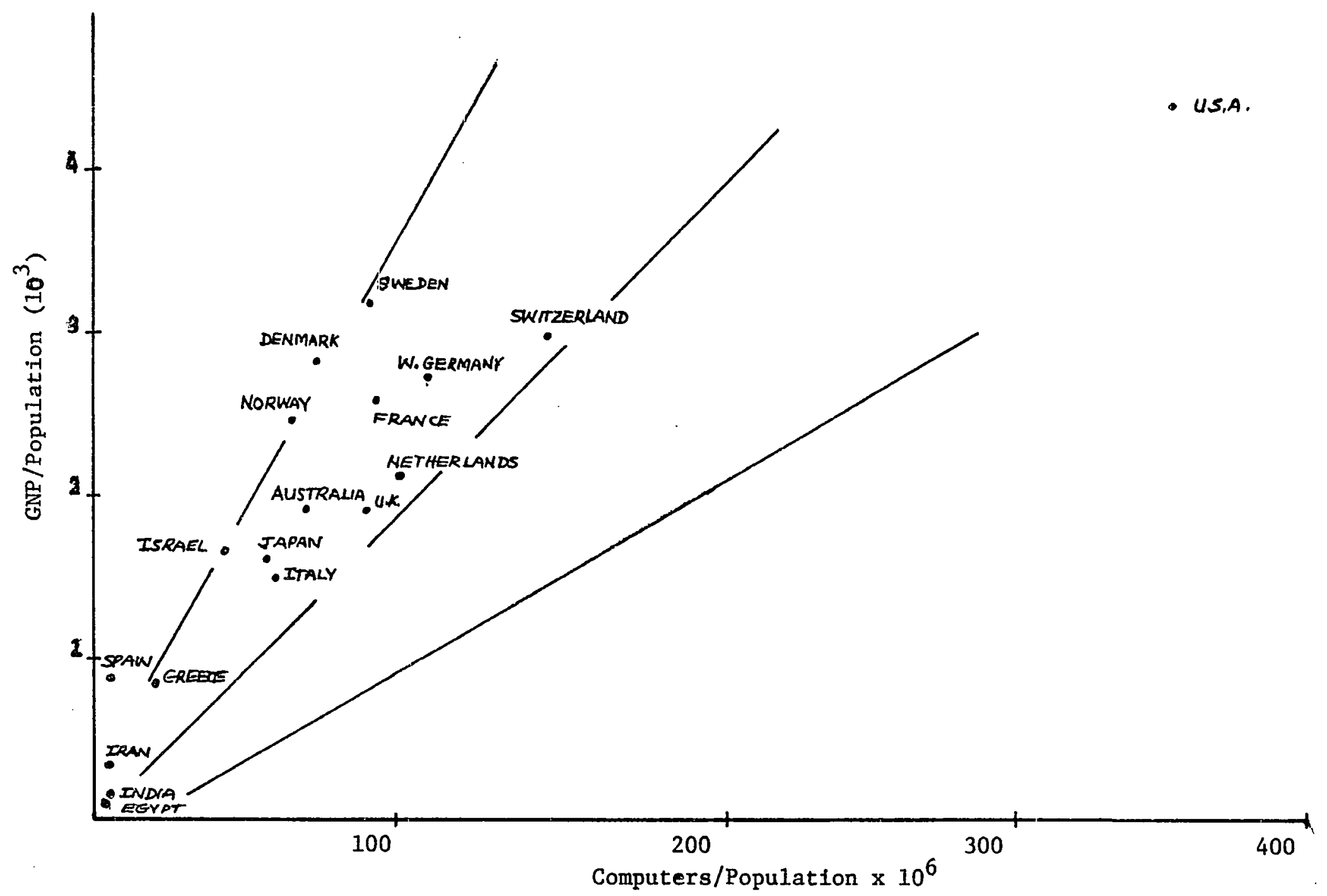

Figure 21. GNP and number of computers [93,p.27, except India]. 
"Up to now, manufacturing computers has been one of the key issues; but in the future, the most important aspect of computer technology could well be the utilization of the tool, rather than the manufacturing" [p. 148].

The computer is expected to diffuse knowledge the way the printing presses did in early days. The evolution of computer usage is shown in Figure 22. It is suggested that India could follow this evolution of computer usage, considering that it is at the stage where France was in 1958. (For instance, see the discussion in 18, Chapter 17).

The mechanisms employed in the past for CT transfer have been licensing and direct investment by business, supported by enterpreneurial efforts, mainly in the software area. In India, most heavy investments are made in the public sector. Hence, direct investment by the government is a likely transfer mechanism. Business is often allowed a joint venture with the government. The militaxy channel, although not effective in the past, may gain importance in the future because of India's entry into the manufacturing of advanced aircraft navigation and guidance systems and the establishment of a modest military aerospace program. The journals can also be useful in the transfer of software ideas.

The consequences of CT on India's socio-cultural environment are more difficult to assess. However, its impact -- direct or indirect -- on various socio-cultural variables will likely take place. The impact of computer utilization in education and medical care could be significant. Reference 91 traces the impact of computers in U.S.A. 


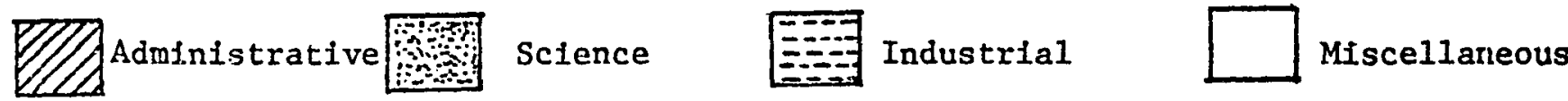
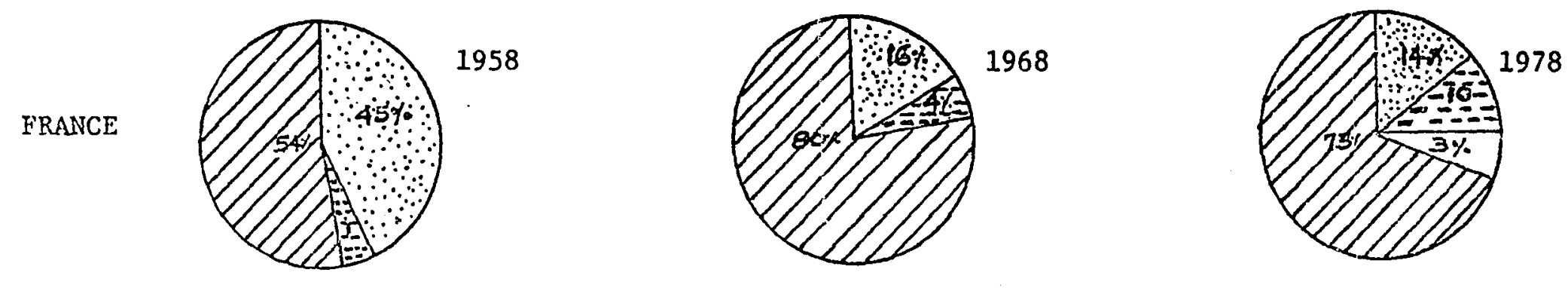

U. S. A.
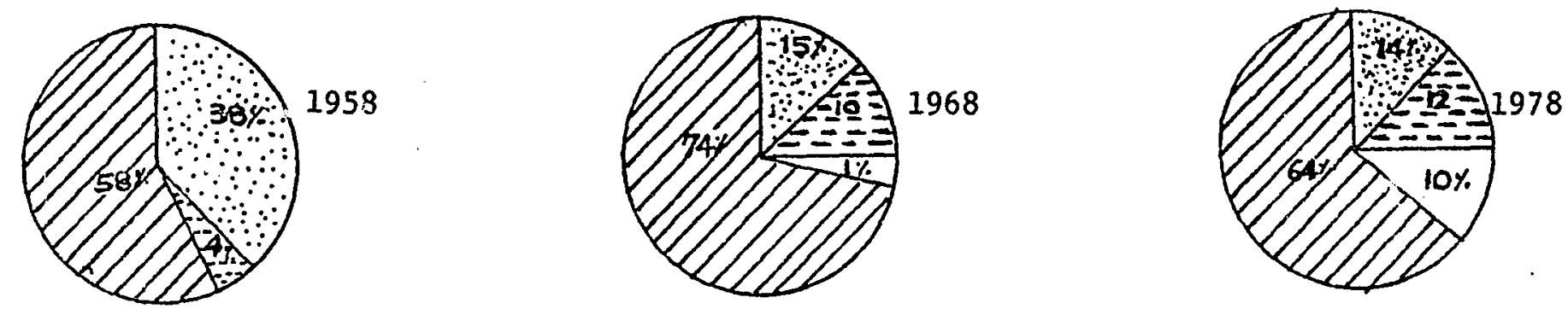

Figure 22. Development of computer usage: France versus U.S.A. [93,p.18]. 
on a wide range of variables such as social, cultural and political. Considering that India is presently where the U.S. was in the late fifties, the impact of $\mathrm{CT}$ can be massive. The economic consequences of $\mathrm{CT}$ transfer are easier to assess since enough precedences and analogies exist. An interesting way to depict socioeconomic status of various nations is provided in Figure 23.

The above discussion leads to the development of interaction matrices for round 1. Figure 24 (a) represents interactions between the three technology attributes and the resource variables. This is the (TxR) matrix. Figures 24 (b), (c) represent (TxM) and (TxC) matrices. Figure 24 (d), (e), (f) represent the self interaction matrices: (RxR), $(\mathrm{MxM})$ and $(\mathrm{CxC})$. Figure $24(\mathrm{~g})$ represent the cross-interaction matrix $(\mathrm{RxC})$.

The interactions a re explained in Table III. The elements of column 1 correspond to the elements of the respective matrices of Figure 24(a)-(g). For instance, the element $(1,1)$ under the (TxR) heading refers to the interactions between $T_{1}$-hardware, and $R_{1}$ $R \& D$ base.

Using the technique for synthesizing interaction matrices, outlined in section 3.8 .3 , the seven matrices of Figure 24 are synthesized to yield the $(T x R)_{f},(T x M)_{f}$, and $(T x C)_{f}$ matrices for round 1 . These matrices take into account direct and indirect interactions between the technology attributes and resource variables, mechanisms and consequences. The synthesized matrices are shown in Figures 25, 26, and 27. 


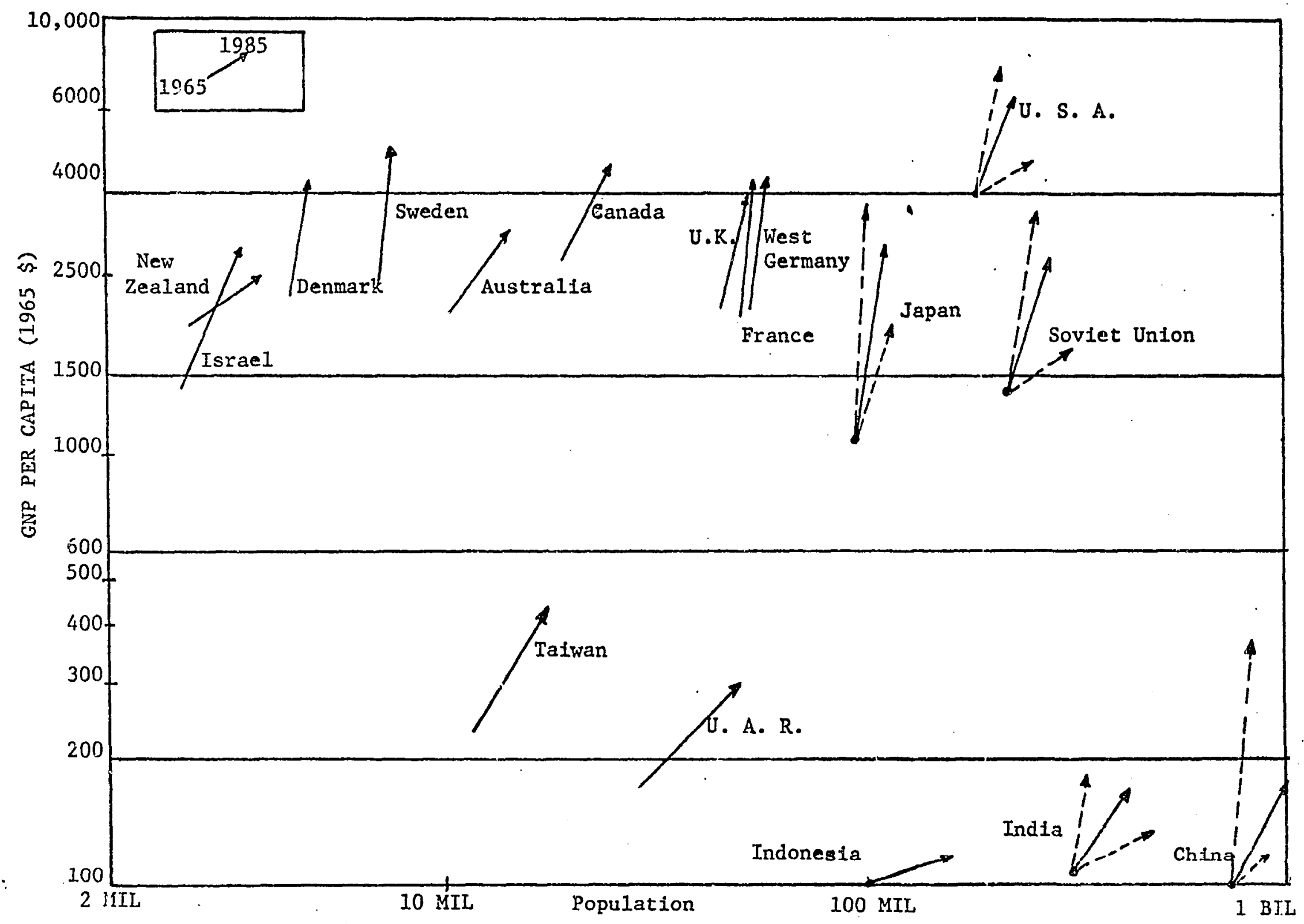

Figure 23. Socioeconomic status of nations $[93, p .29$, except India]. 


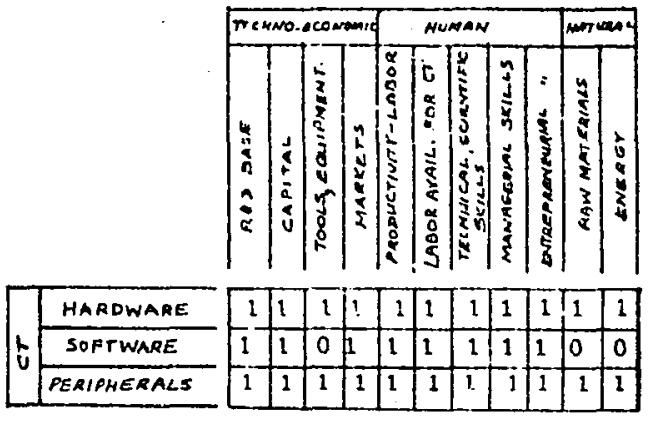

(A) $(2 \times a)$

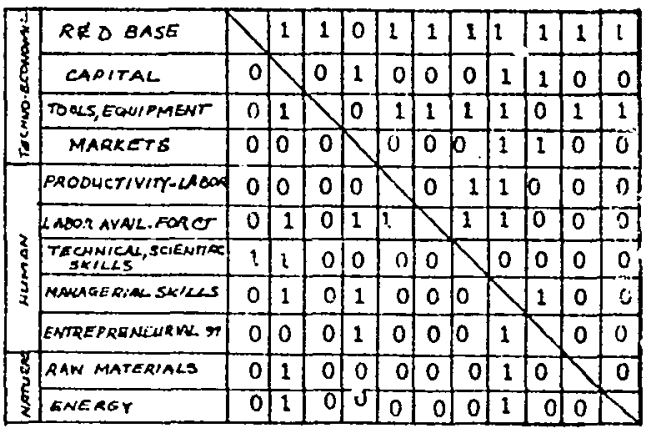

(1) $(\mathrm{AxB})$

Figure 24. Interaction Matrices, Round 1

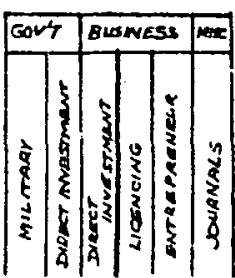

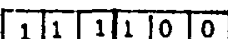

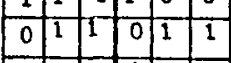

\begin{tabular}{|l|l|l|l|l|l|}
\hline 1 & 1 & 1 & 1 & 0 & 0 \\
\hline
\end{tabular}

(b) $(\mathrm{I} \times \mathrm{h})$

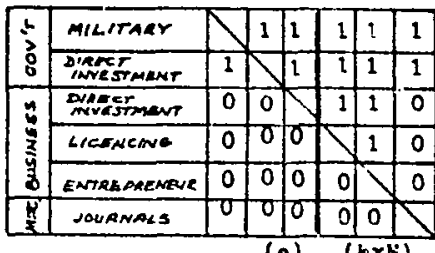

(e) $\frac{(n \times k)}{(n)}$

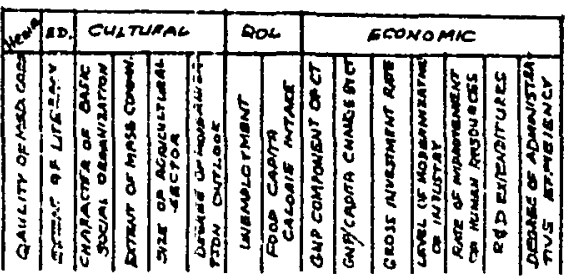

\begin{tabular}{llllllllllllllll}
0 & 0 & 0 & 0 & 0 & 0 & 0 & 0 & 1 & 1 & 0 & 1 & 0 & 1 & 1 \\
\hline
\end{tabular}

\begin{tabular}{llllllllllllllll}
1 & 0 & 0 & 1 & 0 & 0 & 1 & 1 & 1 & 0 & 1 & 0 & 1 & 1 \\
\hline
\end{tabular} \begin{tabular}{llllllllllllllll}
\hline 0 & 0 & 0 & 0 & 0 & 0 & 0 & 1 & 1 & 0 & 1 & 0 & 1 & 1 \\
\hline
\end{tabular}

\section{(\&) $(\Gamma \times C)$}

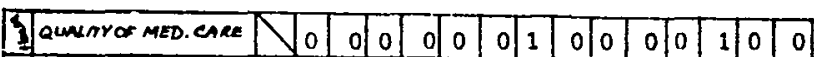

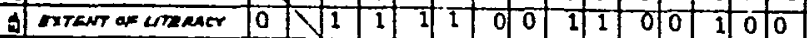

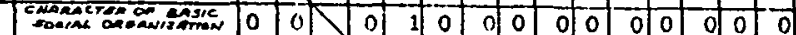

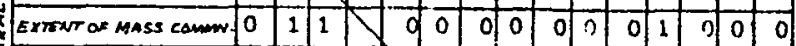

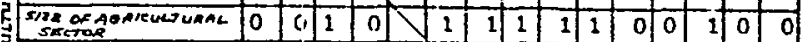

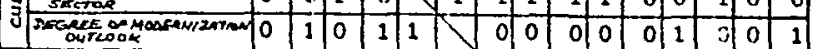

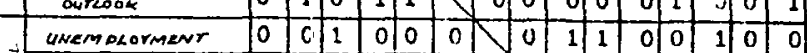

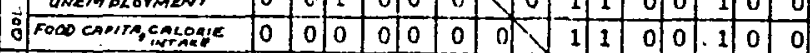

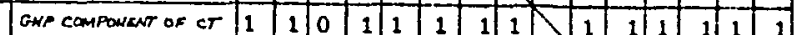

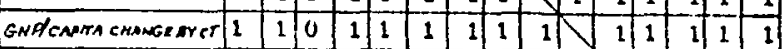

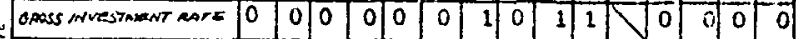

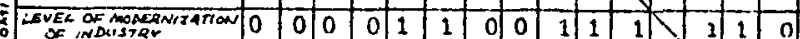

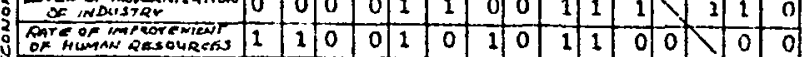
end expenarrues 0000000

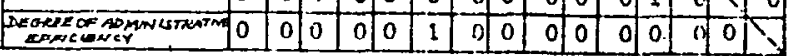
(r) $(\mathrm{ExC})$

\begin{tabular}{|c|c|c|c|c|c|c|c|c|}
\hline SASE & 0 & D] $\frac{n}{n}$ & 00 & \begin{tabular}{l|l}
0 & 0
\end{tabular} & 010 & 0 & & \\
\hline CAPITAK & 0 & 010 & 00 & $0 \longdiv { 1 }$ & $0 \longdiv { 1 }$ & $\mathrm{l}$ & 0 & \\
\hline 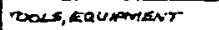 & & $0 / 0$ & 00 & $0 \%$ & तT & 010 & $\begin{array}{lll}17 & \end{array}$ & \\
\hline $\operatorname{mask}=T_{S}$ & 0 & 00 & 00 & $0] 1$ & $0 \mid 0$ & 01 & to & \\
\hline 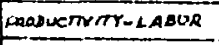 & 0 & 00 & 0 & 0 & 00 & 1 & 1 & \\
\hline LABOR AYAR FOC CT & 0 & 00 & 00 & 0 - 1 & $0 \%$ & 00 & 11 & \\
\hline 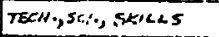 & 0 & 00 & 00 & 10 & की & oto 0 & 10 & \\
\hline MANAGER/PL SKRLS & 0 & 00 & 00 & 10 & oी & 0 & 11 & \\
\hline ENTERPRENARRAK "I & 0 & 0.0 & do & $0 \longdiv { 0 }$ & 0 & 0 & 0 & \\
\hline RAW' MUTERAALS & 0 & do & 00 & $0] 0$ & to & 6) & $0 \div 01$ & \\
\hline ENERER & n & do & 0 & 5 & 0 & 0 & & \\
\hline
\end{tabular}

$\left(\frac{1,1)(1 \mathrm{x} x)}{}\right.$ 
TABLE III

EXPLANATION OF ENTRIES IN THE INTERACTION

MATRICES, (ROUND 1)

Element Interaction Explanation

$(\mathrm{TxR})$

$(1,1)$

$(1,2)$

$(1,3)$

$(1,4)$

$(1,5)-$

$(1,9)$

$(1,10)-$

$(1,11)$

$(2,1)-$

$(2,2)$

$(2,3)$

$(2,4)$,

$(2,9)$

$(2,10)$

$(2,11)$

$(3,1)-$

$(3,11)$
1 Hardware requires the use of some $R \& D$ Base, especially a development base. In the long run, some development activities seem necessary. For instance, Japan's andi Israei's case $[93, \mathrm{p} .70 ; 67, \mathrm{pp} .56-65]$.

$1 \quad$ Self explanatory.

1 Tools and equipment would be required to manufacture hardware (and peripherals).

1 Market availability is important requirement for all three CT attributes $[67, p .38$; reference 50 is devoted entirely to CT markets].

$1 \quad$ All five human resources are necessary for the three CT attributes [18. Chapter 17;67, p.96; 93, pp. 115-1221.

1 Hardware manufacturing would require raw materials and energy.

Same as $(1,1)$ and $(1,2)$.

Software does not appear to be much dependent on tools and equipment.

1 Same as $(1,1)-(1,9)$.

0 Software does not seem to depend so much on raw materials or energy.

1 Same as $(1,1)-(1,11)$. 
(T×M)

$$
(1,1) \text { - }
$$$$
(1,4)
$$

$(3,1)$ -

$(3,4)$

$(1,5)$,

$(1,6)$

$(2,2)$,

$(2,3)$,

$(2,5)$,

$(2,6)$

$(2,1)$,

$(2,4)$

$(3,5)$ -

$(3,6)$

( $\mathrm{TxC}$

$(1,1)$,

$(1,2)$,

$(3,1)$,

$(3,2)$

$(1,3)$,

$(2,3)$

$(3,3)$

$(1,4)$

$(2,3)$,

$(3,4)$

$(1,5)$,

$(3,5)$
1

Hardware has been transferred using the channels of military, licensing and direct investment by business and government. [67, pF.44-49, pp.76-89; 93, Chapters IV and V; $71 ; 18$, p. $120 ; 98 \mathrm{~J}$.

0

Entrepreneur and journals have not been very useful in the past since the efforts involved are substantial and the scope of transfer can be quite large (e.g. discussion in 15).

1 Software seems to be best transferred by the channels of direct investment, entrepreneurs and journals [67,pp.65-88; 93,pp.106-114;79] .

0 It seems that these channels have not been employed for software in an effective manner [93, Chapter V; 15].

0

Same as $(1,5)-(1,6)$

0

Hardware, although required as part of the total CT, cannot directly improve medical care or raise the extent of literacy. Same for peripherals.

0 Basic social organization being close family units, is unlikely to be directly affected by the three CT attributes.

Same as $(1,3),(2,3)$ and $(\overline{3}, 3)$.

0

Hardware and peripherals are not likely to affect directly the size of agricultural sector 
TABLE III continued

\begin{tabular}{|c|c|c|}
\hline$(2,5)$ & 1 & $\begin{array}{l}\text { Appiication software can affect the size of } \\
\text { agricultural sector through modeling, simula- } \\
\text { tion and aid to weather forecasting }[65, \text { pp.175- } \\
182 ; 18, \text { Chapter } 15] \text {. }\end{array}$ \\
\hline $\begin{array}{l}(1,6) \\
(2,6) \\
(3,6)\end{array}$ & 0 & $\begin{array}{l}\text { It is doubtful that } C T \text { alone can improve the } \\
\text { modernization outlook of Indian Society [e.g. } \\
\text { the discussion in } 18, \text { Part V]. }\end{array}$ \\
\hline $\begin{array}{l}(1,7) \\
(2,7) \\
(3,7)\end{array}$ & 0 & $\begin{array}{l}\text { Reference } 18 \text {, Chapter } 17 \text { presents a strong } \\
\text { case that } \mathrm{CT} \text { cannot adversely affect unemploy- } \\
\text { ment. }\end{array}$ \\
\hline $\begin{array}{l}(1,8) \\
(3,8)\end{array}$ & 0 & Same as $(1,5)$ \\
\hline$(2,8)$ & 1 & Same as $(2,5)$ \\
\hline $\begin{array}{l}(1,9) \\
(1,10)\end{array}$ & 1 & CT could affect GNP $[67, \mathrm{pp} .49-54 ; 18, \mathrm{p} .122]$ \\
\hline $\begin{array}{l}(2,9) \\
(2,10)\end{array}$ & 1 & Same as $(1,9),(1,10)$ \\
\hline $\begin{array}{l}(3,9) \\
(3,10)\end{array}$ & 1 & Same as $(1,9),(1,10)$ \\
\hline $\begin{array}{l}(1,11), \\
(2,11), \\
(3,11), \\
(1,13), \\
(2,13), \\
(3,13)\end{array}$ & 0 & $\begin{array}{l}\text { No direct relationship could be found in the } \\
\text { literature, such as } 2 \text {, between gross invest- } \\
\text { ment rate, improvement or human resources } \\
\text { and the three components of } C T \text {. }\end{array}$ \\
\hline $\begin{array}{l}(1,12) \\
(2,12) \\
(3,12)\end{array}$ & 1 & CT can modernize industries [67,pp. 24-29]. \\
\hline $\begin{array}{l}(1,14) \\
(2,14) \\
(2,15)\end{array}$ & 1 & $\begin{array}{l}\text { Introduction of CT can stimulate } R \& D \text { activities } \\
{[67, \text { pp. } 56-65 ; 18 \text {, Chapters } 15,16]}\end{array}$ \\
\hline $\begin{array}{l}(1,15) \\
(2,15) \\
(3,15)\end{array}$ & 1 & $\begin{array}{l}\text { Degree of Administrative efficiency can be } \\
\text { improved by CT }[67, \text { p. 29; } 91, \text { pp.123-129]. }\end{array}$ \\
\hline
\end{tabular}


$\underline{(\mathrm{R} \times R)}$

$(1,2)$,

$(1,3)$

$(1,4)$

$(1,5)$,

$(1,9)$

$(1,10)$,

$(1,11)$

$(2,4)$,

$(2,8)$,

$(2,9)$

All others

in row 2

$(3,1)$,

$(3,4)$,

$(3,9)$

All others

in row 3

$(4,8)$

$(4,9)$

All others

in row 4

$(5,8)$

$(5,9)$

All others in row 5
1

It is rather obvious that the $R \& D$ base in turn requires capital. For instance, U.S. government's $R \& D$ activities. $R \& D$ base also requires tools and equipment.

0 Markets for CT R\&D base does not seem to be a necessary requirement $[\mathrm{e} . \mathrm{g}$. the discussion in reference 85].

1 R\&D base is heavily dependent on the avail ability and quality of the human resources [18,pp.5-6;65,pp.58-64; pp.56-64 and 96-107].

1 R\&D base would require raw materials and energy.

Capital requirements seem to be related mainly to markets, entrepreneurial and managerial skills [65,pp. 117-123].

1 All other variables do not seem to contribute directly to capital.

Tools and equipment require capital to purchase them and human resources, raw materials and energy to generate them. Other variables do not seem to be directly related.

It appears that exploitation of the CT market requires the availability of managerial and entrepreneurial skills [e.g. 67,p.96].

0 Do not seem to be directly related.

1 It also appears that managerial and entrepreneurial skills are important in improving labor productivity [18, Chapters 26-28].

0 Do not seem to be directly related. 
$(6,2)$

$(6,4)$ -

$(6,8)$

All others in row 6

$(7,1)$, $(7,2)$

All others in row 7

$(8,2)$

$(8,4)$

All others

in row 8

$(9,2)$,

$(9,4)$

All others in row 9

$(10,2)$, $(10,7)$

All others in row 10

$(11,2)$, $(11,7)$

All others in row 11
1

Labor force for CT seems to be dependent on capital available, markets, size and growth, labor productivity, technical and managerial skills [67,pp.56-64; 93,pp.115-122; 18, Chapter 15].

$0 \quad$ Do not seem to be related directiy.

Availability of a vital $R \& D$ base and capital seem necessary for maintaining technical and scientific skills for CT [e.g. see the discussion in references 96,pp.119-136 and 67, pp.56-64].

$0 \quad$ Do not seem to be related directly.

1 Again, the availability of markets and capital resources seem necessary to foster managerial skills $[67, \mathrm{pp} .96-116 ; 65, \mathrm{p} .73-76]$.

$0 \quad$ Do not seem to be related directly.

1 Same as above

0 Do not seem to be related directly.

The availability of raw materials for CT would be dependent on the availability of capital, technical and scientific skills

0 Do not seem to be related directly.

i Same as above

0 Do not seem to be related directly. 
$(\mathrm{MxM})$

$(1,1)-$

$(1,6)$

$(2,1)-$

$(2,6)$

$(3,1)$,

$(3,2)$,

$(3,6)$

$(3,4)$,

$(3,5)$

1

$(4,5)$

All others

in row 4 .

$(5,1)-$

$(5,6)$

$(6,1)-$

$(6,6)$

(CxC)

$(1,7)$

$(1,8)$,

$(1,13)$

All other

elements in

row 1
1 Military channel can contribute and/or use all channels for transferring CT; Analogy -- U.S. military [86].

I The channel -- direct investment by government_- can also utilize all other channels of transfer [89, Part III].

0 For India, it does not seem likely that the channel -- direct investment by busines - can use effectively any of the government channels for transfer of CT [18, Chapters 6,7].

Entrepreneurs and licensing arrangements can aid the direct investment by business $[89$, pp. 33-35].

1 Entrepreneur can contribute to licensing agreements for CT transier [17;89, pp.96-99].

Do not seem to be related directly.

The scope and efforts required for CT transfer to India seems to be too large for effective contribution by a single entrepreneur or journal.

It seems that medical care and unemployment are not directly related.

$1 \quad$ Better medical care in rural India can directly affect the food production and hence food/ capita and can also improve the human resources $[65, \mathrm{pp} \cdot 153-161]$.

$0 \quad$ Previous studies [2] $]^{4}$ justify the absence of relationship between the rest of the variables.

4. The entire contents of reference 2 are relevant here; in particular, Chapters IV-VIII and appendices Al-A4. 
$(2,1)$,

$(2,2)$

$(2,3)$,

$(2,4)$,

$(2,5)$

$(2,6)$

$(2,7)$,

$(2,8)$

$(2,9)$,

$(2,10)$

$(2,11)$,

$(2,12)$,

$(2,14)$,

$(2,15)$

$(2,13)$

$(3,5)$

elements

of row 3
0

1

1

0

1

0

1

1

0

$(4,2)$,

$(4,3)$,

$(4,12)$
Various studies on India indicate that these two variables are not directly related. For instance, see reference 65, pp.153-168.

Reference 2 helps justify the existence of these reiationships.

Extent of literacy can likely impact the moderni zation outlook by modifying biases and attitubes $[2 ; 96$, p.215].

Neither unemployment nor food/capita have been shown to be related to the extent of literacy. For instance, the province of Kerala in India has the highest rate of literacy and unemployment $[18, \mathrm{p} .210]$.

Extent of literacy can be correlated to GNP [23,pp.112-119 and pp.179-184].

Based on reference 2 (see footnote 4 ).

Self explanatory.

Character of basic social organization of India can significantly impact the size of agricultural sector. Close family ties have prevented the break-up of rural life in India [77,pp.19-20, 92-93].

Change in basic character of social organization is not likely to affect medical care, unemployment and food/capita, as shown by many studies on India [32,pp.260-265]. The specification for the remaining elements in row 3 are based on reference 2 (see footnote 4 ).

1 Mass communication can affect social organization structure through the diffusion of ideas and modern technology. It can also improve the extent of literacy [18,pp.84-85]. Mass 
All other elements in row 4
$(5, i)$
$(5,2)$
$(5,3)$,
$(5,6)$,
$(5,13)$
$(5,7)$,
$(5,8)$

$(5,9)$,

$(5,10)$

$(5,11)$

$(5,12)$,

$(5,14)$,

$(5,15)$

$(6,1)$

$(6,2)$

$(6,3)$

$(6,4)$,

$(6,5)$

$(6,7)$

$(6,8)$ communication can affect modernization of industry $[65, \mathrm{pp} .117-123 ; 18, \mathrm{pp} .84-85]$.

0 It seems unlikely that mass communication can directly improve medical care $(4,1)$, or affect unemployment $(4,7)$ or increase food/capita $(4,8)$. The rest of the specifications are based on reference 2 (see footnote 4 ).

$0 \quad$ Seli explanatory

1 Based on reference 2 (s ee footnote 4).

1 Change in the size of agricultural sector can affect both unemployment and food/capita by shifting labor and population patterns [32, pp. 215-234; 260-265].

1 India's GNP is quite dependent on agriculture.

$0 \quad$ Based on reference 2 (see footnote 4 ).

0 It seems unlikely that modernization outlook can solely improve medical care.

1 Modernization outlook emphasizes education [23, Chapters VI and VII].

0 Based on reference 2 (see footnote 4 ).

1 Based on reference 2 (s ee footnote 4).

0 It appears that modernization outlook does not affect directly unemployment or foot/capita. UK is an example [e.g. 18, pp.31-32, 135]. 

$(6,9)$
$(6,10)$
$(6,11)$,
$(6,13)$,
$(6,14)$
$(6,12)$,
$(6,15)$
$(7,1)$,
$(7,2)$
0 Surprisingly, GNP cannot be correlated to modernization uutlook of society; e.g., Japan's case $[88,2]$.
0 Based on reference 2 (see footnote 4 ).
1 Based on reference 2(see footnote 4 ).
C It appears that unemployment cannot be related directly to the extent of literacy or medical care; e.g. Kerala's case cited in the element $(2,7)$.

$(7,4)$

$(7,5)$

$(7,6)$

$(7,8)$

$(7,9)$,

$(7,10)$

$(7,11)$,

$(7,12)$

$(7,13)$

$(7,14)$,

$(7,15)$

0 It seems unlikely that unemployment can directly increase/decrease the extent of mass communication (such as radio, TV, newspapers/ 1000 population).

$0 \quad$ Agricultural sector seems to depend more on economic and meterological factors.

0 Modernization outlook depends more on variables such as extent of literacy [2].

0 Unemployment not directly related to food/ capita [99, pp. 258-266].

$1 \quad$ Self-explanatory

$0 \quad$ Based on references: 32, p.127; 42, pp.959-977.

1 Unemployment could decelerate the rate of improvement of human resources [18, Chapter 26].

$0 \quad$ Based on references: 18, pp. 5-6; 77, pp.35-37. 
$(8,9)$,

$(8,10)$

$(8,13)$

All others in row 8

$(9,3)$

All others in row 9

$(10,3)$

All others in row 10

$(11,1)$,

0

$(11,3)-$

$(11,6)$

$(11,7)$

$(11,8)$

$(11,9)$,

$(11,10)$

$(11,11)-$

$(11,15)$

$(12,1)$,

$(12,2)$

$(12,3)$, $(12,4)$
1 It appears that the change in food/capita can affect GNP, GNP/capita and the improvement of human resources [23,pp.29-46]. All other entries in this row are zero, based mainly on reference 2 (see footnote 4 ).

$0 \quad$ Do not seem to be directly related.

o Same as above

1 Except the character of basic social organization, GNP is likely to affect all other elements and row 9 directly $[2 ; 42$, Appendix VIII].

$0 \quad$ Same as above

$1 \quad$ Same as above

GIR (Gross Investment Rate) does not seem to affect directly medical care or extent of literacy $[2$, see footnote 4$]$.

$0 \quad$ Based on reference 2 (see footnote 4).

1 GIR can increase/decrease unemployment [18, Chapter 26; 32 pp.182-183].

0 It appears that food/capita is not directly related to GIR.

1 It is rather obvious that GIR can affect GNP and GNP/Capita .

Based on reference 2 (see footnote 4)

Same as $(11,1),(11,2)$.

$0 \quad$ Based on reference 2 (see footnote 4) 
$(12,5)$,

$(12,6)$

$(12,7)$,

$(12,8)$

$(12,9)-$

$(12,11)$

$(12,13)$,

$(12,14)$

$(12,15)$

$(13,1)$,

$(13,2)$

$(13,3)$,

$(13,4)$

$(13,5)$

$(13,6)$

$(13,7)$

$(13,8)$

$(13,9)$,

$(13,10)$

$(13,11)-$

$(13,15)$

$(14,11)$

All others

in row 14
1

0

1

1

0

1

0

1

0

1

0

1

0

1

$\checkmark$

Based on reference 2 (see footnote 4)

Based on references: 18, pp.24-25; 42 , pp. $693-703$.

Based on reference 2 (see footnote 4 ).

Based on references: 65, pp.65-73; 67,pp. 56-58.

Based on reference 2 (see footnote 4 ).

It seems likely that the improvement of human resources (e.g., doctors) can leau to better medical care and extent of literacy.

Based on references: 2 (see footnote 4); 61 .

Size of agricultural sector can be influenced by improving human resources [65,pp.179-181].

Based on reference 2 (see footnote 4 ).

Improvement of human resources can shift the employment patterns.

Food/capita seems to be dependent more on variables such as agricultural sector.

Based on references 2 (see footnote 4) and 61 , pp. $112-119$.

Based on references 2 (see footnote 4 ).

Increased $R \& D$ expenditures, especially in India, can affect modernization of industry $[96, \mathrm{pp} .16-18]$.

Studies, such as Project Hindisght [41,65, pp. 126-140], show that $R \& D$ expenditures by 
themselves cannot affect directly the other elements of row 14 .

$(15,6)$

All others in row 15
$(1,12)$

$(1,14)$

All others in row 1

$(2,9)-$

$(2,11)$,

$(2,14)$

All others in row 2

$(3,12)$,

$(3,13)$

All others in row 3

$(4,7)$

$(4,11)$,

$(4,14)$

All others in row 4
Based on reference 2 (see footnote 4 )

Based on reference 2 and other studies, such as 101, Chapter 1, which indicate that the improvement in the degree of administrative efficiency does not seem to be correlated to the other consequences of CT.

The size and the availability of $R \& D$ base can influence modernization of industry and $R \& D$ expenditures $[96, \mathrm{pp}, 16-18]$.

0 Other variables do not seem to be affected directly $[2 ; 18$, Part V].

1 The resource of capital seems to affect directly the economic variables, GNP, GNP/capita, GIR and $R \& D$ expenditures.

0 For the remaining elements of row 2 , the effect seems to be indirect.

Availability of better tonls and equipment can affect the modernization of industry and improve human skills. All other variables seem to be indirectly affected.

A large market for $\mathrm{CT}$ can affect unemployment [18, Chapter 17].

1 Markets for CT-size and growth-can affect the GIR and the money available for $R \& D$ expenditures $[67, \mathrm{pp} .38-43,49-54]$.

0 It seems that the CT markets by themselves would not affect directly the sociocultural variables. The economic variables, such as GNP component of $\mathrm{CT}$, also seem to have an indirect effect $[\mathrm{e} . \mathrm{g} ., 93, \mathrm{pp} .123-140]$. 
$(5,1)-$

$(5,10)$,

$(5,14)$,

$(5,15)$

$(5,11)-$

$(5,13)$

$(6,7)$,

$(6,12)$

$(6,13)$

All others

in row 6

$(7,6)$,

$(7,12)$,

$(7,14)$

$(7,15)$

All others

in row 7

$(8,6)$,

$(8,12)$,

$(8,15)$

All others

in row 8

All entries

of row 9

All entries of rows 10 , 11
0
Labor productivity does not appear to directly affect the sociocultural variables. It seems to have major impact on the economic variables, such as modernization of industry, GIR and improvement of human resources $[66 ; 15 ; 18$, Chapter 26].

The size of the labor force for CT can affect unemployment patterns and modernization of of Indian industries. The effect on sociocultural variables and other economic variables appears to be small $[15 ; 18$, Chapter 17].

Technical and scientific skills required for $C T$ can change modernization outiook, $R \& D$ expendi tures, human resource improvement and administrative efficiency. It appears that sociocultural variables are not likely to be impacted directly [96,pp.62-64 and 184-213; 93pp.115-123].

Management skills can affect the economic variables: GIR, level of modernization of industry, $R \& D$ expenditures and the degree of administrative efficiency. [67,pp.96-107]. Its influence on other variables in row 8 seems doubtful and at best seems to be indirect [for instance, see the discussion in 40, pp.63-76].

It appears that the entrepreneurial skills by themselves are not likely to impact any of the selected variables directly, because of the scope of the effort involved.

From the studies on CT, such as $18-p, 120,79$, $83,91-$ pp. $70-73$, and 102, it appears that raw materials and energy requirements of $\mathrm{CT}$ are modest and therefore, would not impact directly the variables in rows 10 and 11 . 


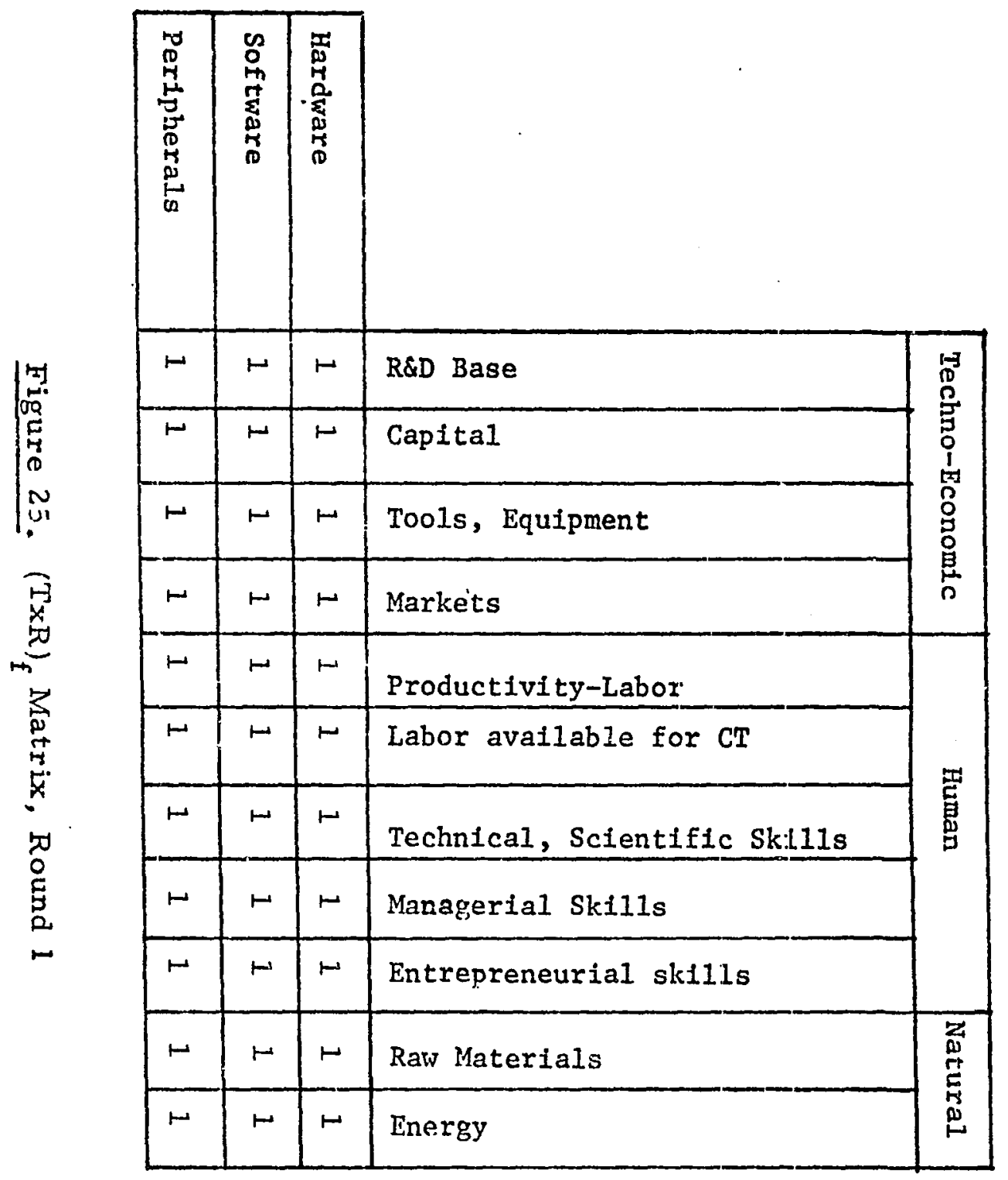




\begin{tabular}{|c|c|c|c|c|c|c|}
\hline & \multicolumn{2}{|c|}{ Government } & \multicolumn{3}{|c|}{ Business } & \multirow{2}{*}{ 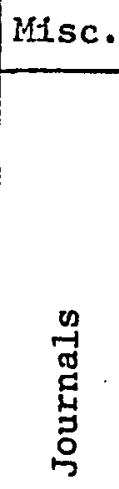 } \\
\hline & 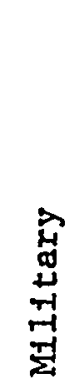 & 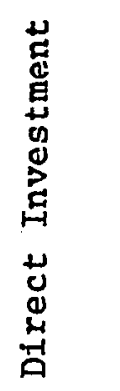 & 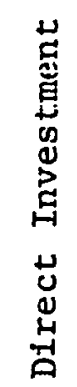 & 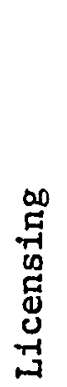 & 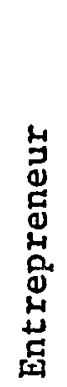 & \\
\hline Hardware & 1 & 1 & 1 & 1 & 1 & 1 \\
\hline Sof tware & 1 & 0 & 1 & 1 & 1 & 1 \\
\hline Peripherals & 1 & 1 & 1 & 1 & 1 & 1 \\
\hline
\end{tabular}

Figure 26. $(\mathrm{TxM})_{\mathrm{f}}$ Matrix, Round 1 


\begin{tabular}{|c|c|c|c|c|}
\hline 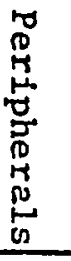 & 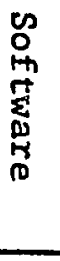 & 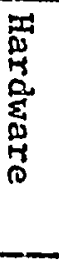 & . & \\
\hline$\mapsto$ & $\omega$ & س & Quality of Medical Care! & 营 \\
\hline$\mapsto$ & $\mu$ & $\mapsto$ & Extent of Literacy & 国 \\
\hline$\omega$ & $\mapsto$ & $\omega$ & Char. of Basic Soc. Org. & \multirow{4}{*}{ 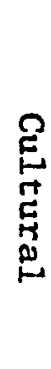 } \\
\hline$\mapsto$ & $\vdash$ & $\mapsto$ & Extent of Mass Communication & \\
\hline$\mapsto$ & $\mapsto$ & س & Size of agricultural sectior & \\
\hline$\mapsto$ & $\omega$ & $\mapsto$ & Degree of Modernization Outlook & \\
\hline$\mapsto$ & $\mapsto$ & $\mapsto$ & Unemployment & \multirow{2}{*}{ \& } \\
\hline$\mapsto$ & $\omega$ & $\mapsto$ & Food/cap1ta, Calorie Intake & \\
\hline$\mapsto$ & $\mapsto$ & $\mapsto$ & GNP Component of CT & \multirow{7}{*}{ 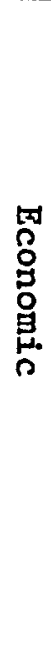 } \\
\hline$\mapsto$ & $\mapsto$ & $\sim$ & GNP/capita change due to CT & \\
\hline$\mapsto$ & $\mapsto$ & $\mapsto$ & Gross Investment Rate & \\
\hline$\vdash$ & 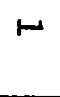 & $\omega$ & Level of Modernization of Ind. & \\
\hline$\mapsto$ & $\mapsto$ & $\mapsto$ & $\begin{array}{l}\text { Rate of Improvement of Human } \\
\text { Resources }\end{array}$ & \\
\hline$\mapsto$ & $\mapsto$ & $\omega$ & R\&D Expenditures & \\
\hline$\mapsto$ & $\mu$ & $\mapsto$ & Degree of Admin. Efficiency & \\
\hline
\end{tabular}




\subsection{Analysis and Commerts on the Synthesized Matrices of Round 1}

The $(T x R)_{f}$ matrix is as expected. Since one of the criteria for selecting resource variables was relevancy, it is not surprising to observe that only $l^{\prime}$ 's appear in the $(T \times R)_{f}$ matrix. It is interesting to note, however, that all elements of the $(T \times R)$ and $(R x R)$ matrices are not l's. Therefore, the resulting $l^{\prime} s$ in the $(T \times R)_{f}$ matrix are due to indirect paths through self-interaction.

The $(T \times M)_{f}$ matrix represents results that were non-obvious at first, since the matrix (TxM) contained several zero entries indicating nonexistence of interactions between the variables. The predominance of $1^{\prime} s$ in the $(T \times M)_{f}$ matrix seems mainly due to the overlap between the transfer mechanisms.

The $(\mathrm{TxC})_{f}$ matrix also represents results that were non-obvious at first, since compared to the number of zeroes in the ( $\mathrm{T} x \mathrm{C})$ matrix, the synthesized $(\mathrm{TxC})_{f}$ matrix consists of all l's. This result is mainly due to the inclusion of GNP and GNP/capita as economic variables. It so happens that GNP interacts with most variables, thus producing indirect paths leading to $\mathrm{l}^{\prime} \mathrm{s}$ in the $(\mathrm{T} \times \mathrm{C})_{\mathrm{f}}$ matrix.

The analysis of the first round development of Level 2 led to a reexamination of the various interactions. It was observed that some of the variables overlap while others are not as significant. Hence, these variables can be combined into one variable, or dropped. For instance, the $(\mathrm{TxR})_{f}$ matrix indicated that the energy i cquirement for $\mathrm{CT}$ is quite modest and does not affect other variables (such as 
consequences), and hence, may not be considered further. The variables GNP and GNP/capita also seem to be of little help in the analysis because: (a) they predominate many other variables; hence, their interaction with other variables is obvious and can be preassessed, and (b) magnitude of CT contribution to India's GNP is quite difficult to estimate at the present time, although such information would be useful. The resource variables - - managerial skills and entrepreneurial skills-overlap significantly, at least for the CT transfer. Thus, they can be combined into a single variable. The list of transfer mechanisms remains unchanged.

\subsection{Development of Levels 1 and 2: (Round 2)}

Based on the review of the Round 1 matrices and the above comments, the Level 1 structure was revised. The corresponding morphological tree is shown in Figure 28. It can be noticed that the number of resource variables are reduced from 11 to 9 , and the impact variables from 15 to 13 .

The revised static structure is used to develop a new set of interaction matrices. Most entries in these matrices follow from the reasons cited earlier and in Table III. A few interactions, however, have been revised in light of the comments made above. These revised interactions are explained in Table IV. The seven interaction matrices developed in round 2 are shown in Figure $29(\mathrm{a})-(\mathrm{g})$. These matrices are synthesized to yield the $(T \times R)_{f},(T \times M)_{f}$, and $(T x C)_{f}$ matrices 


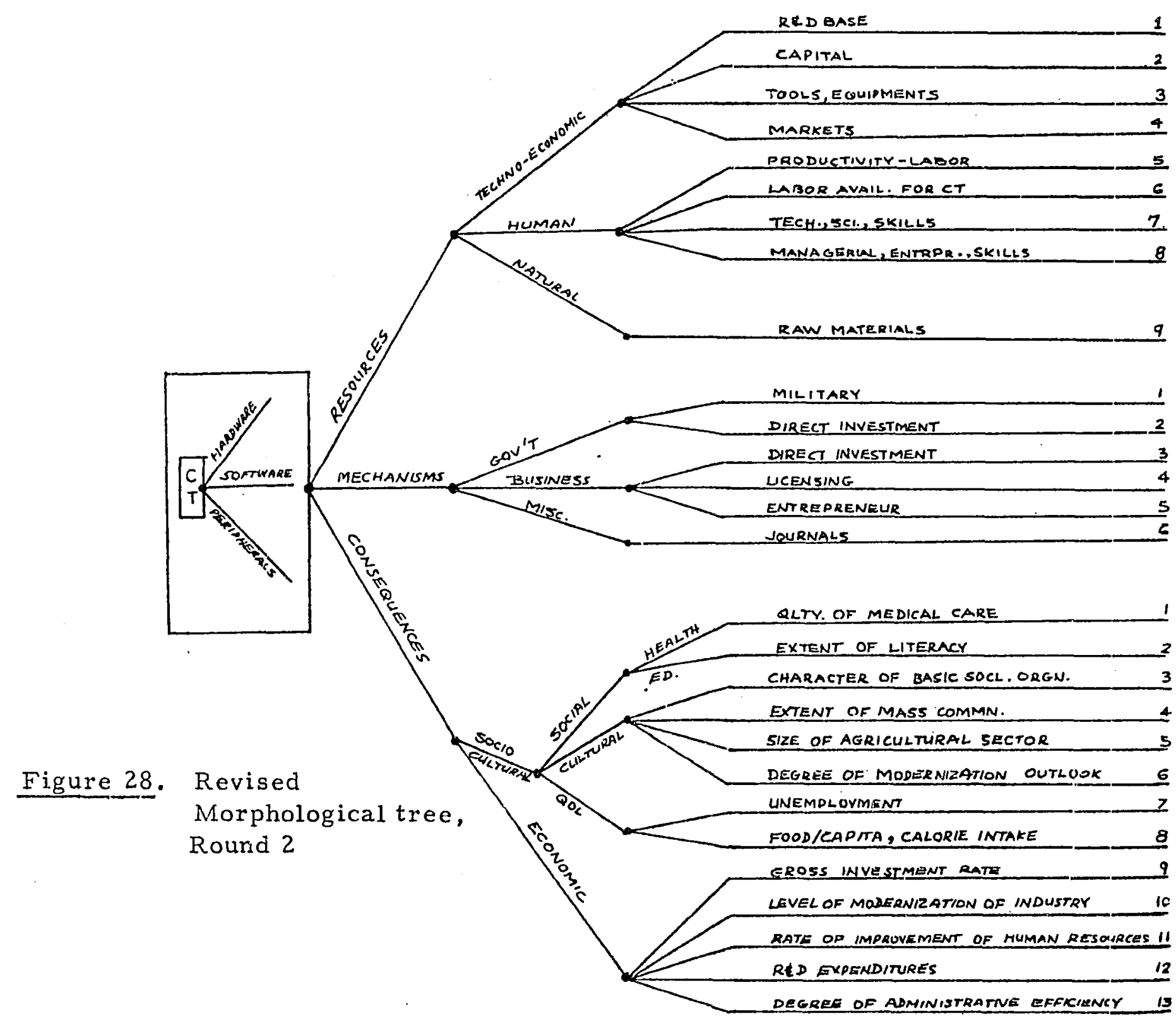



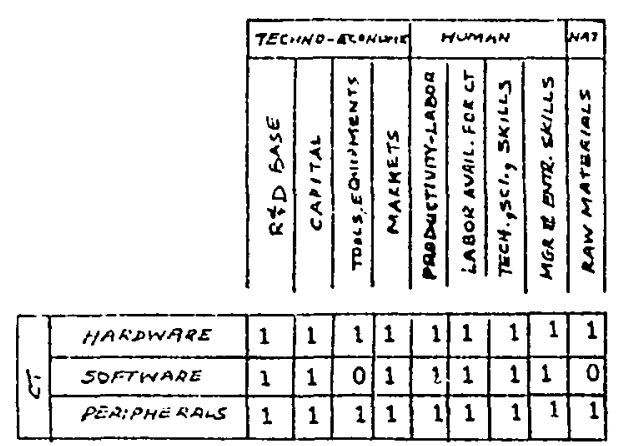

(a) ( $\mathrm{P} \times \mathrm{R})$

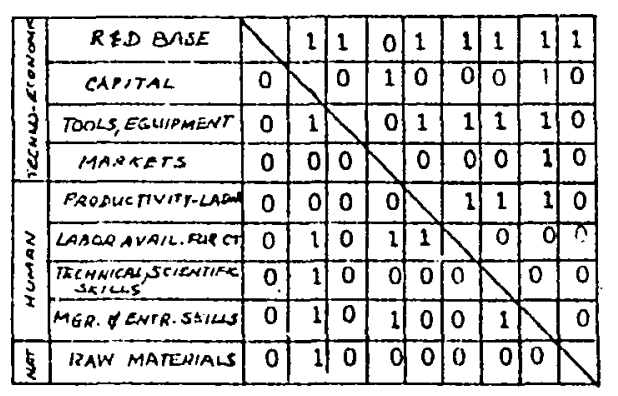

(d) $($ rixR)

Figure 29. Interaction Matrices, Round 2
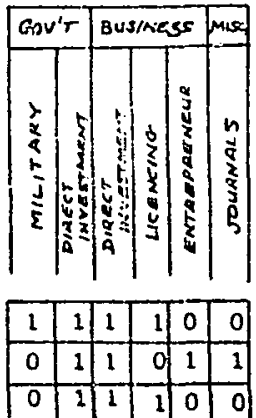

(b) (1'XM)

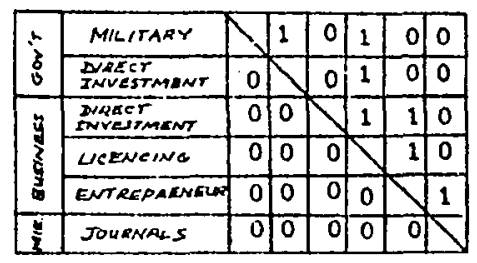

(e) $(\mathrm{s} \times \mathrm{Ni})$

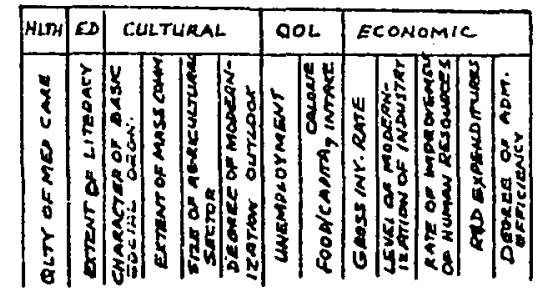

\begin{tabular}{|l|l|l|l|l|l|l|l|l|l|l|l|l|}
\hline 0 & 0 & 0 & 0 & 0 & 0 & 0 & 0 & 0 & 1 & 0 & 1 & 1 \\
\hline
\end{tabular}

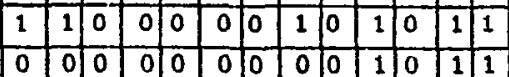

(c) $(\mathrm{TxC})$

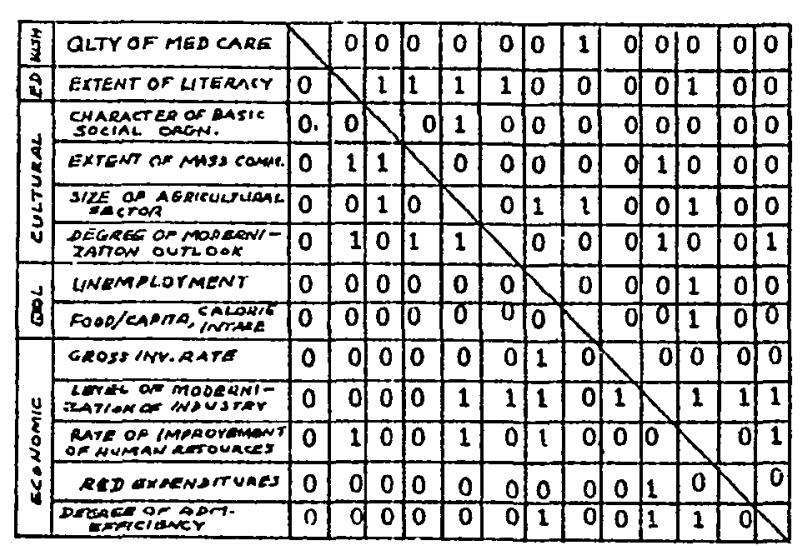

(f) $(C x C)$

\begin{tabular}{|c|c|c|c|c|c|c|c|c|c|c|c|c|}
\hline & RED BASE & 0 & q & 0 & of & do & & & 1 & & 10 & 0 \\
\hline & camital & 0 & 90 & 0 & 0 & a & & 91 & 0 & & $\sqrt{0}$ & 0 \\
\hline & Tooks, EQupMant & 0 & 90 & 0 & 0 & do & & 0.0 & 0 & & 0 & $\tilde{0}$ \\
\hline & MARKAT TS & 0 & 0 & 0 & 0 & di & & 01 & 10 & $\overline{0}$ & 10 & 0 \\
\hline \multirow{4}{*}{8} & proxetrirn-Lasese & 0 & do & 0 & 0 & 0 & & 91 & To & $\overline{0}$ & 0 & 0 \\
\hline & LAOOP AVAK. FORCT & 0 & do & 0 & 0 & & 0 & do & 10 & 0 & 0 & \\
\hline & 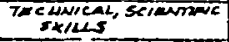 & 0 & ఏ0 & 0 & 0 & & 0 & 00 & 1 & 0 & 1 & 0 \\
\hline & AGR. IEVTR. Sk/LES & of & 90 & 0 & 이 & & 0 & 90 & 1 & & 0 & 1 \\
\hline $\mathbf{s}$ & RAW MATERTALS & o & do & 0 & 이 & & 0 & 00 & 0 & & 0 & 0 \\
\hline
\end{tabular}

(x) $(n \times C)$ 
TABLE IV

REVISED INTERACTIONS FOR ROUND 2

Matrix Revision

$(\mathrm{TxR}) \quad$ Norie

( TxM) The element $(3,1)$ changed to zero, since it seemed that the military channel does not depend much on peripheral technology $[93, \mathrm{pp} .140-150]$

( TxC) The element $(2,5)$ changed to zero as it is felt that sofit ware affects agricultural productivity more than its size $[18, \mathrm{p} .103]$

$(R \times R) \quad A$ large $R \& D$ base does not seem mandatory for the avail ability of technical and scientific skills, since a substantial $R \& D$ base in other fields, such as atomic energy, exists; hence, the element $(7,1)$ changed to zero.

$(\mathrm{M} \times \mathrm{M})$ In India, the direct investment by government in massive industries is not influenced much by the performance of military channel $[52, \mathrm{pp} .38-39]$; hence $(1,3)$ changed to zero. Again, the channel of military does not seem to depend much on journals or entrepreneurs. Hence, $(1,5)$, $(1,6)$ changed to zero. $(2,1),(2,5)$ and $(2,6)$ were changed to zero for the same reasons as $(1,3)$ and $(1,5)$ and $(1,6)$. $(5,6)$ is changed to 1 , since the entrepreneur can utilize information available in journal such as trade magazines. 
(CxC) Further literature search revealed that the quality of medical care and the rate of improvement of human resources do not saem to be directly correlated [e.g. 23, Chapter XII]. In past, change in the size of agricultural structure has not appeared to impact the modernization outlook. Hence, $(5,6)$ was changed to zero. The current and past unemployment situation in India seems not to significantly affect the character of basic social organization. Hence, $(7,3)$ changed to zero. The level of modernization of industry can likely impact unemployment and degree of adminis trative efficiency by changing employment patterns. Hence, the entries $(10,7)$, $(10,13)$ changed to 1 . The impact of rate of improvement of human resources on medical care is changed to zero, based on further literature search [23, Chapter XIr]. It, however, does impact the degree of administrative efficiency, since such efficiency seems to depend on the performance of human resources, Hence, $(11,15)$ changed to 1 . The degree of administrative efficiency can impact unemployment by shifting employment patterns, improving industries and human resources. Hence the entries $(13,7),(13,10),(13,11)$ are changed to 1 . However, it does not seem to impact the modernization outlook of the society. Hence $(13,6)$ changed to zero. 
TABLE IV continued

( $\mathrm{RxC}$ The element $(2,7)$ changed to zero since capital does not seem to affect unemployment directly. The elements $(3,10)$, $(3,11)$ changed to zero for the same reasons as $(2,7)$. The elements $(4,7),(4,12)$ are changed to zero for the same reasons as $(2,7)$. The elements $(5,7)$ changed to 1 since increase/decrease in productivity can affect unemployment. The element $(5,10),(5,11)$ changed to zero for the same reasons as $(2,7)$. The impact of the size of labor force for CT does not appear to impact the consequences because of the scope of the efforts involved. Hence, all entries in this row are zero. The elements $(7,6),(7,13)$ and $(8,6)$ changed to zero for the same reasons as $(2,7)$. 
shown in Figures 30-32. These matrices represent the structure of $\mathrm{TT}$ and they would be employed to analyze TT performance.

\subsection{Comments on the Synthesized Matrices (Round 2)}

All entries of $(T \times R)_{f}$ matrix again are l's. This was expected for the reasons mentioned in section 4.3 . The matrix (TxM) $f$ indicates that almost all transfer mechanisms are useful. It can be noted, however, that the military channel does not seem useful for software and peripheral technology transfer. The journals seem to be poor channels to transfer hardware and peripherals. Discussions in references 67 and 93 lend support to these statements.

The matrix $(\mathrm{TxC})_{f}$ yields interesting and nonobvious results. It is interesting to observe that India's needs are really in the software area. This observation seems correct since computer utilization, rather than manufacturing, is more useful to India. If the three CT attributes are taken together, one observes somewhat surprising results that CT has little impact on variables such as the extent of literacy, character of basic social organization, extent of mass communication and food/ capita. Yet these variables are considered important for rapid economic variables is significant. This shows that the economic viewpoint alone tells only the half-truth; that is, if the analysis was done strictly in terms of economic variables, there would be little question of importing or borrowing CT to India. However, one has second thoughts when he observes rather a small impact on such important sectors as literacy, food, basic social organization (family units) and mass communication. 


\begin{tabular}{|l|l|l|l|l|l|l|l|l|l|}
\hline \multicolumn{2}{|l|}{ Techno-Economic } & \multicolumn{3}{|c|}{ Human } \\
\hline & & & & & & & & & \\
Nat
\end{tabular}

Figure 30. (TxR) $\mathrm{f}$ Matrix, Round 2 


\begin{tabular}{|c|c|c|c|c|c|c|}
\hline & \multicolumn{2}{|c|}{ Gov't } & \multicolumn{3}{|c|}{ Business } & \multirow[b]{2}{*}{ 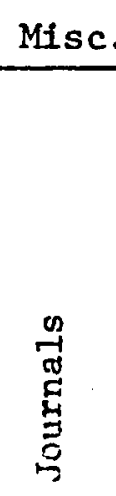 } \\
\hline & 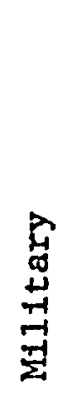 & 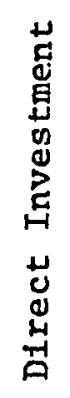 & 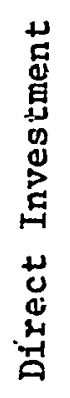 & 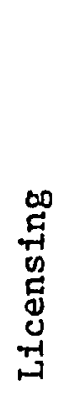 & 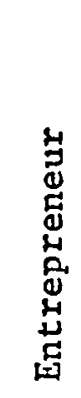 & \\
\hline Hardware & 1 & 1 & 1 & 1 & 1 & 0 \\
\hline Sof tware & 0 & 1 & 1 & 1 & 1 & 1 \\
\hline Peripherals & 0 & 1 & 1 & 1 & $i$ & 0 \\
\hline
\end{tabular}

Figure 31. (TxM) $)_{f}$ Matrix, Round 2 


\begin{tabular}{|c|c|c|c|c|}
\hline 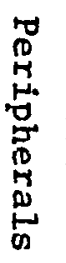 & 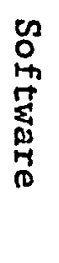 & 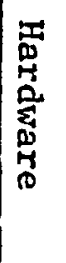 & & \\
\hline 0 & $r$ & 0 & Quality of Medical Care & 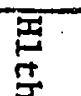 \\
\hline$\mapsto$ & $r$ & $\mapsto$ & Extent of Literacy & 牙 \\
\hline 0 & $\mapsto$ & 0 & Character of Basic Soclal Orgn. & \multirow{4}{*}{ 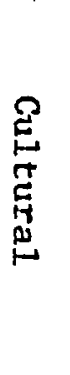 } \\
\hline 0 & 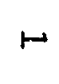 & 0 & Extent of Mass Communication & \\
\hline$\mapsto$ & 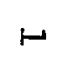 & 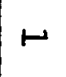 & Size of Agricuitural Sector & \\
\hline 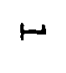 & $\mapsto$ & $\mapsto$ & Degree of Modernization Outlook & \\
\hline$\mapsto$ & $\hookleftarrow$ & $\mapsto$ & Unemployment & \multirow{2}{*}{8} \\
\hline 0 & $\hookleftarrow$ & 0 & Food/capita or Calorie Intake & \\
\hline$\mapsto$ & $\mapsto$ & $w$ & Gross Investinent Rate & \\
\hline$\mapsto$ & $\mapsto$ & $\mapsto$ & Level of Modernization of Ind. & \\
\hline$\mapsto$ & $\mapsto$ & $r$ & Rate of Improvement of Human Res & \\
\hline$\mapsto$ & $\mapsto$ & $\mapsto$ & R\&D Expenditures & \\
\hline$\vdash$ & 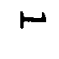 & 1 & Degree of Administrative Eff. & \\
\hline
\end{tabular}


The desirability and usefulness of a multidimensional view point of $\mathrm{TT}$ is thus evident from information outputs of the three final matrices. The fact that a strict economic viewpoint of $\mathrm{TT}$ gives an incomplete picture of the consequences of CT can be established from the $(\mathrm{TxC})_{\mathrm{f}}$ matrix.

More useful insight into planning and policymaking aspects of the transfer can be obtained by utilizing the second round $(\mathrm{TxR})_{f},(\mathrm{TxM})_{f}$ and $(\mathrm{TxC})_{f}$ matrices to develop the three performance measures -- feasibility, efficiency or applicability of transfer mechanisms, and desirability of CT transfer of India.

4.6 Performance Measures for CT Transfer to India

\subsubsection{Feasibility of CT Transfer to India}

The procedure outlined in section 3.10 .1 is used to determine the feasibility of CT transfer to India.

Since all elements of (TxR) matrix are l's, the measures availability (a), productivity (b), and cost (c) - are developed for each resource variable. These are shown in Table $\mathrm{V}$. In each column a brief statement is provided to explain why the entry is 1 or 0 . A blank entry implies that one of the three measures is not applicable.

The five policy statements $\mathrm{P}_{1}-\mathrm{P}_{5}$, discussed in section 3.10 .1 , are developed in Table VI. The results of this development can be summarized as: 


\title{
TABLE V
}

SPECIFICATIONS FOR THE MEASURES:

a-AVAILABILITY; b-PRODUCTIVITY; c-COST

\author{
Resource \\ Specification for $a, b, c$ \\ $R_{1}: R \& D$ Base $\quad a=0$ : Currently, the $R \& D$ base does not exist on the \\ scale required for $\mathrm{CT}$, bitt is potentially possible \\ $[18$, Chapter $17 ; 65, \mathrm{pp} .58-64]$. \\ $\mathrm{b}=1$ : The productivity of the $R \& D$ base, currently \\ existing in India (e.g., atomic energy establishment \\ and aircraft technology), is quite good [65, pp.58-64; \\ 96, pp. 16-18]. \\ $c=1$ : It has been estimated that the cost of developing \\ $R \& D$ base in India is quite low. $[65, p p .53-64 ; 18$, \\ pp. 15-18]. \\ $R_{2}$ : Capital $\quad a=1$ : Most of the capital needed for CT can be gener- \\ ated locally $[18$, Chapter 8$]$. \\ b : No available data and does not seem quite relevant. \\ $c=0$ : As discussed in various references, the cost of \\ capital in developing countries is quite high; e.g. , \\ reference 46 , Chapter II. \\ $\mathrm{R}_{3}$ : Tools, $\quad \mathrm{a}=1$ : Most light, heavy and processing equipments \\ Equipment and tools are available or can be manufactured in \\ India [18, Chapter 10].
}


$b=1$ : The quality of tools and equipment has proven to be $\operatorname{good}[18$, Chapter 10$]$.

$c=1$ : Since equipment is available locally, costs seems to be low.

$\mathrm{R}_{4}:$ Markets

$a=1:$ Forecasts and discussion on CT indicate that India is potentially a large market $[\mathrm{e} . \mathrm{g} ., 18$, Chapter 17].

b, c: not applicable

$R_{5}:$ Produc $-\quad a, c:$ not applicable

tivity, Labor $\quad b=0$ : Productivity of average human resource is considered low for India [23, Table IV $-3-1 ; 18$, Chapter 26].

$R_{6}:$ Labor $a=1$ : It is possible to get most labor force for CT in Available for India $[65, \mathrm{pp} \cdot 58-64]$. For instance, many Indian $\mathrm{CT}$ universities now have cúmputer science programs $[18, \mathrm{pp} .116-117]$ $b=0$ : Same as for $R_{5}$. $c=1$ : Cosí of labor in India is quite reasonable [42, pp.948-951]

$\mathrm{R}_{7}$ : Technical a=1: Proper technical and scientific skills are availScientific able in India, e.g., those for atomic energy, air Skills craft and electronics technologies [18, Chapter 17; 65, pp. 58-64]. 


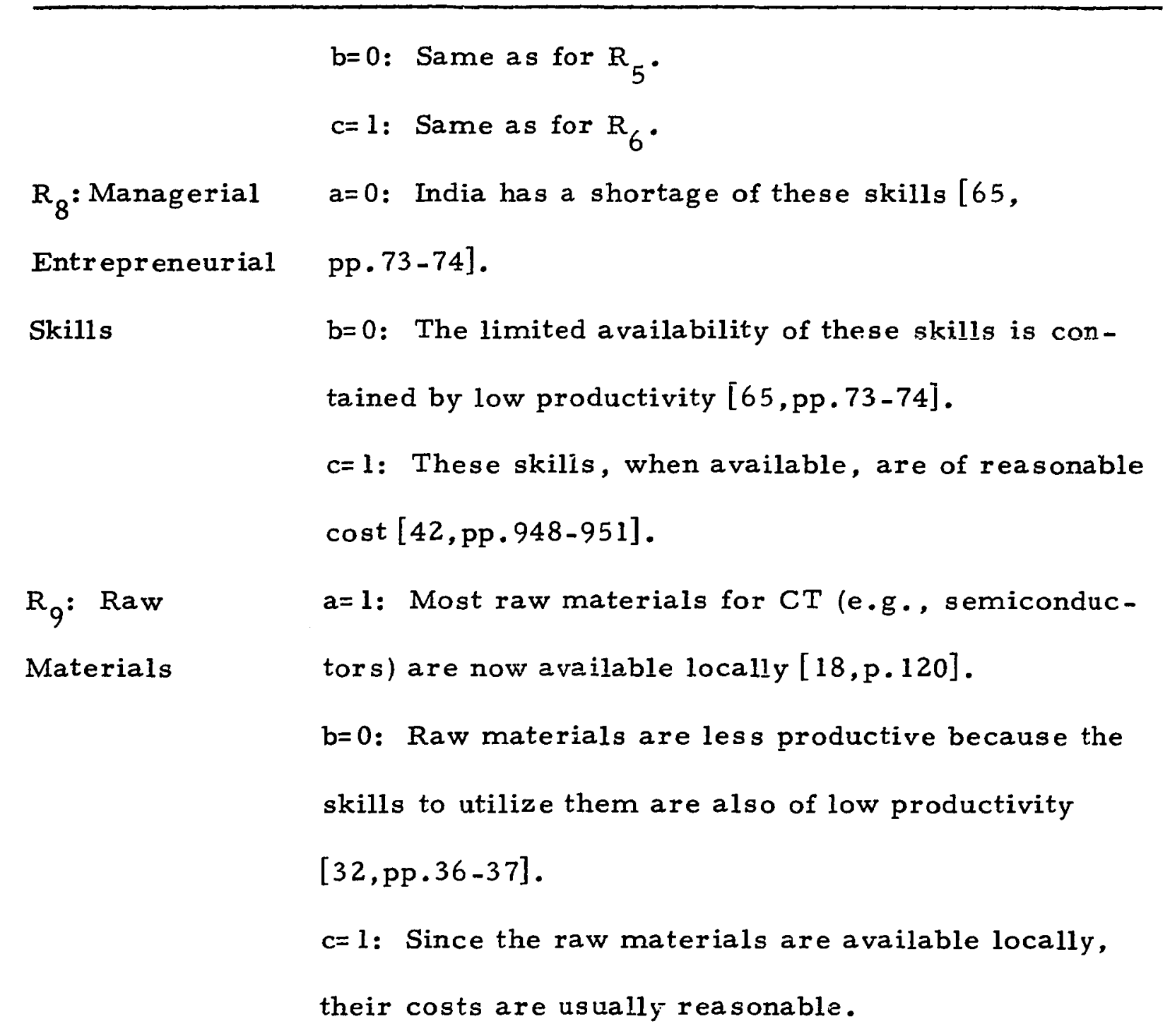


TABLE VI

POLICY STATEMENTS FOR TT FEASIBILITY

\begin{tabular}{|l|c|c|c|c|c|}
\cline { 2 - 6 } & \multicolumn{5}{|c|}{ Policy Statements } \\
\hline$R_{1}:$ R\&D Ba se & $P_{1}$ & $P_{2}$ & $P_{3}$ & $P_{4}$ & $P_{5}$ \\
\hline$R_{2}:$ Capital & 0 & 0 & 0 & 1 & 1 \\
\hline$R_{3}:$ Tools, Equip. & 0 & 1 & 0 & 0 & 1 \\
\hline$R_{4}:$ Markets & 1 & 1 & 1 & 1 & 1 \\
\hline$R_{5}:$ Labor Prod. & 1 & 1 & 1 & 1 & 1 \\
\hline$R_{6}:$ Labor for CT & 0 & 0 & 1 & 0 & 1 \\
\hline$R_{7}:$ Tech, Sci. Skills & 0 & 0 & 1 & 0 & 1 \\
\hline$R_{8}:$ Mgr . Entr. Skills & 0 & 0 & 0 & 0 & 1 \\
\hline$R_{9}:$ Raw Materials & 0 & 0 & 1 & 0 & 1 \\
\hline
\end{tabular}




$$
\begin{aligned}
& P_{1}=1, \text { for } R_{3}, R_{4} \\
& P_{2}=1 \text {, for } R_{2}, R_{3}, R_{4} \\
& P_{3}=1 \text {, for } R_{3}, R_{4}, R_{5}, R_{6}, R_{7} \\
& P_{4}=1 \text {, for } R_{1}, R_{3}, R_{4} \\
& P_{5}=1 \text {, for } R_{1}, R_{2}, R_{3}, R_{4}, R_{5}, R_{6}, R_{7}, R_{8}, R_{9}
\end{aligned}
$$

Recall that the policy $P_{1}$ implies feasibility under any conditions, while $\mathrm{P}_{5}$ is considered infeasible. It can be observed in Table $\mathrm{V}$ that a resource variable appears under more than one policy; hence, a procedure for ranking these policies is needed. For instance, the resource variable $R_{3}$ appears under policies $P_{1}, P_{2}, P_{3}, P_{4}$, and $P_{5}$. Since the policy $P_{1}$ is most desirable, $R_{3}$ should be classified under $P_{1}$ (i.e., it should be considered feasible in terms of the requirements of policy $P_{1}$ ) and hence, deleted from $\mathrm{P}_{2}-\mathrm{P}_{5}$. However, if a resource variable satisfies $\mathrm{P}_{2}-\mathrm{P}_{4}$ requirements, it will not be possible to classify it under a single policy statement unless a criterion for preferring one policy to another exists.

To develop such a criterion, recall that: $P_{l}$ implies feasibility under any conditions and without any constraints, $\mathrm{P}_{5}$ is feasible under any conditions, while $P_{2}, P_{3}, P_{4} a:=$ feasible under the constraints of cost, productivity and local availability respectively. Hence, obviously $P_{1}$ is the most preferable policy statement, $P_{5}$ is the least preferable. $\mathrm{P}_{3}$ allows low productivity. However, low productivity applies to the human resources only, as discussed in Tables II and III. The size of available labor force for $C T$ and provision of proper training facilities 
can help overcome the productivity constraint. Hence, we rank $\mathrm{P}_{3}$ as the second most preferable policy. It is difficult to rank $\mathrm{P}_{2}$ or $\mathrm{P}_{4}$ as the third most preferable policy. We chose $\mathrm{P}_{4}$ because the resources can be imported (e.g., Japan's case) but it is difficult, relatively speaking, to counter the high costs of capital. Indeed, the high cost of capital in developing countries is a major obstacle to TT. Thus, we rank the five policies in the following order of preference: $P_{1}, P_{3}, P_{4}, P_{2}, P_{5}$. It must be noted carefully that this order of preference holds only for a particular $\mathrm{TT}$ (CT in this case), and for a particular country.

A simple procedure can now be devised to classify systematically a resource variable under a single policy statement. First, a scale showing the ranks of $\mathrm{P}_{1}-\mathrm{P}_{5}$ is generated, as shown below. Under each policy statement, the resource variables having value of 1 (from Table VI) for that policy statement are listed. Nexr, begin at the left side of the

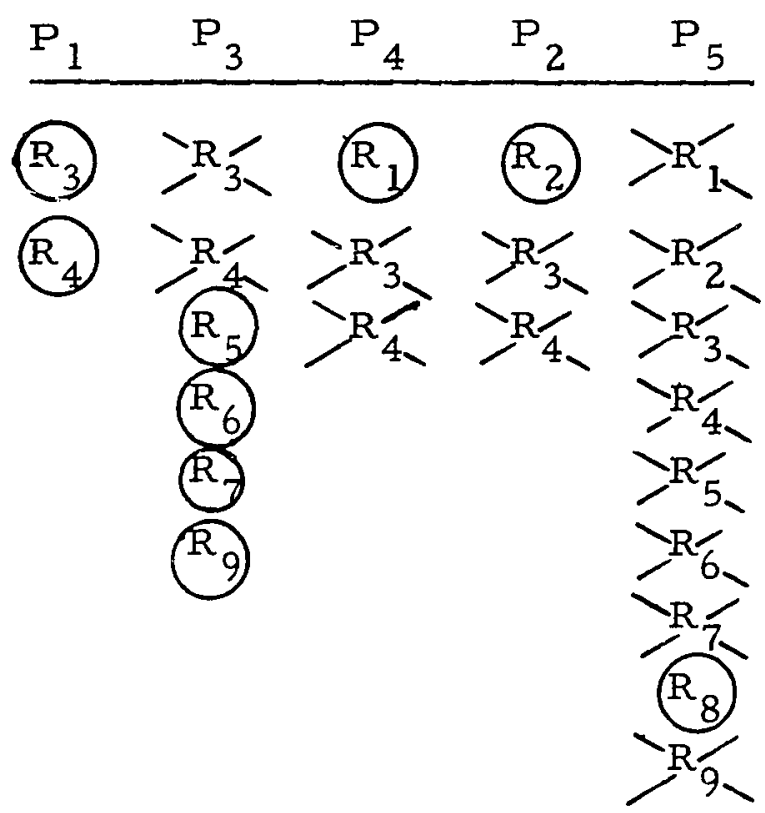


scale. If a resource variable appears under $P_{1}$, it is deleted from $\mathrm{P}_{2}$ $P_{5}$. Thus, $R_{3}$ and $R_{4}$ are classified as satisfying the requirements of $P_{1}$. This is indicated by circling $R_{3}, R_{4}$ under $P_{1}$, and deleting them from $\mathrm{P}_{2}-\mathrm{P}_{5}$. Continuing this procedure, each resource variable is classified under a single most suitable policy statement. The final results are:

$$
\begin{aligned}
& P_{1}=1, \text { for } R_{3}, R_{4} \text { (i.e., } R_{3}, R_{4} \text { meet the requirements of } \\
& \text { policy } \left.P_{1}\right) \\
& P_{3}=1 \text {, for } R_{5}, R_{6}, R_{7}, R_{9} \\
& P_{4}=1 \text {, for } R_{1} \\
& P_{2}=1 \text {, for } R_{2} \\
& P_{5}=1 \text {, for } R_{8}
\end{aligned}
$$

The implications of the above results for policymaking and planning purposes can now be discussed. The CT transfer is feasible in terms of $R_{3}$ (markets) and $R_{4}$ (tools, equipment). It is feasible in terms of $R_{5}$ (Quality of Labor), $R_{6}$ (Labor Force available for $C T$ ) and $R_{9}$ (Raw materials) if the constraints of low productivity of these resources can be overcome. The resource $R_{1}(R \& D$ base) is not available in India at the present time. To overcome this constraint and to make CT transfer feasible in terms of $R_{1}$, India should create proper training facilities and/or send trainees abroad. The later aspect is termed 'Antennae' by Spencer [88]. The transfer is feasible in terms of $R_{2}$ (capital) only if its high cost can be overcome or tolerated. This constraint may decelerate the transfer of CT to India. Finally, the transfer is infeasible 
in terms of $\mathrm{R}_{8}$ (managerial and entrepreneurial skills). This finding may suprise some Indian planners who do not attach high importance to the availability of managerial and entrepreneurial skills. It seems imperative that India direct substantial resources to produce better managers and entrepreneurs. It is also interesting to note that this analytical procedure points out the importance of entrepreneurship, since the previous empirical study and intuitive suggestions also rank the entrepreneur as a major vehicle for $\mathrm{T} T$.

In passing, it may be observed that the nature of the results of Table VI does not allow the use of relevance analysis to classify resource variables $R_{1}-R_{8}$ under the policies $P_{1}-P_{5}$. In the conventional relevance analysis $[10, p .219-223]$, the row entries are added and the various (resource) variables are ranked according to the magnitude of the resulting sums. It can be seen from Table VI that such a scheme would not work here.

\subsubsection{Applicability of Transfer Mechanisms (TT Efficiency)}

The $(T \times M)_{f}$ matrix specifies the mechanisms applicable for $C T$ transfer to India. The applicability analysis identifies the mechanisms (channels) most likely to produce the desired outcomes of TT.

The procedure outlined in section 3.10 .2 is used to determine the efficiency of TT in terms of the applicability of transfer mecha.nisms. For each element of the $(\mathrm{TxC})_{f}$ matrix having a value of 1 , we need to develop the three measures -- x,y, and $z$. Recall that the 
specification $x=1$ indicates that the mechanism has been used in past successfully at least once, $y=1$, implies the transfer is acceptable and $z=1$ if the mechanism is considered to be viable in the future. Specifications for $x, y, z$ for the applicable mechanism are based on the discussions in the following paragraphs and are shown in Table VIJ.

The military channel has been used extensively in the U.S. for transferring mainly hardware technology. As for India, its usefulness for CT transfer is low and will remain so [52, p.39]. Hence, $x, y, z=0$ for hardware. The assignment for $x, y, z$ for software and peripherals are unnecessary, since the first element of the second and third rows of the $(T \times M)_{f}$ matrix is zero.

The channel direct investment hy government has been used in the past to transfer all three CT attributes, e.g., in France. The Indian government makes large investments in modern technology. Hence $x=1$ for all three CT attributes. The government is a sluggish transfer mechanism, as demonstrated in the case of the steel $\mathrm{TT}$ undertaken by the Indian government $[18, p, 35]$. Hence, $y=1$ for all three CT attributes. The future applicability of this mechanism will remain high for India [18, Chapter 6]. Hence, $z^{-1}$ for the three CT attributes.

The direct investment by business nas been the most used mechaanism for the past CT transfers. The multinational corporations, such as IBM, have made direct investments in Western Europe [67,pp.78-88]. Hence, $x=1$ for all three attributes. Since the profits and return on investment are at stake, the time for transfer is usually reasonable. 
TABLE VII

SPECIFICATION OF APPLICABILITY MEASURES

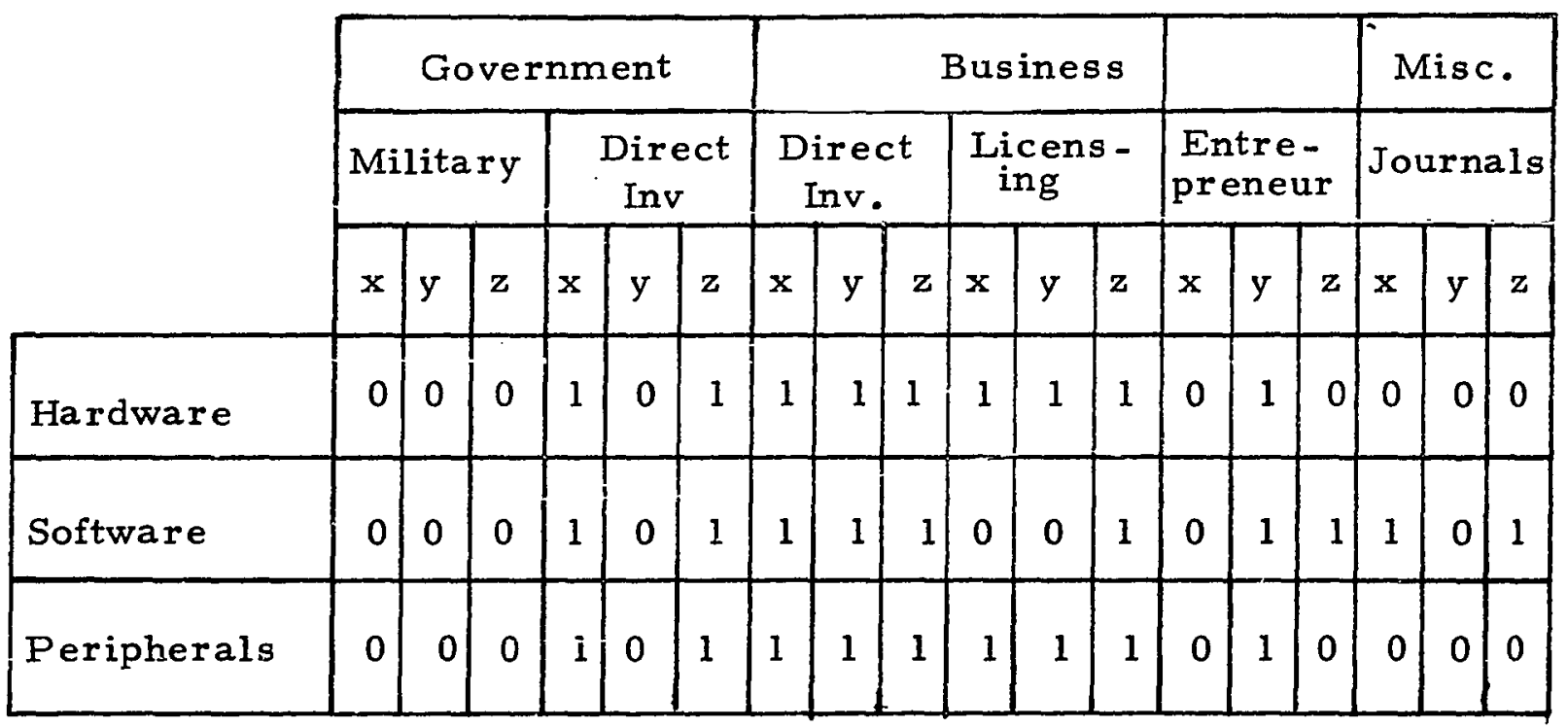


Since this is true for all three attributes, $y=1$, in all three rows. The multinational corporations are here to stay $[47, \mathrm{pp} \cdot 49-54]$. Hence, $z=1$ for the three CT attributes.

Licensing has also been used often to transfer CT. For instance, India has started assembling ICL computers under licensing. However, under most licensing schemes, only hardware and peripherals are developed; usually, software is the responsibility of the licensee [15]. Hence, $x=1$ for hardware and peripherais, but $x=0$ for software. The time for transfer through licensing channel is again a licensee's responsibility. The past studies (e.g., 67, pp.44-49) indicate that the time for transferring hardware and peripheral technology through the licensing channel has been reasonable, but software evolves slowly. Hence, $y=1$ for hardware and peripherals, and $y=0$ for software. The future applicability of the mechanism is expected to be high because a high percentage of the CT market is controlled by companies such as IBM, ICL, etc. It is most likely that company policies would improve the channel performance to transfer software also [15]. Heñce, $z=1$ is specified for all three CT attributes.

The entrepreneur seems to be a good mechanism to transfer software but not for hardware and peripherals on the large scale needed for India = Hence, we specify $x=1$ for software, and $x=0$ for haraware and peripherals. The time for transfer is usually reasonable because of the financial commitments made by the entrepreneur. Hence, $y=1$ for all three attributes. The future viability of this mechanism will 
likely prevail in software area. Hence, $z=1$ for software, and $z=0$ for hardware and peripherals.

The journals have been useful in the past to transfer software. They are sluggish but they will remain useful in the future. Hence, $x=1, y=0, z=1$ for software. The assignments of $x, y, z$ for hardware and peripherals are not necessary, as can be observed from the $(\mathrm{TxM})_{f}$ matrix.

Using the values of $x, y, z$ from. Table VII, policies $Q_{1}-Q_{5}$ are developed. It can be noted that the entries in Table VII for hardware and peripherals are identical. Thus, only two sets of $Q_{1}-Q_{5}$ are developed, one for software and another for hardware and peripherals. These are shown in Tables VIII and IX, respectively. Based on the discussion in section 3.10 .2 , the five policies $Q_{1}-Q_{5}$ can be ranked in the following order of preference: $Q_{1}, Q_{2}, Q_{4}, Q_{3}$ and $Q_{5}$. Since the technology is well-established in the U.S., $Q_{2}$ is preferred to $Q_{4} \cdot Q_{3}$ implies long transfer time; hence, it is less preferred than $Q_{1}, Q_{2}, Q_{4}$ but more than $Q_{5}$ Wich is an unrealistic policy statement. $Q_{1}$ is the most preferred one, since it implies $x, y, z=1$.

From Table VIII, for software, the results are:

$$
\begin{aligned}
& Q_{1}=1 \text { for } M_{3} \\
& Q_{2}=1 \text { for } M_{3} \\
& Q_{4}=1 \text { for } M_{3}, M_{5} \\
& Q_{3}-1 \text { for } M_{2}, M_{3}, M_{6} \\
& Q_{5}=1 \text { for } M_{2}-M_{6}
\end{aligned}
$$


TABLE VIII

POLICY STATEMENTS FOR APPLICABILITY OF

TRANSFER MECHANISM: SOFTWARE

\begin{tabular}{|c|c|c|c|c|c|c|}
\hline & & \multicolumn{5}{|c|}{ Policy Statements } \\
\hline & & $Q_{1}$ & $\mathrm{Q}_{2}$ & $Q_{3}$ & $\mathrm{Q}_{4}$ & $Q_{5}$ \\
\hline \multirow{2}{*}{$\begin{array}{l}\stackrel{+}{3} \\
0 \\
ن\end{array}$} & Military $-M_{1}$ & 0 & 0 & 0 & 0 & 0 \\
\hline & Dir. Inv. $-\mathrm{M}_{2}$ & 0 & 0 & 1 & 0 & 1 \\
\hline \multirow{3}{*}{ 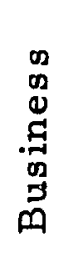 } & Dir. Inv. $-M_{3}$ & 1 & 1 & 1 & 1 & 1 \\
\hline & Licensing $-\mathrm{M}_{4}$ & 0 & 0 & 0 & 0 & 1 \\
\hline & Entrepreneur $-M_{5}$ & 0 & 0 & 0 & 1 & 1 \\
\hline 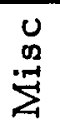 & Journals $-M_{6}$ & 0 & 0 & 1 & 0 & 1 \\
\hline
\end{tabular}


TABLE IX

POLICY STATEMENTS FOR APPLICABILITY OF

TRANSFER MECHANISMS: HARDWARE

AND PERIPHERALS

\begin{tabular}{|c|c|c|c|c|c|c|}
\hline & & \multicolumn{5}{|c|}{ Policy Statements } \\
\hline & & $Q_{1}$ & $Q_{2}$ & $Q_{3}$ & $\mathrm{Q}_{4}$ & $Q_{5}$ \\
\hline \multirow{2}{*}{$\begin{array}{l}\frac{1}{Z} \\
0 \\
\mathcal{Z}\end{array}$} & Military $-M_{1}$ & 0 & 0 & 0 & 0 & 0 \\
\hline & Direct Inv. $-M_{2}$ & 0 & 0 & 1 & 0 & 0 \\
\hline \multirow{3}{*}{ 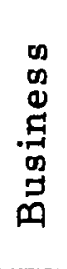 } & Direct Inv. $-\mathrm{M}_{3}$ & 1 & 1 & 1 & 1 & 1 \\
\hline & Licensing $-\mathrm{M}_{4}$ & 1 & 1 & 1 & 1 & 1 \\
\hline & Entrepreneur $-M_{5}$ & 0 & 0 & 0 & 0 & 0 \\
\hline 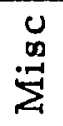 & Journals $-M_{6}$ & 0 & 0 & 0 & 0 & 0 \\
\hline
\end{tabular}


It is clear that $M_{1}-$ - military channel -- is not useful for CT transfer to India. Using the procedure described in previous sections to classify a mechanism under a single policy statement, the final results are:

$$
\begin{aligned}
& Q_{1}=1 \text { for } M_{3} \\
& Q_{4}=1 \text { for } M_{5} \\
& Q_{3}=1 \text { for } M_{2}, M_{6} \\
& Q_{5}=1 \text { for } M_{4}
\end{aligned}
$$

Since $Q_{3}$ is least preferred and $Q_{5}$ is unrealistic, the applicable mecha nisms for software $\mathrm{TT}$ to India are $\mathrm{M}_{3}$ (Direct Investment by Business) and $M_{5}$ (Entrepreneur). The mechanismas $M_{2}$ (Direct Investment by Indian Government) and $M_{6}$ (Journals) are applicable if sluggish transfer can be tolerated.

For hardware and peripherals, Table IX yields:

$$
\begin{aligned}
& Q_{1}=1 \text { for } M_{3}, M_{4} \\
& Q_{2}=1 \text { for } M_{3}, M_{4} \\
& Q_{4}=1 \text { for } M_{3}, M_{4} \\
& Q_{3}=1 \text { for } M_{2}, M_{3}, M_{4} \\
& Q_{5}=1 \text { for } M_{2}, M_{3}, M_{4}
\end{aligned}
$$

The transfer mechanisms $M_{1}, M_{5}, M_{6}$ are not applicable for this case. The mechanisms $M_{3}$ (Direct Investment by $\mathbf{L}:$ siness) and $M_{4}$ (Licensing) are most preferable to transfer hardware and peripheral technology to India. The channel $\mathrm{M}_{2}$ (Direct Investment by Indian Government) is appiicable, if long transfer time is acceptable. 
Additior 1 policymaking and planning implications resulting from the above analysis will be discussed in section 4.7 .

\subsubsection{Desirability of the Consequences of CT Transfer to India}

The technique outlined in section 3.10 .3 can be applied to determine the desirability of the consequences of CT transfer to India.

The $(\mathrm{TxC})_{\hat{I}}$ matrix is partitioned into two submatrices: $(\mathrm{TxC})_{\hat{f 1}}$ contains zero elements and $(\mathrm{TxC})_{\mathrm{f} 2}$ contains unity elements of the $(\mathrm{TxC})_{f}$ matrix. These submatrices are shown in Figures $33(\mathrm{a})$ and $34(\mathrm{a})$. The zero entry in $(T \times C)_{f}$ and $(T \times C)_{f l}$ matrices indicates a lack of interaction between the corresponding two variables. We need to determine if the lack of interaction creates a misfit, as discussed in section 3.10 .3 . If a misfit occurs it is indicated by a 1 in the matrix $(\mathrm{TxC})_{\mathrm{ml}}$ shown in Figure $33(\mathrm{~b}) . \quad(\mathrm{TxC})_{\mathrm{ml}}$ is the matrix of misfit variables created by a lack of interaction. Similarly the matrix $(\mathrm{TxC})_{\mathrm{m} 2}$ is developed in Figure $34(\mathrm{~b})$ for the misfit variables created by the existence of an inter action.

The $1^{\prime} \mathrm{s}$ in $(\mathrm{TxC})_{\mathrm{ml}}$ matrix in Figure $33(\mathrm{~b})$ were specified by considering that the lack of impact of hardware and peripheral technology on the variables quality of medical care, extent of mass communication, and food/capita creates a misfit, since these variables seem to present challenging problems to India's development. Howrever, the lack of impact of these two CT attributes on the variable-character of basic social organizations -- is considered not to create a misfit because it 


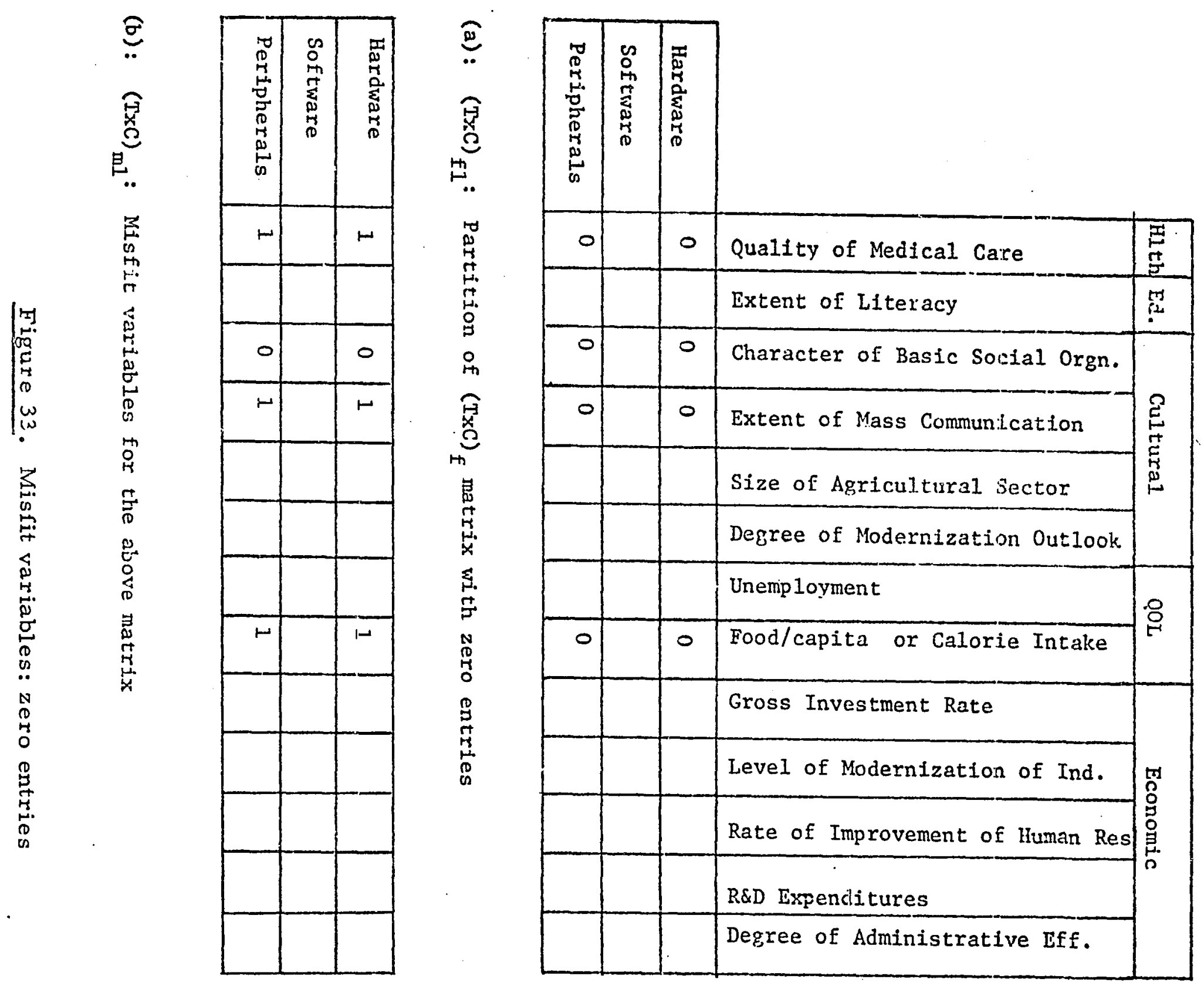



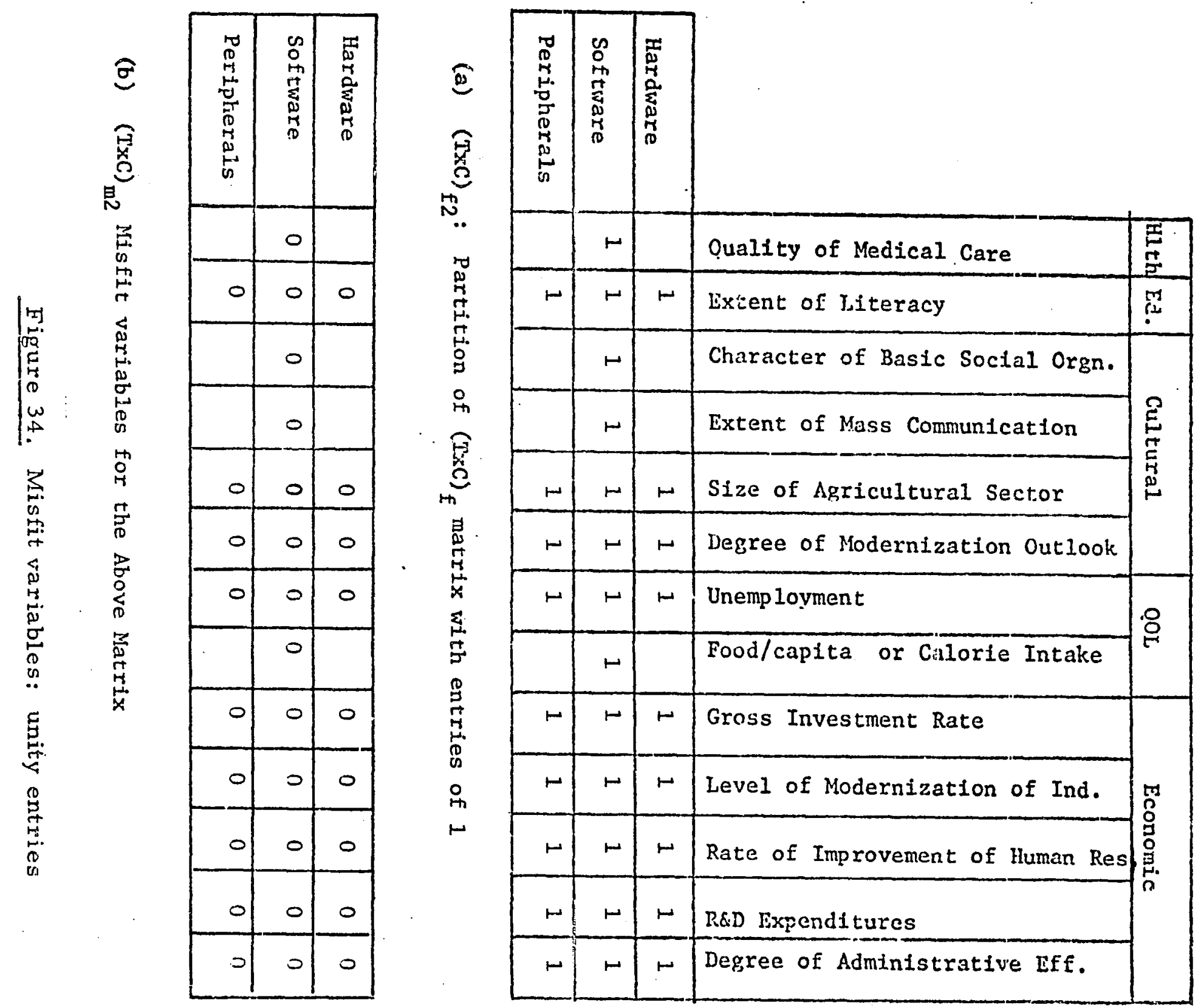
seems desirable to maintain the present character of basic social organization consisting of closely-knit family units. This is a subjective judgment, supported by discussions in references 18, pp.25-32, and 75, pp. $283-290$

Since an entry of 1 in the $\left(T_{x} C\right)_{f}$ matrix signifies desirable impact, it is easy to sperify that mis fit does not occur for all entries of (TxC) $\mathrm{m}^{\text {* }}$ Therefore, ail elements of this matrix are zero.

The two submatrices $(\mathrm{TxC})_{\mathrm{m} 1}$ and $\left(\mathrm{TxC}_{\mathrm{m} 2}\right.$ are synthesized to generate the $(\mathrm{T} \times \mathrm{C})_{\mathrm{m}}$ matrix of Figure 35 . The $\mathrm{l}^{\prime} \mathrm{s}$ in this matrix indicate presence of a misfit between the corresponding two variables.

From Figure 35, it can be concluded that software produces no misfit and hence, it is the most desirable CT attribute. Both hardware and peripheral technology create misfit in terms of the following variables: quality of medical care, extent of mass communication and food/ capita. The implication here is that India should concentrate and be concerned more about software technology than hardware and peripheral technologies. Of course, the latter two technologies are necessary integral parts of the total CT, but they should be treated as means rather than ends.

Additional implications of the above analysis are considered in the following section.

\subsection{Leval 3 Requirements}

The Levels 1 and 2 were developed within the larger framework supplied by Level 3. It can be observed that all logic, decision and 


\begin{tabular}{|c|c|c|c|c|}
\hline 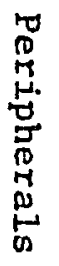 & 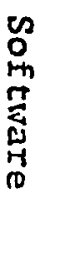 & 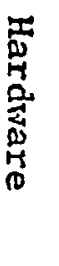 & & \\
\hline$\omega$ & 0 & $\leftarrow$ & Quality of Medical Care & 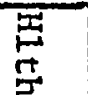 \\
\hline 0 & 0 & 0 & Extent of Literacy & M \\
\hline 0 & 0 & ○ & Character of Basic Social Orgn. & \multirow{4}{*}{ 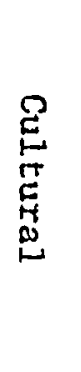 } \\
\hline$\omega$ & ○ & 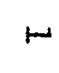 & Extent of Mass Communication & \\
\hline o & ○ & 0 & Size of Agricultural Sector & \\
\hline 0 & 0 & 0 & Degree of Modernization Outlnok & \\
\hline 0 & 0 & 0 & Unemp loyinent & \multirow{2}{*}{8} \\
\hline$r$ & 0 & $\mapsto$ & Food/capita or Calorie Intake & \\
\hline 0 & 0 & 0 & Gross Investment Rate & \\
\hline 0 & 0 & 0 & Level of Modernization of Ind. & \\
\hline 0 & 0 & 0 & Rate of Improvament of Human Res & \\
\hline 0 & 0 & 0 & R\&D Expenditures & \\
\hline 0 & 0 & 0 & Degree of Adininistrative Eff. & \\
\hline
\end{tabular}


information requirements specified by the Level 3 flowgraph (Figure 14) have been considered.

\subsection{Implications for Policymaking and Planning}

In this section, the results of previous sections and the role of CT transfer are examined in terms of planning and policymaking aspects of TT. Before this is done, a framework for discussing planning and policymaking issues will be established.

It seems useful to consider planning as a hierarchy of three levels: policy, strategic and operational planning [44, p.15]. At the policy planning level, concern is to establish new norms and setting of alternate goals. At the strategic level, alternate means to attain the set goals are of interest. At the operational planning level, the implementation of selected strategy is of importance. The policymaking process is best illustrated by quoting Vickers [100,p.116]:

"Policy (consists) in regulating a system over time in such a way as to optimize the realization of many conflicting relations without wrecking the syster. in the process".

In the present discussion, emphasis will be placed on policy and strategic planning rather than operational planning. Hence, the usefulness of the resuits of previous sections is discussed in terms of these two dimensions of planning.

For TT, the needs of policy and strategic planning and policymaking can be translated into the following, specific generating questions: 
Question 1: What is the best form of CT transfer to India? Is it better to borrow it from "bottom-up" or parts of it?

This question is related more to policy planning because it helps establish goals for CT transfer to India. Furthermore, it opens up avenues for considering alternate goals. Question 2: Which transfer mechanisms are useful?

Question 3: What are the resource requirements?

Question 4: What are the consequences of $\mathrm{TT}$ ?

These questions are related partly to policy planning and partly to strategic planning. In terms of policy planning, the answers to these questions can help assess the realizability of set goals, and hence, they provide a way to select among alternate goals.

In terms of strategic planning, the answers help us in designing alternate strategies for achieving the stated goals. The question concerning mechanisms helps identify the alternate ways to transfer CT to India. The answers to the question dealing with resource requirements aids (strategic) planning of resources. The anticipations in terms of the consequences of CT can supply selection criteria for choosing among the available strategies.

Question 5: Is the CT transfer to India feasible in terms of required resources?

Question 6: Is the transfer efficient?

Question 7: Is the transfer desirable in terms of the consequences of CT? 
These questions are related to strategic planning mainly because they help evaluate the strategies available to the designer. Such answers can also be useful in policy planning. These answers, therefore, provide feedback from strategic to policy planning indicated in Figure 2 .

Question 8: Does the CT transfer to India improve the system, considering India as a system?

This question can at best be answered in a qualitative way, mainly by considering the consequences of CT transfer to India. Here, one can: (a) identify the consequences that are desirable in terms of system improvement, and (b) those that are bottlenecks to system improvement.

Question 9: How does the proposed IS help the policymakers and planners? If the questions 1-8 can be answered in a useful manner, one can propose that these clients of IS have been properly served.

The usefulness of the suggested approach can be illustrated by determining how well the eight questions can be answered, using the results obtained in previous sections.

Answer 1: The Best form of CT Transfer to India.

The results of previous sections indicate that the best form of CT transfer to India is software. The elements of the $(\mathrm{TxC})_{f}$ matrix (Figure 32) and the analysis for desirability of TT indicate that software creates no misfit and hence, is the best form (of CT) to meet demands of the context. Of course, it is obvious that one cannot have software 
without proper hardware or peripherals. However, it seems important to realize that without proper software (especially application), little can be gained in terms of sociocultural improvement.

It also seems useful to extend the implications of above comments to manpower requirements. On the basis of this analysis, the need for programmers, software specialists and entrepreneur skills seems more important than the need for manpower to build hardware and peripherals, since the latter can be assembled under licensing agreements.

The suggestion for policy planning, as far as the best form of $\mathrm{CT}$ is concerned, is to emphasize software as the principal and hardware and peripheral as the complimentary technologies. Answer 2: Us eful Mechanisms for CT Transfer to India

The $\left(T_{x M}\right)$ matrix (Figure 31$)$ specifies the mechanisms likely to be useful for CT transfer to India. The analysis for TT efficiency or applicability of transfer mechanisms indicates that direct investment by business and licensing are the most useful channels for transferring hardware and peripherals, while the former channel seems more efficient for software. If a sluggish transfer can be tolerated, direct investment by the Indian government is also an efficient mechanism.

In terms of policy planning, the impiication is that the goals set by the Indian government (e.g., in the five-year plans) should allow for facilitating and encouraging business mechanisms. The government should seek licensing arrangements with the leading computer companies. 
The goals set must also allow for speed-up of the transfer, if the government wishes to act as the transfer mechanism. This is important, since large investments in India are made in the public sector. For strategic planning, the $\left(\mathrm{T} \times \mathrm{M}_{\mathrm{f}}\right.$ matrix identifies alternate mechanisms available for CT transfer to India. The Indian planners and policymakers can eithe $r$ utilize the channels that seem efficient according to this analysis, or they should attempt to overcome the constraints of other channels to make them equally efficient. Answer 3: Required Resources for CT Transfer to India

The $(T \times R)_{f}$ matrix (Figure 30$)$ indicates resources required for CT transfer to India. Although the information is limited to whether a resource is required or not, it is useful for both policy and strategic planning, since only the needed resources can then be examined in detail.

In terms of policy planning, the information concerning the resource requirements can be useful in assessing the realizability of the goals set by the government or a planning commission. The identification of the requirements and constraints on the resources eases the task of making such an assessment. Also, the ( $T x R)_{f}$ matrix provides a means to test the feasibility of $\mathrm{TT}$ in terms of the resources required.

The planning of resources, or resource allocation, is important in strategic planning efforts. Again, the $(T \times R)$ matrix provides useful inputs to resource allocation process by specifying which 
resources are needed for CT transfer to India. Furthermore, the $(T \times R)_{f}$ matrix contains information about the impact of one resource requirement on another. The result of considering such self-interactions among various resources is that the $(T \times R)_{f}$ matrix shows the need and importance of all resource variables specified in Level 1 . If self-interactions were ignored, resource planners, would have ended up with an incomplete list of resource variabies.

\section{Answer 4: Consequences of CT Transfer to India}

The $\left(\mathrm{TxC}_{\mathrm{f}}\right.$ matrix indicates the impact or consequences of $\mathrm{CT}$ on sociocultural and economic environment of India. It can be observed from the matrix that software impacts most. Also, on the whole, the economic variables are significantly impacted by the three CT attributes but the sociocultural variables are not. Therefore, the nature of the $(\Gamma \times C)_{f}$ matrix indicates the so-called resilience of the sociocultural variables.

In terms of policy planning, the information concerning the consequences can again help establish proper goals for social futures of India. It has been observed by some (e.g. reference 2, Chapter VIII) that a change in the basic social structure and outlook for modernization is essential to rapid economic growth. However, the $\left(\mathrm{TxC}_{\mathrm{f}}\right.$ matrix indicates that the transfer of CT does not seem to significantly affect such variables. Thus, it seems that only computerization on a massive scale can change the nature of such variables; that is, the utilization of computer rather than manufacture can bring the effects 
of CT sufficiently close to the large masses of India so as to generate a change in their outlook.

Strategic planners and policymakers can utilize the information about the impacted consequences by emphasizing those strategies capable of eliminating misfits created in terms of various sociocuitural variables, e.g., the quality of medical care. Since the impact on economic variables is found significant, attention should be given to the strategies that can accelerate economic changes by accelerating sociocultural changes. Answer 5: Feasibility of CT Transfer to India

The analysis for feasibility in terms of the requirements of various policy statements indicates that the CT transfer is feasible in terms of market availability and tools and equipment needed for CT. The feasibility in terms of other resources is possible only under some constraints, with the exception of the resource -- managerial and entrepreneurial skills -. which is found infeasible.

The feasibility analysis suggests that CT transfer to India faces the constraint of high costs of capital. The computer industry is both capital and labor intensive; in fact, it appears to be more capital intensive than labor intensive. Therefore, the Indian government must plan for either paying the high cost of capital (if it is available from abroad), or absorb locally some of the capital requirements through an early start on CT development. The planning for capital is important, 
since the Indian government has consistently underestimated the foreign aid and exchange requirements. For instance, Lewis states [52, p. 24]:

"The plan (Fourth five year plan) otherwise is a cogent, pragmatic, agriculture-accenting document; it is more realistic than its predecessor five-year plans..., except that it seriously under states the aid requirements. Its five-year calculation of needed net aid is short, I suspect, by something like a billicil dollars."(!)

It seems then the generation of capital is of the utmost importance. (Lewis suggests many ways to do this -. in particular, he recommends revising the fiscal and savings policies of the Indian Government.) The other critical resource, found infeasible, is the lack of managerial and entrepreneurial skills. Some comments can be made in this respect. India has a large number of people who have gained little from the development that has taken place so far. There is a vast amount of untapped human skills, especially in the rural areas. It appears that the needed skills are likely to come from these areas. Hence, the policy should be designed to provide a better education in the areas -- better in terms of staffing, programs and general effectiveness. Also, it seems that primary rather than secondary or higher educcation should be emphasized because it is estimated that about onethird of the pupils drop out during the first six years of elementary schools.

Other resources, feasible, but under constraints, are: $R \& D$ base, size and productivity of the labor force for CT, technical and scientific skilis and raw materials. The present trends indicate that 
these constraints are not serious, and they can be overcome by the programs started by the state and central governments of India. For instance, the $R \& D$ base is already growing in India at the rate of $2.3 \%$ per year [18,pp.306-308]. The occupational and vocational training programs started by the Indian government can be expected to counter the productivity constraint on the human resources. Answer 6: CT Transfer Efficiency

The analysis in section 4.6.2 indicates that direct investment by business is the most efficient channel, since it can be utilized to transfer all three CT attributes. Other efficient channels are: licensing for hardware and peripheral equipment, and entrepreneur for software. The channel .. direct investment by the Indian government .. is less efficient, if the time for transfer is considered an important parameter. Other channels are found inapplicable.

The implications of using business channels are interesting. Traditionally, large investments (e.g., steel, fertilizer) in India have been allowed only in the public sector. Moreover, most industries in the public sectors are prote -ad by the government, thus stifling the competitive spirit of a free market economy. For instance, as soon as a product is made in India, import restrictions are put on the product, thus minimizing competition and providing protection to domestic manufaturers. Such policies have discouraged foreign companies from investing in India. In spite of the emphasis on this issue by many economists $[18,77,42,52]$, the Indian government has failed to 
establish policies suitable for stimulating large-scale investment by businesses -- either domestic or abroad. It appears that such an investment would allow market competition resulting in better goods and services and investment opportunities [52,pp.35-37; 77,pp.232 235].

The above issue, therefore, presents a dilemma to the Indian policymaker. Oñ oñe hând, the business channeis seem efficient to transfer CT. But, on the other hand, the government discourages heavy investments by business. Although suggesting a proper solution to this dilemma requires a study on its own merits, the implication for CT transfer to India are as follows. What is needed is balanced policies that can regulate investments in the following way: use the government as a TT mechanism in cases where sluggish transfer can be tolerated, and employ business channels where time to transfer is of major concern. Thus, for CT: use the government mechanism to transfer hardware and peripheral technologies under licensing from foreign companies, and develop software through business channels. It seems wise to transfer the three components of $\mathrm{CT}$ through these different mechanisms. Answer 7: Desirability of Consequences of CT

The analysis in section 4.6 .3 indicates that $C T$ is desirable in terms of all economic variables. The impact of $C T$, however, is not uniform on sociocultural variables. 
It seems most desirable to emphasize software development. As suggested earliex, hardware and peripherals, although necessary, do not provide the desirable impact on sociocultural variables. However, the impact of technology on the social fabric of a country is es sential in its economic development [2, Chapter VIII]. For instance, Rostow points out $[78, p .174]$ :

"I take growth to be one manifestation of much wider process of modernization forced upon more backward nations by the consequences of failing to modernize in our inherently competitive and continuous arena of world power".

Therefore. misfits created in sociocultural variables should not be considered distinct from or independent of the economic variables and vice versa.

In terms of strategic planning, the analysis for the desirability of consequences points out the essential fact that the sociocultural variables are strategically as important as economic variables. Hence, strategies should be selected to minimize or eliminate misfit between the CT attributes and the corresponding sociocultural variables, in particular - - the quality of medical care, the extent of mass communication and food/capita, since these variables are characteristic of some of the pressing problems faced by India.

Food/capita or the calorie intake of an Indian is one of the lowest among all nations of the world. Therefore, it is important to consider how CT can improve this sector. In particular, the impact of 
software on agricultural yields should be investigated (e.g., references 65, pp.188-199 and 5 present some ideas in this respect).

The sociocultural variable -- extent of mass communication -is important, since a lack of proper communication inhibits the use of research and advanced farming techniques by the Indian industries and farmers. In this respect, diffusing the existing knowledge to the rural parts of India is an important task faced by the Indian planners and policymakers.

Correa [23, pp.30-38] reports that labor productivity can be considerably increased by proper nutritional and medical care. The analysis for resource feasibility indicated the need to improve the labor productivity. Therefore, it seems imperative to design policies to eliminate misfit between the CT attributes and tha variable -quality of medical care.

Answer 8: System Improvement

Here, we extend the implications of the results obtained to assess the ability of CT to contribute to system improvement, considering India as a system. We also attempt to consider in this section how the environment, e.g. political climate, can affect the decisions to transfer CT.

In order to answer this, consider the challenging problems faced by the system. First, the size of population which is growing at an alarming rate. Second, one effect of technology has been the creation of worldwide uniformity of individual needs and desires. This leads 
to the rise of expectations of Indian people; most of these expectations remain unfulfilled. Third, a multiplicity of languages and difficulties of communication prevents the distribution of relevant information and ideas, which can bring about and sustain the changes. Fourth, and this may be quite important, the accumulation of knowledge has reached enormous proportions and is accelerating so rapidly that keeping abreast of scientific and technical developments is highly difficult.

Let us then consider how and to what extent the CT transfer can contribute to system improvement in these four dimensions.

First, the popuiation explosion. The increasing population puts strong and heavy demands on India's resources, especially the food production. The ability of CT to improve the food production was mentioned in the previous section. The hope here is the increase in the number of models and simulations being developed to help agricultural planning. Such efforts can produce significant improvement in agricultural outputs [65, pp.169-182 and 189-203]. The CT can also help, in the fiture, act of better weather forecasting. The improvements in weather forecasting can lead to obvious but significant improvements in agricultural production.

Secondly, the expectations of an average Indian. The CT transfer alone cannot increase the possibility of meeting expectations of Indians. However, the analysis on the desirability indicates that significant improvement in the economic sector can result from the CT traisfer. For example, consider the impact of CT on just the administrative 
efficiency. The efficiency of Indian bureaucracy is poor [e.g., 18, pp.24-25]. Therefore, if an area of overwhelming public interest is considered for computerization, it would be administrative tasks.

Another expectation of an average Indian is job security. In this respect, it has been suggested by some that computerization may create unemployment. That such a suggestion is a myth can best be illustrated by the following quote from Butani $[18, p, 121]$ :

"So far as labor is concerned, computerization would mean, in the aggregate, a far larger volume of employment than a noncomputerized society can ever offer. The very nature of computerization and the extremely large number of elements that go into the system from manufacturing to operation, would open out whole new fields of employment for our young vo:?s and girls".

Third, the aspects of communication. It seems worthwhile to consider an analogy here. The computer is a vital and useful element of the communication links existing in the Western world and Japan. Therefore, the potential of $\mathrm{CT}$ in improving the efficiency of communication links can be significant for India also.

Fourthly, the diffusion of knowledge. Here both the management and communication of knowledge seem important. The enhancernent of communication capability through the use of computers has been demonstrated in many sectors of the world. As far as the management aspects are considered, again Butani is quoted $[18, \mathrm{p} .121]$ :

\footnotetext{
"... computerization is likely to have an upgrading influence on the quality of management, so far developed in the country. Instead of the
} 
order-shouting, decision-by-hunch, management-by-bluff type that we have, computerization would compel the emergence of a highly professionalized management, which understands what mathematical parameters are, and has the capacity to react intelligently..."

Hence, proper utilization of CT can help diffuse the knowledge, especially in the rural India.

It also seems useful to consider the effect of some environmental parameters on $C T$. It appears that economic environment is ripe to realize the benefits of CT transfer to India. In twenty-five years, the Indian industrial base has grown substantially: for instance, the production of crude oil rose from 26,000 in 1947 to nearly 7 million tons in 1969 , the production of electricity from 4 billion $\mathrm{kwH}$ to 50 billion kwH, steel production rose from 893,000 to nearly 6 million tons. India.'s real GNP is expanding at a rate of $3-1 / 2 \%$, higher than U.S. Althcugh the overwhelming population dwarfs the results of such progress, the growth and expansion of this nature seem conducive to CT transfer.

At the professional level, India has built a techno-structure of enormous magnitude. The need to improve the efficiency of this technostructure can also be satisfied through proper utilization of computers.

The political system in India has remained quite resilient so that it seems unlikely that the Indian Union will break up. In fact, India is an example of a workable common market concept. The 
military has maintained self restraint for the last 25 years. As Lewis [52, pp.31-39] remarks, the self-restraint by the military confuses him, for he cannot find a good explanation for it. The Indian government is also realizing that Ludia is too large a system to govern and therefore, is attempting to generate better means to control and monitor the system performance. In this respect, the impact of the political environment on CT (and vice-versa) can be significant. Answer 9: If the Clients of IS are properly served

The above discussion provides answers to the important generating questions outlined in section 1.3. They also aid the clients of the IS in making better decisions, especially for policy and strategic purposes; in this respect the IS alerts the clients by pointing out the likely to be the bottlenecks. One also hopes that the Singerian characteristics of this inquiry would be helpful in the planning and policymaking aspect of $\mathrm{TT}$.

One final reflection. The above discussion in essence indicates the desirability of $\mathrm{CT}$ and need for proper planning and policymaking which can render such a transfer feasible in terms of the required rescurces, efficient in terms of transfer mechanisms and desirable in terms of consequences. That such efforts are imperative can be illustrated by considering that India is at the "take-off" stage, in terms of the stages of growth proposed by Rostow [78, Chapter 2]. Once "take-off" occurs, growth is self-sustained for some time to come. 
In this respect, the following quote from Rowtow seems especially useful and relevant in terms of preconditions of take-off $[78, \mathrm{p} .6]$ :

"The more general case in modern history, however, saw the stage of preconditions arise not endogeneously but from some external intrusion by more advanced societies. These invasions -literal or figurative -- shocked the traditional society and began or hastened its undoing; but, they also set in motion ideas and sentiments which initiated the process by which a modern alternative to the traditiona.l society was constructed out of the old culture". 


\section{CHAPTER V}

\section{CONCLUSIONS, EXTENSIONS}

\subsection{Conclusions}

5.1.1 With a growing need to transfer technology as one of the means to reduce the technology gap between developed and developing regions, it seems important to understand the structure of the problem of TT ('Technology Transfer). In particular, answer to generating questions related to structure, feasibility, efficiency and desirability of TT need to be answered

5.1.2 Most of the previous inquiries into $T T$ represent unidimensional points of view on $\mathrm{TT}$, such as human factors, entrepreneurship, economic and informational aspects. Despite the bulk of such work, lack of an integrative (systems) approach to TT is evident. Two analytical studies by Spencer and Woroniak $[88,90]$ attempt to quantify $\mathrm{TT}$, mainly in terms of economic variables. Another analytical approach, by Kementa [47], has been found to have little practical application. The network model pruposed by Bar-Zackāy [11] is probabiy the most comprehensive one, but it is only descriptive and does not specify explicitly the structure ô̂ the problem; 
consequently, its usefulness in terms of prescriptions for planning and policymaking is limited. Doctors' empirical studies point out a need for a better approach to understanding $\mathrm{TT}$.

The major contribution of previous studies on TT has been their highlighting of the various aspecis of $\mathrm{TT}$ and their providing ar under standing of some basic issues.

5.1.3 It seems worthwhile to compare these previous approaches with the methodology described in this thesis.

Recall that in Chapter II, the previous approaches wers grouped into three broad categories:

1. Descriptive: These approaches attempt to explain the TT process verbally, and the verbal models often remain unstated and implicit. The three models, described in reference 35 (pp.4-8) and reproduced in Figures $3(\mathrm{a}),(\mathrm{b})$ and (c), and Spencer's work (particularly reference 89 ), are typical examples of these approaches.

2. Analytical and Empirical: The analytical approaches try to be quantitative and often employ mathematical relationships to describe the various aspects of the $\mathrm{TT}$ process; for instance, Minasian's [63] and Kmenta's [47] work. The empirical approaches try to explain the TT process on the basis of available data or by systematic collection of data, such as questionnaires. The premise here is that these data contain implicitly the explanation of the phenomenon underlying the TT process. 
3. Network: This approach specifies how various aspects, activities or stages of the TT process can be related in a network fashion. Often, the underlying principle is the one behind PERT (Program Evaluation and $\underline{\text { Review }}$ Technique). The only network model developed to examine the TT problem is the one by Bar-Zakay [11]. Table $X$, on the following page, is a summary comparison of the methodology proposed here with the three previous approaches.

It should be roted that the comparison is made on the basis isi twelve criteria. Thege criteria consider if the approach:

1. examines explicitly the planning and policymaking aspects of TT.

2. employs a multidisciplinary structure of $\mathrm{TT}$.

3. can be operationalized.

4. allows the decisionmaker to translate his mental models and subjective judgment.

5. can readily use the empirical data, if available.

6. requires computer facilities.

7. allows the inclusion of the group response.

8. needs modest or large scope of efforts to operationalize.

9. provides explicit measures to assess the $\mathrm{TT}$ performance.

10. is such that its outputs can be readily verified.

11. deals with the phenomena of discounting and resistance to long-range planning. 


\begin{tabular}{|c|c|c|c|c|c|}
\hline & Method (Technique) & $\begin{array}{c}\text { Proposed } \\
\text { Methodology }\end{array}$ & $\begin{array}{l}\text { Descriptive } \\
\text { Approaches }\end{array}$ & $\begin{array}{l}\text { Analytical } \\
\text { Empirical } \\
\text { Approaches }\end{array}$ & $\begin{array}{l}\text { Network } \\
\text { Approach }\end{array}$ \\
\hline 1 & $\begin{array}{l}\text { Explicit consideration of planning and policy- } \\
\text { making aspects of TT; Provides prescription } \\
\text { io planners and policymakers. }\end{array}$ & Yes & $\begin{array}{l}\text { Can be } \\
\text { extended }\end{array}$ & No & $\begin{array}{l}\text { Can be } \\
\text { extended }\end{array}$ \\
\hline 2 & Multidisciplinary structure of TT employed & Yes & Yes $^{1}$ & No & Partially \\
\hline 3 & The method can be operationalized & Yes & No & Yes & Yes ${ }^{3}$ \\
\hline 4 & $\begin{array}{l}\text { Allows decisionmaker to translate his mental } \\
\text { model and subjective judgment in the analysis }\end{array}$ & Yes & Yes & No & Partially \\
\hline 5 & $\begin{array}{l}\text { Empirical data, if available, can be used } \\
\text { readily in raw form }\end{array}$ & No & No & Yes & No \\
\hline 6 & Requires computer facilities & No & No & Yes & No \\
\hline 7 & Group response cañ be included & Yes & Yes & No & Yes \\
\hline 8 & Scope of efforts needed to use the method & Modest ${ }^{3}$ & Large ${ }^{2}$ & Modest: & $\begin{array}{l}\text { Modest - } \\
\text { large }\end{array}$ \\
\hline 9 & $\begin{array}{l}\text { Explicit measures provided to assess the per- } \\
\text { formance of } \mathrm{TT} \text {. }\end{array}$ & Yes & No & No & No \\
\hline 10 & $\begin{array}{l}\text { Results or outputs of the method can be readily } \\
\text { verified by the user. }\end{array}$ & $\mathrm{No}^{4}$ & $\mathrm{No}^{4}$ & $\mathrm{No}^{4}$ & No ${ }^{4}$ \\
\hline 11 & $\begin{array}{l}\text { The phenomena of discounting and resistance to } \\
\text { long-range planning can be considered in the } \\
\text { method }\end{array}$ & Yes ${ }^{1}$ & $\mathrm{Yes}^{1}$ & No & No \\
\hline 12 & $\begin{array}{l}\text { The method allows adaptation of environmental } \\
\text { changes in the analysis }\end{array}$ & Yes ${ }^{1}$ & Yes 1 & $\mathrm{No}^{2}$ & No \\
\hline
\end{tabular}

1. Method/Technique needs nodification/extension to satisfy this criterion

2. Method/Technique can be used but with increased difficulty in operationalizing it.

3. Modest, considering that the method utilizes a multidisciplinary representation of TT.

4. All four approaches require that the user specify monitoring signals to test the 'goodness' of the outputs provided by the method. 
12. allows adaptation of environmental changes.

These criteria, except numbers 6 and 8 , were selected mainly in terms of the implications of the Generating Questions of section 1.3, and the discussion, in section 1.4 , on planning and policymaking aspects of TT. Criteria 6 and 8 were selected because of their relevance to the purposes of this comparison and to user requirements in general.

We can draw these major conclusions from Table $X$ :

1. The method proposed here is better in terms of the igsues related to the TT structure, planning and policymaking aspects of $\mathrm{TT}$ in that it supplies prescriptions f $_{3}$ : : iecisionmaking and provides explicit measures for assessing the performance of $T T$.

2. However, the proposed methodology requires that the available statistical or time series data be translated into binary numbers before they can be utilized by the method for developing interaction matrices.

3. In terms of criterion 10 , it is clear that neither the three previous approaches nor the proposed methodology supply results that can be readily verified by the user. The user must select monitoring signals, such as to test the 'goodness' of the outputs of the individual method. The previous approaches, however, do not specify explicitly which signals should be monitored. The proposea method, on the other hand, does specify that the outputs of Level 1 should be monitored, since these outputs, in fact, are variables relevant to the 
selected TT. Hence, the user needs to monitor the behavior of only these variables during the planned phases of the selected TT project.

4. In terms of the scope of the efforts needed to use the proposed method in a practical situation, it seems to compare favorably with the three previous appruaches, considering the fact that it utilizes a multidisciplinary structure of TT.

Other results of the comparison, such as the inclusion of group response and the need for computer facilities, are rather obvious and need no further elaboration.

5.1.4 Based on demands of the generating questions (Section $1.3)$ and the understanding of TT provided by the previous studies, the objectives for the proposed IS (Inquiring System) were derived. Two significant objectives are: (a) developing a suitable language for deriving an integrative, unified approach to $T T$, and (b) making the IS useful to planners and policymakers concerned with $\mathrm{TT}$. It is possible to propose a new IS for $T \mathrm{~T}$, using the newer tools of systems analysis, forecasting and policy sciences. A mix of quantitative and qualitative frameworks seems more suitable for the TT problem.

5.1.5 The proposed IS for TT is a three-level hierarchical structure specified in terms of the three critical dimensions of resources required for $T T$, mechanisms to transfer $T T$, and consequences (ox the impact) of TT. 
Level 1 of the proposed IS consists of a morphological tree which explicitly identifies variables and their measures, relevant to TT. A morphological tree, general enough for studying any $\mathrm{T} T$, whether its scope is intercompany interregional or international, was developed (Figure 10). To the author's knowledge, this is the first such tree to be developed for a TT problem. The usefulness of Level 1 for planning and policymaking was discussed.

Level 2 considers interactions between the variables specified in Level 1. Three distinct ways to express the interaction are: binary, continuous and functional. The interaction can be established using five different techniques. Two of these, Ashby's concept of transmission function and Alexander's notion of misfit variables, were discussed.

It was shown that the TT structure can be expressed in terms of the following seveñ matrices: (TxR), (TxM), (TxC), (RxR), (MxM), $(\mathrm{CxC})$ and $(\mathrm{RxC})$. Three of these matrices, $(\mathrm{RxR}),(\mathrm{MxM})$ and $(\mathrm{CxC})$, are self-interaction matrices, while the rest represent crossinteractions between the variables. The self-interactions and crossinteractions can provide indirect relationships between technology and resources, transfer mechanisms and consequences. To account for such indirect paths, a technique to synthesize the seven matrices into three matrices, $\left(T_{x R}\right)_{f},(T \times M)_{f}$ and $(T x C)_{f}$, was suggested and explained. These three matrices are utilized to investigate the performance measures for TT. 
Level 3: of the IS is expressed as a flowgraph which specifies the information, decision and logic requirements to design a most satisfactory TT. The lack of appropriate data and time constraints prevented a computer simulation of Level 3 for this study. Its usefulness, for the present purposes, is to supply a larger framework to develop Levels 1 and 2 .

5.1.6 Three measures were suggested to assess the performance of a TT: feasibility in terms of resources required, efficiency in terms of the applicability of transfer mechanisms and desirability in terms of the consequences of TT. Techniques to estimate nature and magnitude of these performance measures were devised and explained. The inethod for feasibility and efficiency estimation consists in establishing five policy statements that are expressed as assignment or logical statements. The concept of misfit variable is utilized to estimate desirability of TT.

5.1.7 A horizontal TT, specifically the transfer of computer technology (CT) to India, was considered to illustrate the important aspects of the proposed methodology. Levels 1 and 2 were developed. The seven interaction matrices were synthesized to yield (TxR) ${ }_{f}$ $(T \times M)_{f},(T x C)_{f}$ matrices for CT transfer to India. These matrices were used to develop the three performance measures.

5.1.8 The key results obtained from this analysis are: 
(a) CT transfer is feasible in terms of the following resources: tools and equipments and markets for CT. The transfer is infeasible when managerial and entrepreneurial skills are considered. In terms of other resource variables, the transfer is feasible, but under the constraints of cost, productivity and domestic availability.

(b) The most efficient way to transfer CT to India is through the channel of direct investment by business. For hardware and peripheral technology, the channel of licensing arrangement also seems efficient. For software, entrepreneur is a useful mechanism. The channel of direct investment by the Indian government is efficient, only if long transfer times can be tolerated.

(c) The CT transfer is desirable in terms of its impact or: economic variables. However, its impact on some of the sociocultural variables creates misfits. In particular, CT has little desirable impact on such important variables as extent of literacy, quality of medical care, characters of basic social organization and food per capita.

Thus, the CT is desirable in terms of economic variables but its usefulness in improving important sectors, such as literacy, food, basic social organizations and mass communication, is doubtful.

(d) In spite of a rather small impact on some of the sociocultural variables, $\mathrm{CT}$ should be considered an effective technology to borrow, since social, economic, cultural and political environment of 
India seem suitable (in terms of preconditions for take-off) to take advantage of the transfer of CT.

5.1.8 Five recommendations can be made to the Indian planners and policymakers concerning the CT transfer. These are:

(a) Divert resources to overcome the constraints created by a lack of managerial and entrepreneurial skills.

(b) Design means to counter the high cost of capital.

(c) Transfer hardware and peripheral technology through licensing arrangements with appropriate companies and using direct investment by Indian government. Develop software by relying on direct investment by business, domestic or foreign. This arrangement is a compromise between the Indian government's emphasis on public sector investments and a desirability to reduce transfer times.

(d) Intensify efforts to eliminate misfits in sociocultural variables, especially those related to literacy, fored. medical care anci bas id social organization.

(e) Integrate the short-range objectives for CT transfer with the long-range objectives of five-year plans.

5.1.10 The application demonstrates the capability of the proposed IS. It also shows that the concepts can be operationalized for practical purposes, and prescriptions can be provided to planners and policymakers. 
Again, it must be noted that the concepts of the method, rather than the techniques used by the method, are important.

\subsection{Extensions}

Several extensions of the proposed methodology are possible. An obvious one is to employ the concept of 'continuous' interaction in developing the interaction matrices. The methods of fuzzy mathematics seem especially useful in this respect, since the concept of interaction itself is a fuzzy concept. Some other important extensions are: (a) more complete development, implementation and simulation of Level 3, (b) analysis of the sensitivity of the proposed IS in terms of various input conditions, (c) research on yet better structure of IS for $\mathrm{TT}$, (d) determining if equivalent classes of $\mathrm{TT}$ exist and (e) considering explicitly the discounting phenomenon in the TT problems. The phenomenon of discounting can be internalized in the analysis by specifying the elements of the interaction matrices as time-discounted quantities. 
[1] Ackoff, Russell, "Towards a Behavioral Theory of Communication", Management Science, 1957-58, pp.218-34.

[2] Adelman, Irma, and C.T. Morris, Society Politics and Economic Development, Baltimore, The John Hopkins Press, 1967.

[3] Alexander, Christopher, Notes on the Synthesis of Form, Cambridge, Mass., Harvard University Press, 1964, (Fifth printing ---1970).

[4] Allen, Thomas J.,"The Differential Performance of Information Channels In the Transfer of Technology"' In Reference 35, pp.137-154.

[5] Anderson, Norman B., "Data Processing on the Farm", Datamation, March, 1968, pp.84-88.

[6] Ansoff, Igor and Richard G. Brandenburg, "A Language for Organization Design", In Perspective of Planning, Erich Jantsch, Ed., OECD, Paris, 1969, pp.350-375.

[7] Ashby, W. Ross, Introduction to Cybernetics, New York, John Wiley, 1963, Chapters 7,8,9 and 11 .

[8] Ashby, W. Ross, "Analysis of the System to be Modeled", In The Process of Model Building In Behavioral Sciences, R.M. Stogdill, Ed., Ohio State University Press, 1970, pp.94-114. In the Book Trends in General Systems Theory, George Klir, Ed., New York, Jokn Wiley, 1972, pp.78-97.

[10] Ayers, Robert U., Technological Forecasting and Long-range Planning, New York, McGraw Hill 1969.

[11] Bar-Zakay, Samuel N., "Technology Transfer Model", Technological Forecasting and Social Change, Vol. 2, 1971, pp. $32 \mathrm{i}-37$.

[12] Bar-Zakay, Samuel N., "Policyrnaking and Technology Transfer: The Need for National Thinking Laboratories", Policy Sciences, Vol. 2, \#3, Summer 1971, pp.213-227. 
[13] Bauer, P.T., Indian Ecoxomic Policy and Development, New York Praeger Fublishers, 1961 .

[14] Bauer, Raymond E., Second Order Consequences, A Methodological Essay on the Impact of Technology, Cambridge, Mass., The MIT Press, 1969.

[15] Beltran, Sergio F., "Computing in Latin America"', Datamation, March, 1968, pp.93-95.

[16] Bowles, Chester, Ambassador's Report, New York, Harper and Brothers, 1954.

[17] Brooks, Harvey, National Science Policy and Technology Transfer, Conference on Technology Transfer and Innovation, 16-17, May, 1966, Washington, D.C., pp. 53-64.

[18] Butani, D.H., India of the 1970's, New Delhi, Promilla and Co., 1970.

[19] Chaudhari, M.R., Indian Industries: Development and Location, London, Oxford, and IBH Publishing Co., 1970.

[20] Churchman, C. West, The Systems Approach, New York; Dell Publishing Co., 1968 .

[21] Churchman, C. West, The Design of Inquiring Systems, New York, Basic Brooks, 1971 .

[22] Conant, R.C., "Detecting Subsystems of a Complex System", IEEE Transactions on Systems, Man; and Cybernetics, vol. SMC-2, No.4, September 1972, pp.550-553.

[23] Correa, Hector, The Economics of Human Resources, Amsterdam, Holland, North Holland Publishing Co., 1963.

[24] Cyert, Richard M., and J.G. March, A Behavioral Theory of Firm, Englewood Cîíís, N̦.J., Prentice-Hall, 1963.

[25] Denison, Edward F., Why Growth Rates Differ: Postwar Experiences in Nine Western Countries, Washington, D.C., The Brookings Institute, 1967.

[26] Doctors, Samuel I., The Role of Federal Agencies in Technology Transfer, Cambridge, Mass., The MIT Press, 1969.

[27]

- The NASA Technology Transfer Program; An Evaluation of Dissemination System, Ner York, Praeger Publishers, 1971. 
[28] Dror, Yehezkel, Policymaking Reexamined, San Francisco, Chandier, 1968.

[29] Forrester, Jay, Industrial Dynamics, Cambridge, Ivíass., The MIT Press, 1961 .

[30] - Urban Dynamics, Cambridge, Mass., The MIT Press, 1969.

[31] - $\quad$, World Dynamics, Cambridge, Mass ., Wright Allen Press, 1970 .

[32] Gadgil, D.R., Planning and Economic Policy in India, New York, Asia Fublishing House, 1965.

[33] Gordon, T.J., and H. Hayward, Generation of Internally Consistent Scenarios Through the Study of Cross-Impact Matrix, Institute for the Future Report, Miadletown, Conn., 1969.

[34] Government of India, Central Statistical Organization, Statistical Abstracts, India, New Delini, Issued Yearly.

[35] Gruber, W: H. and D.G. Marquis, Eds., Factors in the Transfer of Technology, Cambridge, Mass., The MIT Press, 1969.

[36] Hall, Arthur D.: "Three-Dimensional Morphology of Systems Engineering", IEEE Transactions on Systems, Man and Cybernetics, vol. SSC-5 Number 2, April, 1969, pp.156-160.

[37] Hill, J.B. and J.N. Warfield, "Unified Program Planning", IEEE Transactions on Systems, Man and Cybernetics, vol. SMC -2, \#5, November, 1972, pp.610-621.

[38] Humayun, Kabir, Indian Heritage, New York, Harper and Brothers, 1957.

[39] India Planning Commission, Perspective Planning Division, Notes on Perspective of Development, India: $1960-61$ to $1975-$ 76; Preliminary Draft for Discussion, New Delhi, India, April, 1964.

[40] Industrial Relations Counselors, Inc., Computer Technology Concepts for Management, Papers presented at a symposium conducted by Industrial Relations Counselors, Inc., Published as Industrial Relations Monograph, No. 25, New York, 1965. 
[41] Isenson, Raymond S.: "Project Hindsight: An Empirical Study of the Sources of Ideas Utilized in Operational Weapon Systems," in Reference 35, pp.155-171.

[42] Iyengar, Kesavan N., Fifteen Years of Democratic Planning: Vol. 2, New York, Asia Publishing House, 1965:

[43] Jantsch, Erich, Technological Forecasting in Perspective, Paris, OECD, 1967.

[44] Jantsch, Erich, Technological Planning and Social Futures, London, Associated Business Frogram, Ltd., 1972.

[45] Kasper, Raphael G., Ed., Technology Assessment, Under standing Social Consequences of Technological Applications, New York, Fraeger Publishers, 1972.

[46] Katz, S., Stanley, External Assistance and Economic Growth, New York, Asia Publishing House, 1968.

[47] Kmenta, Jan: "Economic Theory and Transfer of Technology", In Reference 87, pp.39-60.

[48] Koontz, Harold, Ed., T'oward a Unified Theory of Management, New York, McGraw-Hill, 1964.

[49] Krishnamacheri, V.T., Fundamentals of Planning in India, New York, Praeger Publishers, 1962.

[50] Lee, Wayne J., The International Computer Industry, Washington, D.C., Áppilied Library Resources, Inc., 1971.

[51] Lesher, R.I.. and G.J. Horowick, Assessing Technology Transfer, NASA Report SP-5067, 1966.

[52] Lewis, John P., Wanted In India: A Relevant Radicalism, Princeton, N.J., Princeton Univ. Press, Dec. 1969.

[53] Likert, R., "A Technique for the Measurement of Attitudes", Archives of Psychology, Nc. 140, 1932.

[54] Linstone, Harold A., "Four American Futures: Reflections on the Fole of Planning ", Technological Forecasting and Social Change, Vol. 4, \#1, pp.41-60. 
[55] Linstone, Harold A., "On Discounting the Future", Technological Forecasting and Social Change, vol. 4, \#4, pp.335338 .

[56] Linstone, Harold, and M. Turoff, Delphi Method and Its Applications, New York, Elsevier, to be published, Fall 1973.

[57] Mansfield, Edwin, Industrial Research and Technological Innovation, New York, W.W. Norton, 1968 .

[58] Martino, Joseph P., Technological Forecasting for Decisioniviaking = New York, Elsevier, i972.

[59] McClelland, D.C.: The Achieving Society, Princeton, Van Nostrand, N.J., 1961 .

[60] McClelland, D.C., "The Role of Achievement Orientation in the Transfer of Technology", In Reference 35, pp.61-81.

[61] Mesthenne, Emanuel G.: Technological Charge, Its Impact on Society, Harvard Univarsity Press, Cambridge, Massachusetts, 1970.

[62] Michael, Donald N., "On the Social Psychology of Organizational Resistance to Long-Range Social Planning", IEEE Transactions on Systems, Man, and Cybernetics, vol. SMC - 2, No. 5, Nov. 1972, pp.578-584.

[63] Minasian, J.P., "The Economics of Research and Development", In The Rate and Direction of Inventive Activity, Princeton, N.J., Princeton University Press, 1962 .

[64] Mitroff, Ian I. and Murray Turoff: "The Whyg behind the Howg", IEEE Spectrum, March, 1973, pp. 62-71.

[65] Morehouse, Ward, Ed., Science and the Human Condition in India and Pakistan, New Ȳork, Rockefeller Univ. Press, 1968.

[66] National Commission on Technology, Automation and Economic Progress, The Employment Impact of Technological Change, Washington, D.C., 1967 .

[67] OFCD (Organization for Economic Cooperation and Development), Gaps in Technology: Electionic Computers, Paris, ŌECD, 1969. 
[84] Simon, Herbert A., "The Architecture of Complexity", Proc. Amer. Phil. Soc., vol. 106, Dec., 1962, pp.467-482.

[85] Solomon, Martin B., "Economies of Scale and Computer Personnel", Datamation, March, 1970, pp.107-110.

[86] Spencer, Daniel L., "An External Military Presence, Technology Transfer and Structural Change", Kyklos, XVIII, 1965, pp. $451-74$.

[87] Spencer, Daniel L. and Alexander Woroniak, Eds., The Transfer of Technology to Developing Countries, New York, Praegex Press, 1967.

[88] Spencer, Daniel L. and Alexander Woroniak, "The Feasibility of Developing Technology Transfer Functions", Kyklos, XX, $1967, \mathrm{pp} .431-59$.

[89] Spencer, Daniel I., Technology Gap in Perspective, Strategy fox Technology Transfer, Washington, D.C., Spartan Books, 1970 .

[90] - - "Receiving Countries", In the Book EastWest Trade Cap, Stanislow Wasowski, Ed., Praeger Publisher, 1970 .

[91] Taviss, Irene, Ed., The Computer Impact, Englewood Cliffs, N.J., Prentice-Hall, 1970 .

[92] Taylor, Charles Lewis, et al., World Handbock of Political and Social Indicators, Second Edition, New Haven, Conn., Yale Univ. Press, 1972 .

[93] Tel-Aviv University, Computers: 1971-1981, Vol. I. : Report by the Center for Technological and Interdisciplinary Forecasting at Tel Aviv Univ. Aug. 1971.

[94] The Atlantic Institute, The Technology Gap: U.S. and Europe, New York, Praeger Publishers, 1967.

[95] Thompson, James D., Organizations in Action, New York, McGraw-Hill, 1967.

[96] UNESCO: Science and Technology in Asian Development, New York, U.N. Publications, 1970. 
[97] United Nations and UNESCO Bulletin of Statistics, New York, United Nations, Issued monthly and yearly.

[98] U.S. Dept. of Commerce: Global Market Survey, Sept. 1970.

[99] Verghese, B.G., Design for Tomorrow, Bombay, India, The Times of India Press, 1965.

[100] Vickers, Geoffery, Freedom in a Rocking Boat: Changing Values in an Unstable Society, London, The Penguin Press, 1970.

[101] Weiner, Myroxi, The Politics of Scarcity, Public Pressure and Political Response in India, Chicago, The Univ. of Chicago Press, 1967.

[102] Withington, Frederic G., "Trend in MIS Technology", Datamation, Feb. 1970, pp.108-119.

[103] Woodward, Joan, Industrial Organizations: Theory and Practice, Fairlawn, N.J., Oxford University Press, 1965.

[104] Zadeh, Lotfi A., "Outline of a New Approach to the Analysis of Complex Systems", IEEE Transaction on Systems, Man, and Cybernetics, vol. SMiC-3, No. 1, January 1973, ff.28-54.

[105] Zwicky, F., "Morphology of Propulsive Power", Society for Morphological Research, Pasadena, Calif., 1962. 


\section{APPENDIX A}

Some Definitions of Technology and Technology Transfer

This section contains several definitions of technology and technology transfer.

Technology

"Technology denotes the broad area of purposeful applications of the contents of physical, life and behavioral sciences. It comprises the entire notion of technics as well as the medical, agricultural, management and other fields with their total hardware and software contents". (Jantsch)

"Technology... is the systematic application of organized knowledge to practical activities, especially productive ones". (Ayers)

"Technology may be defined as the means or capacity to perform a particular activit $y^{\prime \prime}$. (Gruber and Marquis)

"In the most general sense, the technology refers to knowledge about the performance of certain tasks and/or activities". (Kast and Rosenzweig)

"Technology will mean any tool or technique, any product or proces", any physical equipment or process, any physical equipment or method of doing or making, by which human capability is extended". (Schon)

Technology Transfer

"Technology transfer in its simplest furmulation refers to the purposive movement of established technology or technical innovation from place to place, company to company, or use to use". (Spencer)

"The term technology transfer... will be used in the most common sense, namely, with reference to situations where an established technology in one field finds an unexpected application in some quite different area" = (Ayers) 
- "The transfer of technology must mean the utilization of an existing technique in an instance where it has not previously been used". (Gruber and Marquis)

"Technology transfer can be defined as the process by which the use of technology is diffused throughout society". (Eauer)

"Wherever systematic relational knowledge developed by one group or institution is embodied in a way of doing things by other institutions or groups, we have technology transfer. This can be either transfer from more basic scientific knowledge into technology, or adoptation of an existing technology to a new use". (Brooks) 


\section{APPENDIX B}

A Simple Example to Illustrate the Technique for Synthesizing Interaction Matrices

A simple two-dimensional problem is considered to illustrate the technique outlined in section 3.8.3. Suppose:

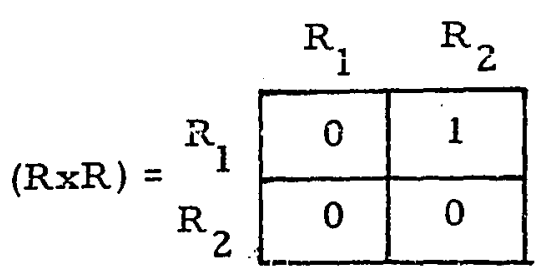

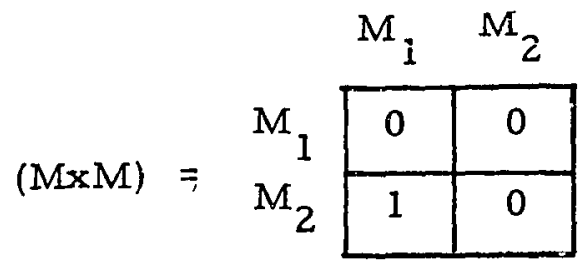

$$
\begin{aligned}
& (\mathrm{CxC})=\begin{array}{c|c|c|}
C_{1} & C_{1} & C_{2} \\
\hline C_{2} & 0 & 0 \\
\hline & 0 & 0 \\
\hline M_{1} & M_{2}
\end{array} \\
& (\mathrm{~T} \times \mathrm{M})=\begin{array}{l|l|l|l|}
\mathrm{T}_{1} & 1 & 0 \\
\cline { 3 - 4 } & \mathrm{T}_{2} & 0 & 1 \\
\hline
\end{array}
\end{aligned}
$$

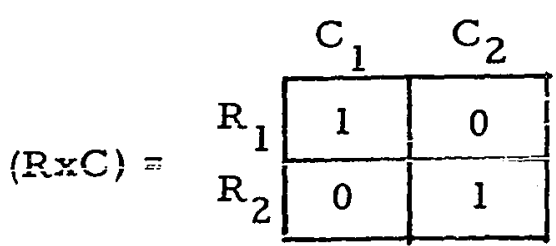

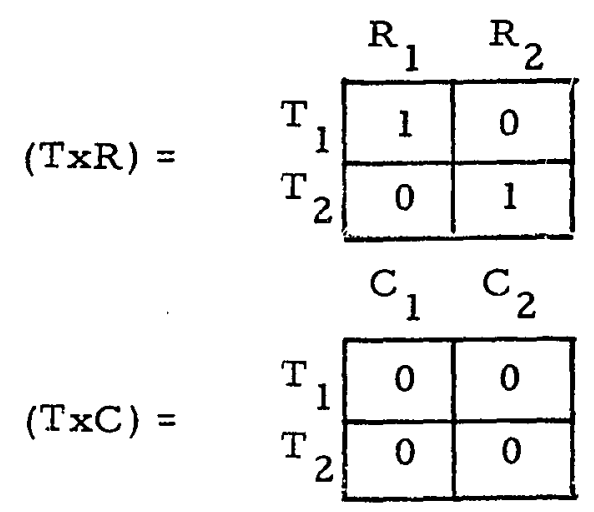

The above matrices represent the following interactions, exhibited in a flowgraph form:

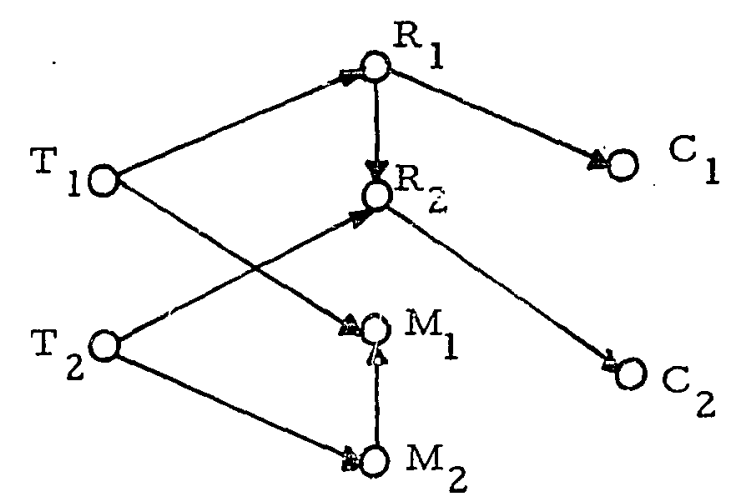


Equation (3.8) yields:

$$
\begin{aligned}
& (T x R)_{s}=[(T x R) \Lambda(R \times R] V(T \times R]
\end{aligned}
$$

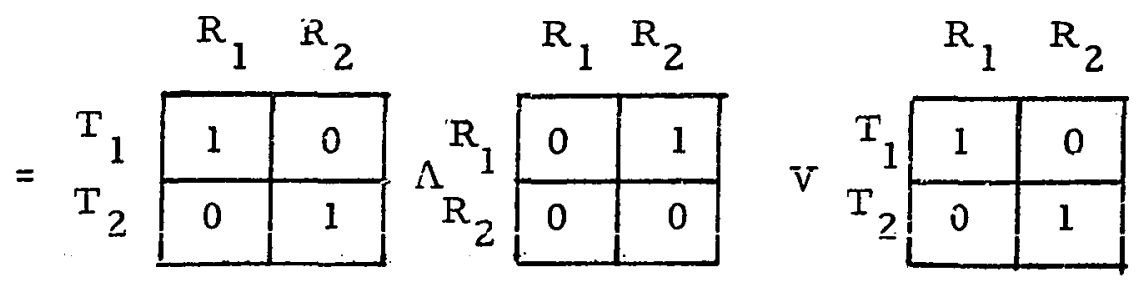

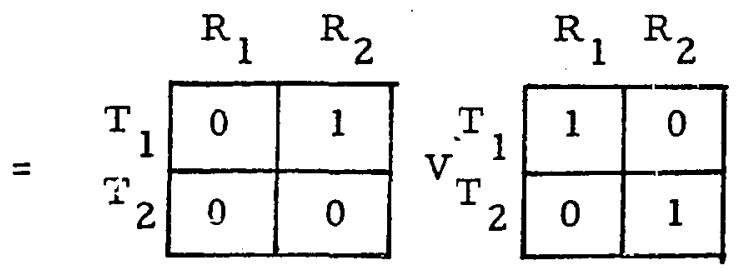

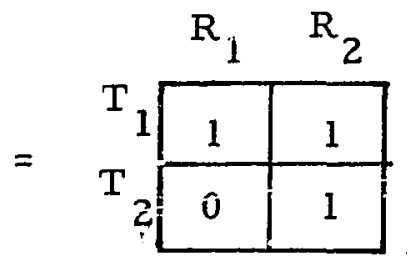

Similarly equations $(3.9),(3.10)$ and $(3.11)$ yield:

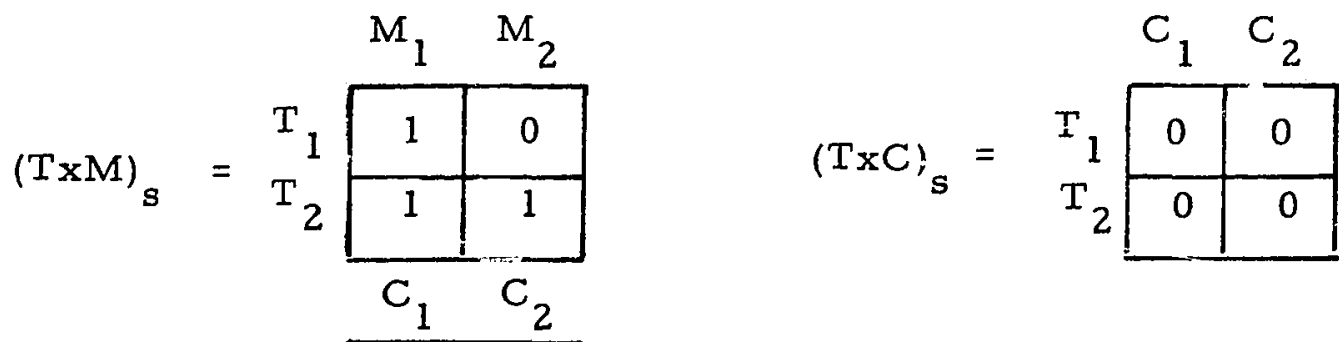

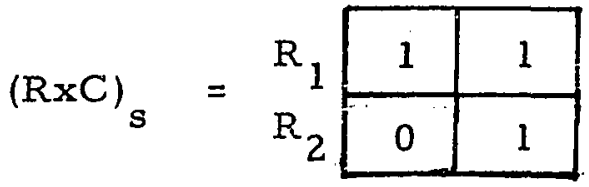

It can be recalled that the matrices with subscript s represent indirect paths created by selif-interaction between the variables. Accounting for indirect paths due to cross-interaction between the variables yields (according to equation 3.12): 


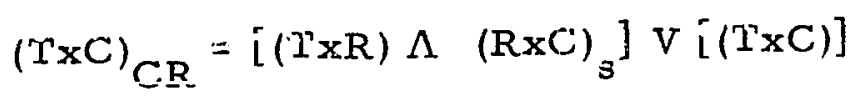
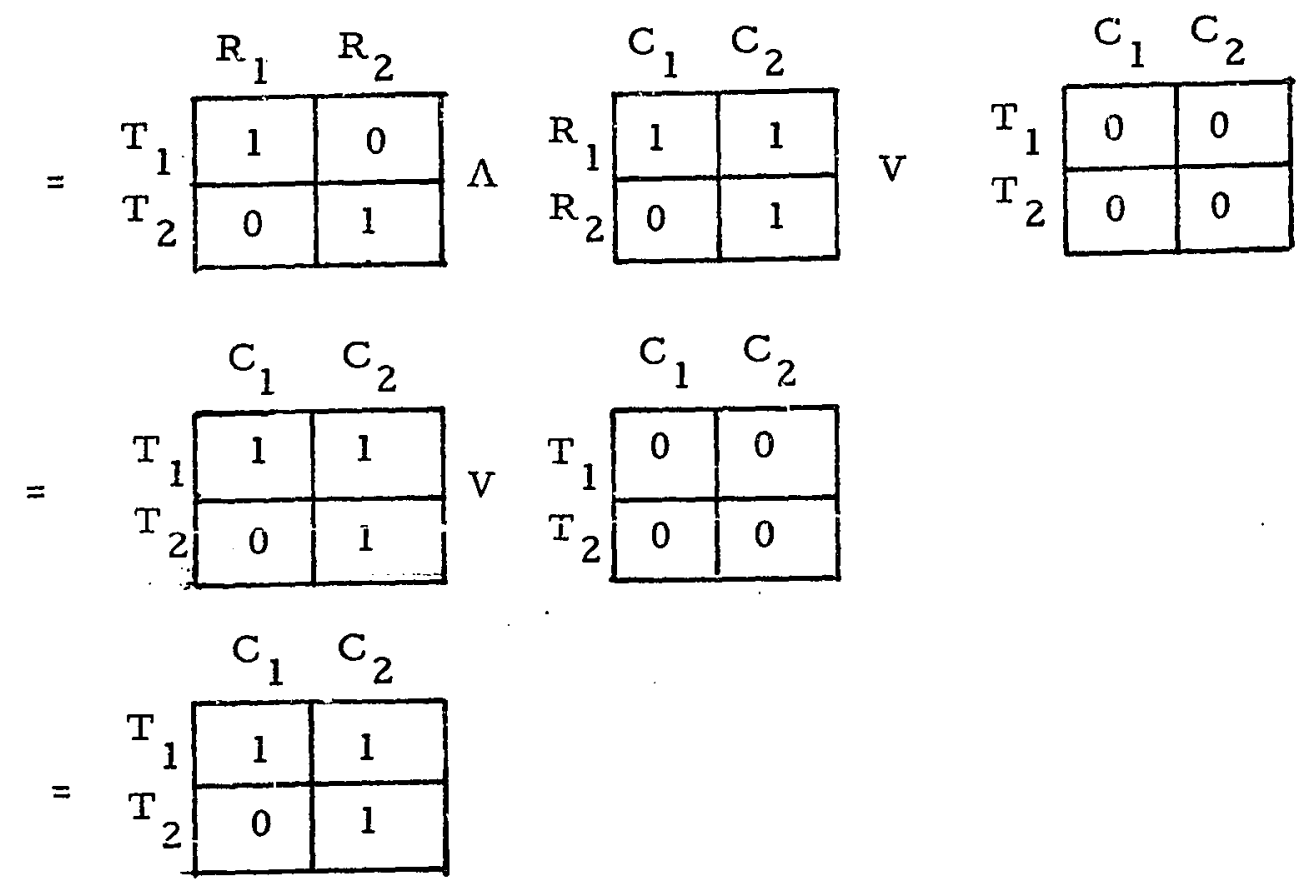

Equations (3.13)-(3.15) yield the final synthesized matrices. Thus:

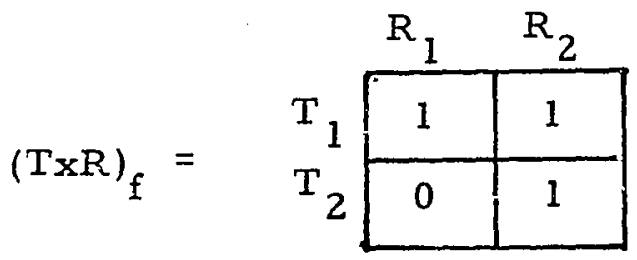

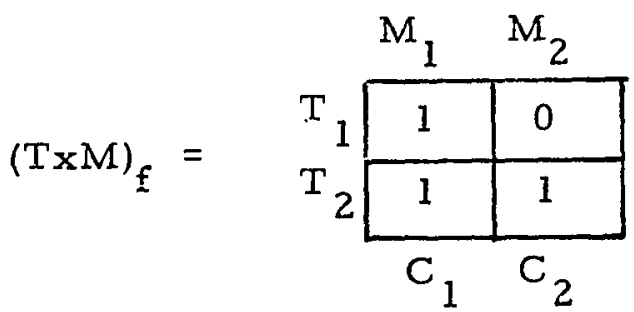

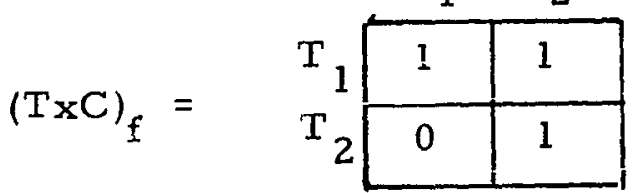

The signal flowgraph for the synthesized matrices is shown below: 


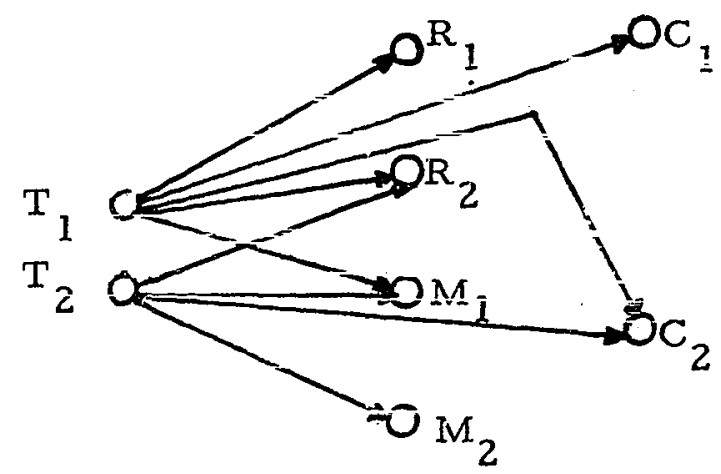

It ghows direct as well as ircirect paths from technoiogy atitributes to resources, transfer mechanisms and consequences. It can be observed that several nonobvious interactions are present. For instance, although (TXC) matrix has all zero elements, there, in fact, are paths leading to consequences from technology. For the systemic treatment of $\mathrm{T} T$ problem, such indirect paths should not be ignored. 